JOURNAL OF THE

AMERICAN MATHEMATICAL SOCIETY

Volume 21, Number 3, July 2008, Pages 615-669

S 0894-0347(08)00591-2

Article electronically published on March 31, 2008

\title{
THE DYNAMICS OF PSEUDOGRAPHS IN CONVEX HAMILTONIAN SYSTEMS
}

\author{
PATRICK BERNARD
}

\section{Contents}

Introduction $\quad 615$

Mather-Fathi theory $\quad 621$

1. Calculus of variations 621

2. Overlapping pseudographs 624

3. Aubry-Mather sets 628

4. Static classes and heteroclinics 637

Abstract mechanisms $\quad 642$

5. The relation and its dynamical consequences $\quad 642$

6. Evolution operators $\quad 645$

7. Coverings 648

8. Mather's mechanism 651

9. Arnold's mechanism for systems with finitely many static classes

Applications $\quad 659$

10. Twist maps 659

11. Generalized Arnold example 659

Appendix $\quad 661$

A. Semi-concave functions 661

B. Uniform families of Hamiltonians 665

Acknowledgements $\quad 668$

References 668

\section{INTRODUCTION}

In this paper, $M$ denotes a connected compact manifold without boundary, of dimension $d$, and $T M$ and $T^{*} M$ are its tangent and cotangent bundles. We shall consider the periodic time-dependent Hamiltonian system generated by a function $H: \mathbb{R} \times T^{*} M \longrightarrow \mathbb{R}$, and denote by $\phi_{s}^{t}$ the flow from time $s$ to time $t$.

Received by the editors October 4, 2004.

2000 Mathematics Subject Classification. Primary 37J40, 37J50.

Key words and phrases. Arnold's diffusion, Mather sets, weak KAM, Hamilton-Jacobi equation.

(C)2008 American Mathematical Society Reverts to public domain 28 years from publication 
(0.1) In order to motivate our discussion, we begin with a precise question: Given two Lagrangian manifolds $\mathcal{G}$ and $\mathcal{G}^{\prime}$ in the cotangent bundle, which are graphs over the base $M$, does there exist a trajectory which connects $\mathcal{G}$ and $\mathcal{G}^{\prime}$, or in other words do there exist times $s<t$ such that the Lagrangian manifold $\phi_{s}^{t}(\mathcal{G})$ intersects $\mathcal{G}^{\prime}$ ?

(0.2) This question formulates some well known problems. As an example, let us suppose that $M=\mathbb{T}^{d}$, and identify the cotangent bundle $T^{*} \mathbb{T}^{d}$ with $\mathbb{T}^{d} \times \mathbb{R}^{d}$. Let us consider the Hamiltonian $H_{0}=h(p)$, where $h: \mathbb{R}^{d} \longrightarrow \mathbb{R}$ is a real function. Such Hamiltonians will be called fully integrable in the sequel. It is known that they leave invariant the tori $\mathbb{T}_{p}:=\mathbb{T}^{d} \times\{p\}$, for $p \in \mathbb{R}^{d}$. As a consequence, the answer to the previous question is obviously negative for $\mathcal{G}=\mathbb{T}_{p}$ and $\mathcal{G}^{\prime}=\mathbb{T}_{p^{\prime}}$, when $p \neq p^{\prime}$. What happens for Hamiltonians $H$ which are close to $H_{0}$ ? For example, it is known that the solar system can be described by a fully integrable Hamiltonian $H_{0}$ if the interactions between planets are neglected. In this example, the variables $p \in \mathbb{R}^{d}$ encode the parameters of the elliptic trajectories of the planets. It is well known that these parameters would not change in time if the interaction between planets did not exist. Understanding for which values of $p$ and $p^{\prime}$ the question $(0.1)$ has a positive answer with $\mathcal{G}=\mathbb{T}_{p}$ and $\mathcal{G}^{\prime}=\mathbb{T}_{p^{\prime}}$, amounts to understanding to what extent the elliptic trajectories will deform under the influence of mutual interactions. In other words, it amounts to understanding the secular dynamics and the stability of the solar system. We will not treat these specific examples in the present papers, although they are parts of our motivation. See [1] and [22] for beautiful and deep examples of perturbations of fully integrable systems.

(0.3) Question $(0.1)$ is especially interesting when the Lagrangian manifolds $\mathcal{G}$ and $\mathcal{G}^{\prime}$ have different Liouville classes (which correspond to the case $p \neq p^{\prime}$ in the discussion above). In this case, we have a problem of non-exact Lagrangian intersection, and it seems that the powerful tools developed to deal with exact intersections provide no interesting insight. In order to study this problem, we make strong assumptions on the Hamiltonian $H$, namely that it is convex, superlinear, periodically time-dependent, and complete; see details in (1.1). We will define an equivalence relation, called a forcing relation and denoted by $\dashv \Vdash$ on the set $H^{1}(M, \mathbb{R})$ of cohomology classes of Lagrangian graphs, in such a way that, if $c \dashv \vdash c^{\prime}$ (we will say that $c$ and $c^{\prime}$ force each over), then the answer to question (0.1) is positive for each Lagrangian graph $\mathcal{G}$ of cohomology $c$ and $\mathcal{G}^{\prime}$ of cohomology $c^{\prime}$. The definition of this equivalence relation is one of the major ideas of the present paper. The key point in considering an equivalence relation is that local information on the equivalence classes can be put together to obtain global information. On the other hand, most of the mechanisms known so far to study questions related to (0.1), the theorem of Birkhoff for twist maps, the geometric construction of Arnold, as well as the variational construction of Mather, can be expressed in this unified setting as local information on the forcing classes (the classes of equivalence of the relation $\dashv$ ). Our main goal in the present paper will be to detail this fact and to study the local properties of the forcing classes.

(0.4) In order to demonstrate the usefulness of our theory, let us provide an example. Proofs and more general statements are given in Section 11. We take $M=\mathbb{T} \times \mathbb{T}^{d-1}$, and denote by $(q, p)=\left(q_{1}, q_{2}, p_{1}, p_{2}\right)$ the points of $T^{*} M$, where 
$q_{1} \in \mathbb{T}, q_{2} \in \mathbb{T}^{d-1}, p_{1} \in \mathbb{R}, p_{2} \in \mathbb{R}^{d-1}$. We consider the time-periodic Hamiltonian

$$
H(t, q, p)=H_{1}\left(t, q_{1}, p_{1}\right)+\left|p_{2}\right|^{2}-V\left(q_{2}\right) F(t, q),
$$

and we assume that the conditions of convexity, super-linearity and completeness are satisfied. In addition, we assume that $F: \mathbb{T} \times \mathbb{T}^{d} \longrightarrow \mathbb{R}$ takes positive values and that $V: \mathbb{T}^{d-1} \longrightarrow \mathbb{R}$ takes positive values except at a single point, say 0 , where its takes the value 0 . The manifold $\mathbb{T} \times \mathbb{R}:=\left\{q_{2}=0, p_{2}=0\right\}$ is then invariant under the Hamiltonian Flow. The restricted flow is generated by the restricted Hamiltonian $H_{1}$. Under these hypotheses, it is not hard to prove (we will do it) that each rotational invariant circle of the restricted dynamics $H_{1}$ admits a homoclinic orbit. We make two additional non-degeneracy assumptions:

(H1) The Hamiltonian $H_{1}$ is generic in the sense that its irrotational invariant circles of rational rotation number are completely periodic. (We allow periodic circles in order to include the case where $H_{1}$ is integrable.)

(H2) We assume a non-degeneracy hypothesis on the set of action minimizing homoclinic orbits to the invariant circles of $H_{1}$. This hypothesis is detailed in Section [1] it should be seen as analogous to the classical hypothesis of transversality of the stable and unstable manifolds in the construction of Arnold. Although in the future we expect to prove that this condition is generic in some sense, we do not discuss any genericity issue here.

Under these hypotheses, our abstract results imply the following.

Theorem. If $P$ and $P^{\prime}$ are given real numbers, there exists a Hamiltonian trajectory $(q(t), p(t))$ and an integer $t \in \mathbb{N}$ such that $p_{1}(0)=P$ and $p_{1}(t)=P^{\prime}$.

(0.5) The systems described in example (0.4) are a priori unstable according to the terminology in use in the world of Arnold's diffusion. This is due to the presence of the distinguished invariant manifold $\left\{q_{2}=p_{2}=0\right\}$, which in many situations is normally hyperbolic. It appears clearly in the fundamental paper of Arnold [1 that the presence of such a hyperbolic invariant manifold intersecting $\mathcal{G}$ and $\mathcal{G}^{\prime}$ greatly favors a positive answer to question (0.1) A priori unstable systems have been widely studied because they appear naturally in the perturbation of completely integrable systems, and are easier to deal with.

In the work of Arnold, it is also assumed that the restriction of the dynamics to the hyperbolic manifold is integrable, say $H_{1}=\left|p_{1}\right|^{2}$ in our example. This means that this invariant manifold is foliated by invariant tori, which he called whiskered tori because of the presence of hyperbolicity. These whiskered tori are the building blocks of Arnold's construction, so that this second hypothesis was very important. The main point in our application is that we do not make this assumption. We only assume that the restricted dynamics are generic, in a clearly specified sense.

In the context of perturbations of fully integrable systems, the restriction of the flow to the hyperbolic manifold is close to integrable, and KAM theory implies the existence of many whiskered tori. However, when precisely computing the various quantities that appear in Arnold's construction, one observes that there does not exist enough tori in general. More precisely, the gap between tori is too big; this is the large gap problem. See for example 21] for a more precise explanation.

Overcoming this problem has long been considered a major challenge. While the classical approaches based on refinements on the scheme of Arnold were worked out in that direction, new variational methods were introduced by John Mather in [24]. It is also worth mentioning the work of Bessi, [7, where the results sketched by 
Arnold are proved using variational methods. This paper contains one on the first relevant achievements of variational methods in these kinds of questions, and it has been very influential. However, these variational methods were facing the same kind of difficulties as classical methods. In several special instances, the Large Gap problem can be bypassed because for specific reasons there exist more whiskered tori. This remark has been exploited to obtain many non-trivial results from Arnold's construction or variational methods. For example, orbits of unbounded speed were built in [8] using the scheme of Arnold. A similar result had previously been obtained by John Mather, 25, using variational methods; see also [19. Other works exploit the same remark in different directions; see for example [6], which elaborates on 7, and many other texts.

Solutions to the Large Gap problem have recently been given by Delshams, de la Llave and Seara (see [13]) and by Treschev (see [29]) using elaborations on Arnold's method. The details in these works are far from simple. Cheng and Yan have also proposed a solution using elaborations on the variational methods initiated by Mather (see [9] and [10]), as well as Z. Xia (see 30] and 31]). Compared to these papers, the spirit of our work is different. We present mechanisms of instability which are more general, but more abstract. We present some examples for illustration and in order to give the reader a hint of how the abstract mechanisms can be used, but we do not try at that point to describe the more general applications. Neither do we discusss the genericity of our hypotheses.

The influence of John Mather's published and unpublished works on the development of variational approaches could not be overestimated. He has announced in [26] very deep results on the perturbation of fully integrable systems in dimension 2 , and given indications on proofs in various talks and lectures. I hope that the tools developed in the present paper will contribute to clarify and extend these results.

(0.6) Let us now enter more precisely into matter. Given a Lipschitz function $u: M \longrightarrow \mathbb{R}$ and a closed smooth form $\eta$ on $M$, we consider the subset $\mathcal{G}_{\eta, u}$ of $T^{*} M$ defined by

$$
\mathcal{G}_{\eta, u}=\left\{\left(x, \eta_{x}+d u_{x}\right), x \in M \text { such that } d u_{x} \text { exists }\right\} .
$$

We call the subset $\mathcal{G} \subset T^{*} M$ an overlapping pseudograph if there exists a closed smooth form $\eta$ and a semi-concave function $u$ such that $\mathcal{G}=\mathcal{G}_{\eta, u}$. See Appendix $\mathrm{A}$ for the definition of semi-concave functions. Each pseudograph $\mathcal{G}$ has a well defined cohomology $c(\mathcal{G}) \in H^{1}(M, \mathbb{R})$ (see $(2.2)$, which is just the De Rham cohomology $[\eta]$ of the closed form appearing in the definition of $\mathcal{G}$. We denote by $\mathbb{P}$ the set of overlapping pseudographs. If $M=\mathbb{T}$ is a circle, then overlapping pseudographs are graphs of functions which have only discontinuities with downward jumps, or in other words functions which can be locally written as the sum of a continuous and a decreasing function. Such sets were introduced in 20, where they are used in very elegant proofs of many known properties of twist maps. In higher dimension, overlapping pseudographs naturally arise from Fathi's approach of Mather theory.

(0.7) We define the forcing relation $\dashv$ on $H^{1}(M, \mathbb{R})$ as follows: We say that $c$ and $c^{\prime}$ force each other (for short $c \dashv c^{\prime}$ ) if there exists an integer $N \in \mathbb{N}$ such that, for each pseudograph $\mathcal{G}$ of cohomology $c$ (resp. $c^{\prime}$ ), there exists a pseudograph $\mathcal{G}^{\prime}$ of 
cohomology $c^{\prime}$ (resp. c) such that

$$
\overline{\mathcal{G}^{\prime}} \subset \bigcup_{1 \leqslant i \leqslant N} \phi_{0}^{i}(\mathcal{G}),
$$

where $\overline{\mathcal{G}^{\prime}}$ is the closure of $\mathcal{G}^{\prime}$ in $T^{*} M$. This definition is certainly one of the most important novelties in the present paper. Note that if $c \dashv \vdash c^{\prime}$, if $\mathcal{G}$ is a Lagrangian graph of cohomology $c$, and if $\mathcal{G}^{\prime}$ is a Lagrangian graph of cohomology $c^{\prime}$, then there exists a Hamiltonian orbit which connects $\mathcal{G}$ and $\mathcal{G}^{\prime}$. As a consequence, understanding the equivalence classes of this relation is a useful tool in the study of our motivating question. Our main goal in the present paper will be to find sufficient conditions for two classes to be equivalent. It turns out that, although the definition seems very strong, the existence of non-trivial forcing classes can be proved in many interesting situations as example (0.4). In fact, many of the known constructions of orbits connecting prescribed regions of phase space (Birkhoff's theory of twist maps, Mather's variational construction of connecting orbits, and Arnold's geometric construction of diffusion) can be adapted to this framework, and rephrased as the existence of large forcing classes.

(0.8) We shall define, following Fathi, an operator $\Phi: \mathbb{P} \longrightarrow \mathbb{P}$ in (2.5) with the following fundamental properties:

$$
\overline{\Phi(\mathcal{G})} \subset \phi(\mathcal{G})
$$

where $\phi:=\phi_{0}^{1}$ is the time-one map of the Hamiltonian flow, and $c(\Phi(\mathcal{G}))=c(\mathcal{G})$. Fathi's weak KAM theorem, [16, states that, for each $c \in H^{1}(M, \mathbb{R})$, the operator $\Phi$ has fixed points of cohomology $c$. We call $\mathbb{V}_{c}$ the set of these fixed points; see Section 3 for details. The fixed points $\mathcal{G}$ satisfy

$$
\overline{\mathcal{G}} \subset \phi(\mathcal{G})
$$

and give rise to compact invariant sets

$$
\tilde{\mathcal{I}}(\mathcal{G}):=\bigcap_{i \in \mathbb{N}} \phi^{-i}(\overline{\mathcal{G}}) .
$$

This provides a new way, due to Albert Fathi, to define various invariant sets previously introduced by Mather in 23] and 24.

(0.9) More precisely, to each cohomology $c \in H^{1}(M, \mathbb{R})$ we associate the nonempty compact invariant sets

$$
\tilde{\mathcal{M}}(c) \subset \tilde{\mathcal{A}}(c) \subset \tilde{\mathcal{N}}(c),
$$

where

$$
\tilde{\mathcal{A}}(c):=\bigcap_{\mathcal{G} \in \mathbb{V}_{c}} \tilde{\mathcal{I}}(\mathcal{G}) \text { and } \tilde{\mathcal{N}}(c):=\bigcup_{\mathcal{G} \in \mathbb{V}_{c}} \tilde{\mathcal{I}}(\mathcal{G}),
$$

are, respectively, called the Aubry set and the Mañé set, and $\tilde{\mathcal{M}}(c)$, called the Mather set, is the union of the supports of the invariant measures of the action of $\phi$ on $\tilde{\mathcal{A}}(c)$ (or equivalently on $\tilde{\mathcal{N}}(c)$ ); see $(3.5)$ for more details. A standing notation will be to denote by $\tilde{\mathcal{X}}$ subsets of $T^{*} M$, and by $\mathcal{X}$ their projection on $M$. 
Beyond answering question (0.1), understanding the forcing relation $\dashv \Vdash$ has many dynamical consequences:

\section{(0.10) Proposition.}

(i) Let $\mathcal{G}$ and $\mathcal{G}^{\prime}$ be two Lagrangian graphs of cohomologies $c$ and $c^{\prime}$. If $c-1 c^{\prime}$, then there exists a time $t \in \mathbb{N}$ such that $\phi_{0}^{t}(\mathcal{G})$ intersects $\mathcal{G}^{\prime}$.

(ii) If $c-1 c^{\prime}$, there exists a heteroclinic trajectory of the Hamiltonian flow between $\tilde{\mathcal{A}}(c)$ and $\tilde{\mathcal{A}}\left(c^{\prime}\right)$.

(iii) Let $c_{i}, i \in \mathbb{Z}$, be a sequence of cohomology classes all of which force the others. Fix for each $i$ a neighborhood $U_{i}$ of $\tilde{\mathcal{M}}\left(c_{i}\right)$ in $T^{*} M$. There exists a trajectory of the Hamiltonian flow which in turn visits all the sets $U_{i}$. In addition, if the sequence stabilizes to $c-$ on the left, or (and) to $c+$ on the right, the trajectory can be assumed negatively asymptotic to $\mathcal{A}(c-)$ or (and) positively asymptotic to $\mathcal{A}(c+)$.

The proof is given in Section 5. Let us now state our main results which, as announced above, describe the local structure of the forcing classes.

(0.11) For each $\mathcal{G} \in \mathbb{V}$, we define the subspace $R(\mathcal{G})$ of $H^{1}(M, \mathbb{R})$ as the set of cohomology classes of smooth closed one-forms whose support is disjoint from $\mathcal{I}(\mathcal{G})$. For each cohomology class $c \in H^{1}(M, \mathbb{R})$, we define the subspace $R(c)$ as

$$
R(c)=\bigcap_{\mathcal{G} \in \mathbb{V}_{c}} R(\mathcal{G}) \subset H^{1}(M, \mathbb{R}) .
$$

The following Theorem reformulates and extends results of John Mather; see [24] and also [3] and [9]. It is proved in Section 8.

Theorem. For each $c_{0} \in H^{1}(M, \mathbb{R})$, there exists a positive $\epsilon$ such that the following holds: Each class $c \in H^{1}(M, \mathbb{R})$ such that $c-c_{0} \in R\left(c_{0}\right)$ and $\left\|c-c_{0}\right\| \leqslant \epsilon$ satisfies $c_{0} \dashv \vdash c$.

In order to illustrate this result, it is useful to consider the case of twist maps $M=\mathbb{T}$. In this case, the reader should check that $R(c)=\mathbb{R}$ or 0 , and that $R(c)=0$ if and only if there exists a rotational invariant circle of cohomology $c$; see (a few) more details in Section 10. The above result then roughly says that rotational invariant circles are the only obstructions to the evolution of action variables, and recovers the theory of Birkhoff. We will explain in Section 11 how this result allows us to overcome the possible absence of invariant circles in example $(0.4)$.

(0.12) There is a natural partition of the Aubry set $\tilde{\mathcal{A}}(c)$ into compact invariant subsets $\tilde{\mathcal{S}}$ called the static classes, see Section 4. A generalized version of the following theorem is proved in Section 9.

Theorem. Assume that there exist only finitely many static classes in $\tilde{\mathcal{A}}(c)$, and that the set $\tilde{\mathcal{N}}(c)-\tilde{\mathcal{A}}(c)$ is not empty and contains finitely many orbits. Then the cohomology $c$ is in the interior of its forcing class.

This result may be seen as a reinterpretation in our langage of the geometric construction of Arnold. We explain in Section [1] how it allows us to take into account the possible presence of invariant circles of $H_{1}$ in example (0.4). We mention that, for a generic Lagrangian, all the Aubry sets $\mathcal{A}(c), c \in H^{1}(M, \mathbb{R})$, have finitely many static classes; see [5]. 
(0.13) Let us now present the content of the paper. The whole paper relies heavily on the notion of a semi-concave function and of equi-semi-concave sets of functions. These notions are presented in Appendix $\mathrm{A}$. In Appendix B. we prove some background results, essentially due to Mather and Fathi, about the properties of the action.

\section{Mather-Fathi theory}

This first part is a survey of the theory of Mather, Mañé and Fathi of globally minimizing orbits, from a point of view very close to the one of Fathi. This survey is presented not only for the convenience of the reader, but also because we need various variations on existing results, and we also need to recast the theory in our framework. In Section 1, we present the context, detail the standing hypotheses, and recall some known results of the calculus of variations which will be of constant use (proofs are given in Appendix B). Pseudographs are defined, and their basic properties studied in Section 2, In Section 3, we use these pseudographs to present Fathi's point of view on Aubry-Mather theory. The theory is continued in Section 4. where we explain Mañé's decomposition in static classes of the Aubry set, and in the construction of homoclinic orbits, due to Fathi [18, Contreras and Paternain [11] (see also [2]), which will play a central role in Section 9].

\section{Abstract mechanism}

This part contains the main novelties of our paper. In Section 5 we define the forcing relation $\dashv$ and explain how various orbits can be built once this relation is understood. We prove Proposition (0.10). We then introduce and study evolution operators on $\mathbb{P}$, which are elaborations around the Lax-Oleinik operator, in Section 6. Section 7 is a parenthesis where we study the action of taking finite Galois covering, which will be essential for applications. The idea of taking finite Galois coverings comes from Fathi [18. In Section 8 we prove and comment Theorem (0.11) In Section 9 we study the case where there exist only finitely many static classes. We generalize and prove Theorem $(0.12)$.

\section{Applications}

In this short part, we detail some straightforward applications of the results obtained before. We hope that it is possible to obtain many more applications by applying our results with Hamiltonian methods such as normal form theory, but this aspect is not discussed here. Section 10 briefly mentions the application to twist maps. Section 11 details $(0.4)$ above.

\section{MATHER-FATHI THEORY}

This part is an overview of the theory of Mather, Mañé and Fathi of globally minimizing orbits, which is oriented towards our future needs. We introduce our main objects. Our point of view is close to the one of Fathi. Most of the material exposed here is a small deformation of results in [23], [15], [27, [12, or [11.

\section{Calculus of variations}

(1.1) We shall consider $C^{2}$ Hamiltonian functions $H: \mathbb{R} \times T^{*} M \longrightarrow \mathbb{R}$. We will denote by $P=(x, p)$ the points of $T^{*} M$. The cotangent bundle is endowed with its standard symplectic structure. We denote by $X(t, P)$ or $X(t, x, p)$ the Hamiltonian vector field of $H$, which is a time-dependent vector field on $T^{*} M$. We fix once and for all a Riemannian metric $g$ on $M$, and use it to define norms of tangent vectors 
and tangent covectors of $M$. We will denote this norm indifferently by $|P|$ or by $|p|$ when $P=(x, p) \in T_{x}^{*} M$. We assume the following standard set of hypotheses:

1. Periodicity. $H(t+1, P)=H(t, P)$ for each $(t, P) \in \mathbb{R} \times T^{*} M$.

2. Completeness. The Hamiltonian vector field $X$ generates a complete flow of diffeomorphisms on $T^{*} M$. We denote by $\phi_{s}^{t}: T^{*} M \longrightarrow T^{*} M$ the flow from time $s$ to time $t$, and by $\phi$ the flow $\phi_{0}^{1}$.

3. Convexity. For each $(t, x) \in \mathbb{R} \times M$, the function $p \longrightarrow H(t, x, p)$ is convex on $T_{x}^{*} M$, with positive definite Hessian. Shortly, $\partial_{p}^{2} H>0$.

4. Superlinearity. For each $(t, x) \in \mathbb{R} \times M$, the function $p \longmapsto H(t, x, p)$ is super-linear, which means that $\lim _{|p| \rightarrow \infty} H(t, x, p) /|p|=\infty$.

(1.2) We associate to the Hamiltonian $H$ a Lagrangian function $L: \mathbb{R} \times T M \longrightarrow \mathbb{R}$ defined by

$$
L(t, x, v)=\sup _{p \in T_{x}^{*} M} p(v)-H(t, x, p) .
$$

The fiberwise differential $\partial_{p} H$ of $H$ can be seen as a mapping

$$
\partial_{p} H: \mathbb{R} \times T^{*} M \longrightarrow \mathbb{R} \times T M
$$

this mapping is a diffeomorphism, whose inverse is given by

$$
\partial_{v} L: \mathbb{R} \times T M \longrightarrow \mathbb{R} \times T^{*} M .
$$

We have the relations $L(t, x, v)=\partial_{v} L(t, x, v)(v)-H\left(t, x, \partial_{v} L(t, x, v)\right)$ and $H(t, x, p)$ $=\partial_{p} H(t, x, p)(p)-L\left(t, x, \partial_{p} H(t, x, p)\right)$. The Lagrangian $L$ satisfies the following properties, which follow from the analogous properties of $H$ :

1. Periodicity. $L(t+1, V)=L(t, V)$ for each $(t, V) \in \mathbb{R} \times T M$.

2. Convexity. For each $(t, x) \in \mathbb{R} \times M$, the function $v \longmapsto L(t, x, v)$ is a convex function on $T_{x} M$, with positive definite Hessian. Shortly, $\partial_{v}^{2} L>0$.

3. Superlinearity. For each $(t, x) \in \mathbb{R} \times M$, the function $v \longmapsto L(t, x, v)$ is super-linear on $T_{x} M$.

See Appendix B for comments related to these hypotheses. The hypotheses listed above are very suitable for using the calculus of variations.

(1.3) Let us fix two times $s>t$ in $\mathbb{R}$ and two points $x$ and $y$ in $M$. Let $\Sigma(t, x ; s, y)$ be the set of absolutely continuous curves $\gamma:[t, s] \longrightarrow M$ such that $\gamma(t)=x$ and $\gamma(s)=y$. As usual, we define the action of the curve $\gamma$ as $A(\gamma)=$ $\int_{t}^{s} L(\sigma, \gamma(\sigma), \dot{\gamma}(\sigma)) d \sigma$. It is known that, for each $C$, the set of curves $\gamma$ in $\Sigma(t, x ; s, y)$ which satisfy $A(\gamma) \leqslant C$ is compact for the topology of uniform convergence. As a consequence, there exist curves minimizing the action. Let us define the value

$$
A(t, x ; s, y)=\min _{\gamma \in \Sigma(t, x ; s, y)} \int_{t}^{s} L(\sigma, \gamma(\sigma), \dot{\gamma}(\sigma)) d \sigma
$$

and let $\Sigma_{m}(t, x ; s, y)$ be the set of curves in $\Sigma$ reaching the above minimum. The set $\Sigma_{m}(t, x ; s, y)$ is not empty, and it is compact for the topology of uniform convergence. Each curve $\gamma(\sigma) \in \Sigma_{m}$ is $C^{2}$ and satisfies the Euler-Lagrange equations. Setting

which is equivalent to

$$
p(\sigma)=\partial_{v} L(\sigma, \gamma(\sigma), \dot{\gamma}(\sigma))
$$

$$
\dot{\gamma}(\sigma)=\partial_{p} H(\sigma, \gamma(\sigma), p(\sigma))
$$


these equations are

$$
\dot{p}(\sigma)=\partial_{x} L(\sigma, \gamma(\sigma), \dot{\gamma}(\sigma))=-\partial_{x} H(\sigma, \gamma(\sigma), p(\sigma)) .
$$

Hence the curve $(\gamma(\sigma), p(\sigma))$ is a trajectory of the Hamiltonian flow.

(1.4) For each minimizing curve $\gamma \in \Sigma_{m}(t, x ; s, y)$, we have

$$
-p(t)=-\partial_{v} L(t, x, \dot{\gamma}(t)) \in \partial_{x}^{+} A(t, x ; s, y),
$$

where $\partial_{x}^{+} A(t, x ; s, y)$ denotes the set of proximal super-differentials of

$$
q \longmapsto A(t, q ; s, y) \text { at point } q=x ;
$$

see Appendix A. We also have

$$
p(s)=\partial_{v} L(s, y, \dot{\gamma}(s)) \in \partial_{y}^{+} A(t, x ; s, y) .
$$

For each $t^{\prime}>t$, the set of functions $(x, y) \longmapsto A(t, x ; s, y), s \geqslant t^{\prime}$ is equi-semiconcave on $M \times M$, hence equi-Lipschitz, see Appendix A. In addition, the three following properties are equivalent:

(i) The set $\Sigma_{m}(t, x ; s, y)$ contains only one point.

(ii) The function $A(t, . ; s, y)$ is differentiable at $x$.

(iii) The function $A(t, x ; s,$.$) is differentiable at y$.

If these equivalent properties hold, and if $\gamma(\sigma)$ is the unique curve of $\Sigma_{m}(t, x ; s, y)$, then setting $p(\sigma)=\partial_{v} L(\sigma, y, \dot{\gamma}(\sigma))$, we have

$$
p(t)=-\partial_{x} A(t, x ; s, y) \text { and } p(s)=\partial_{y} A(t, x ; s, y) .
$$

(1.5) Let $\eta$ be a smooth one-form. We will see the form $\eta$ sometimes as a section of the cotangent bundle $\eta: M \longrightarrow T^{*} M$ and sometimes as a fiberwise linear function on the tangent bundle $\eta: T M \longrightarrow \mathbb{R}$. If the form $\eta$ is closed, then the diffeomorphism $\phi_{\eta}:(x, p) \longmapsto\left(x, p+\eta_{x}\right)$ of $T^{*} M$ is symplectic. The Hamiltonian

$$
H_{\eta}(t, x, p)=H \circ \phi_{\eta}(t, x, p)=H\left(t, x, p+\eta_{x}\right)
$$

satisfies our hypotheses. The associated Lagrangian is $(L-\eta)(t, x, v)=L(t, x, v)-$ $\eta_{x}(v)$, where $\eta$ is considered as a function on $T M$. The following diagram commutes for each $t$ :

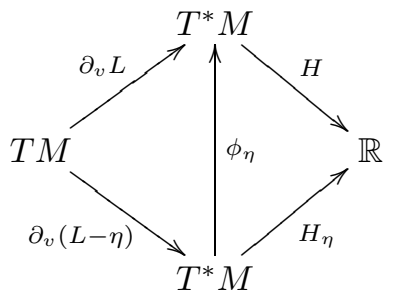

(1.6) We will also consider the modified action

$$
A_{\eta}(t, x ; s, y)=\inf _{\gamma \in \Sigma(t, x ; s, y)} \int_{t}^{s} L(\sigma, \gamma(\sigma), \dot{\gamma}(\sigma))-\eta_{\gamma(\sigma)}(\dot{\gamma}(\sigma)) d \sigma,
$$

which of course satisfies all the properties of (1.4) with the modified expressions

$$
\eta_{x}-p(t) \in \partial_{x}^{+} A_{\eta}(t, x ; s, y) \text { and } p(s)-\eta_{y} \in \partial_{y}^{+} A_{\eta}(t, x ; s, y)
$$

when $(\gamma(\sigma), p(\sigma)):[s, t] \longrightarrow T^{*} M$ is the Hamiltonian trajectory associated to a curve $\gamma \in \Sigma(t, x ; s, y)$ minimizing $A_{\eta}$. 
(1.7) Let $\Omega$ be the set of closed smooth forms on $M$. It is useful to fix once and for all a linear section $S$ of the projection $\Omega \longrightarrow H^{1}(M, \mathbb{R})$. In other words, $S$ is a linear mapping from $H^{1}(M, \mathbb{R})$ to $\Omega$ such that $[S(c)]=c$. We shall abuse notation and denote by $c$ the form $S(c)$ in such a way that the symbol $c$ denotes either a cohomology class or a standard form representing this cohomology class.

(1.8) The following consequence of Appendix B will be useful. See Appendix A for the definition of equi-semi-concave.

Proposition. If $C$ is a bounded subset of $H^{1}(M, \mathbb{R})$ and $\epsilon$ is a positive number, the functions $A_{c}(s, . ; t,),. c \in C, t \geqslant s+\epsilon$, are equi-semi-concave on $M \times M$.

\section{Overlapping pseudographs}

We present the main objects, overlapping pseudographs, and study some basic properties. The relevance of semi-concave functions to this kind of problem was noticed by Albert Fathi.

(2.1) Given a Lipschitz function $u: M \longrightarrow \mathbb{R}$ and a smooth form $\eta$ on $M$, we define the subset $\mathcal{G}_{\eta, u}$ of $T^{*} M$ by

$$
\mathcal{G}_{\eta, u}=\left\{\left(x, \eta_{x}+d u_{x}\right), x \in M, \text { such that } d u_{x} \text { exists }\right\} .
$$

We call the subset $\mathcal{G} \subset T^{*} M$ an overlapping pseudograph if there exists a smooth form $\eta$ and a semi-concave function $u$ such that $\mathcal{G}=\mathcal{G}_{\eta, u}$. See Appendix $\AA$ for the definition of semi-concave functions. We shall denote by $\mathbb{P}$ the set of overlapping pseudographs. Given a pseudograph $\mathcal{G}$ and a subset $U \subset M$, we will denote by $\mathcal{G}_{\mid U}$ the set $\mathcal{G}_{\mid U}:=\mathcal{G} \cap \pi^{-1}(U)$.

(2.2) It is not hard to see that if an overlapping pseudograph $\mathcal{G}$ is represented in two ways as $\mathcal{G}_{\eta, u}$ and $\mathcal{G}_{\mu, v}$, then the closed forms $\eta$ and $\mu$ have the same cohomology in $H^{1}(M, \mathbb{R})$. As a consequence, it is possible to associate to each pseudograph $\mathcal{G}$ a cohomology $c(\mathcal{G})$, in such a way that

$$
c\left(\mathcal{G}_{\eta, u}\right)=[\eta] .
$$

We will denote by $\mathbb{P}_{c}$ the set of overlapping pseudographs of cohomology $c$. If $\mathcal{G}$ is an overlapping pseudograph of cohomology $c$, then $\mathcal{G}$ can be represented in the form $\mathcal{G}=\mathcal{G}_{c, u}$, where $c$ is the standard form defined in (1.7). The function $u$ is then uniquely defined up to an additive constant. As a consequence, denoting by $\mathbb{S}$ the set of semi-concave functions on $M$, and by $\mathbb{P}$ the set of overlapping pseudographs, we have the identification

$$
\mathbb{P}=H^{1}(M, \mathbb{R}) \times \mathbb{S} / \mathbb{R} .
$$

This identification endows $\mathbb{P}$ with the structure of a real vector space. Let us endow the factor $\mathbb{S} / \mathbb{R}$ with the norm $|u|=(\max u-\min u) / 2$, which is the norm induced from the uniform norm on $\mathbb{S}$. More precisely, we have $|u|=\min _{v}\|v\|_{\infty}$, where the minimum is taken on functions $v$ which represent the class $u$. We put on $\mathbb{P}$ the norm

$$
\left\|\mathcal{G}_{c, u}\right\|=|c|+(\max u-\min u) / 2 \leqslant|c|+\|u\|_{\infty} .
$$

The set $\mathbb{P}$ is now a normed vector space. It is also useful to define, for each subset $U \subset M$, the number

$$
\left\|\mathcal{G}_{c, u}\right\|_{U}:=|c|+\left(\sup _{U} u-\inf _{U} u\right) / 2 .
$$


We define in the same way the set $\breve{\mathbb{P}}$ of anti-overlapping pseudographs $\breve{\mathcal{G}}$, which are the sets $\mathcal{G}_{\eta,-u}$, with $\eta$ a smooth form and $u \in \mathbb{S}$. This set is similarly a normed vector space.

(2.3) Lemma. Let $\mathcal{G}$ be an overlapping pseudograph and $\breve{\mathcal{G}}$ be an anti-overlapping pseudograph. If $\mathcal{G}$ and $\breve{\mathcal{G}}$ have the same cohomology, then they have nonempty intersection.

Proof. Let us write $\mathcal{G}=\mathcal{G}_{\eta, u}$ and $\breve{\mathcal{G}}=\mathcal{G}_{\eta,-v}$. Let $x \in M$ be a point minimizing the continuous function $u+v$. Since they are semi-concave, both $u$ and $v$ are differentiable at $x$, and $d u_{x}=-d v_{x}$. It follows that the point $\left(x, \eta_{x}+d u_{x}\right)=$ $\left(x, \eta_{x}-d v_{x}\right)$ belongs both to $\mathcal{G}$ and to $\breve{\mathcal{G}}$.

It is natural to introduce the following definition.

Definition. Let $\mathcal{G}$ be an overlapping pseudograph and $\breve{\mathcal{G}}$ be an anti-overlapping pseudograph. If $\mathcal{G}$ and $\breve{\mathcal{G}}$ have the same cohomology $c$, write them $\mathcal{G}=\mathcal{G}_{c, u}$ and $\breve{\mathcal{G}}=\mathcal{G}_{c, \breve{u}}$. We denote by

$$
\mathcal{G} \wedge \breve{\mathcal{G}} \subset M
$$

the set of points of minimum of the difference $u-\breve{u}$, and by $\mathcal{G} \tilde{\wedge} \breve{\mathcal{G}} \subset \mathcal{G} \cap \breve{\mathcal{G}}$ the set

$$
\mathcal{G} \tilde{\wedge} \breve{\mathcal{G}}:=\mathcal{G} \cap \pi^{-1}(\mathcal{G} \wedge \breve{\mathcal{G}})=\breve{\mathcal{G}} \cap \pi^{-1}(\mathcal{G} \wedge \breve{\mathcal{G}})=\mathcal{G} \cap \breve{\mathcal{G}} \cap \pi^{-1}(\mathcal{G} \wedge \breve{\mathcal{G}}) \subset T^{*} M .
$$

This set is compact, not empty, and is a Lipschitz graph over its projection $\mathcal{G} \wedge \breve{\mathcal{G}}$.

Proof. We have already proved that the set $\mathcal{G} \wedge \breve{\mathcal{G}}$ is not empty. It follows from Appendix (A.8) that both $u$ and $\breve{u}$ are differentiable on $\mathcal{G} \wedge \breve{\mathcal{G}}$, and that the map $x \longmapsto d u_{x}=-d \breve{u}_{x}$ is Lipschitz on this set. This makes the definition meaningful. The set $\mathcal{G} \tilde{\wedge} \breve{\mathcal{G}}$ is compact because it is the image of the compact set $\mathcal{G} \wedge \breve{\mathcal{G}}$ by a Lipschitz map.

(2.4) Let us fix a closed form $\eta$. We define the associated Lax-Oleinik mapping on $C^{0}(M, \mathbb{R})$ by the expression

$$
T_{\eta} u(x)=\min _{q \in M}\left(u(q)+A_{\eta}(0, q ; 1, x)\right) .
$$

Let us recall some important properties of the Lax-Oleinik mapping, which are all direct consequences of the properties of the function $A$ given in (1.4) For each form $\eta$, The functions $T_{\eta} u, u \in C(M, \mathbb{R})$ are equi-semi-concave; see Appendix $\mathrm{A}$. The mapping $T_{\eta}$ is a contraction:

$$
\left\|T_{\eta} u-T_{\eta} v\right\|_{\infty} \leqslant\|u-v\|_{\infty} .
$$

To finish, the mapping $T_{\eta}$ is non-decreasing, and it satisfies $T_{\eta}(a+u)=a+T_{\eta}(u)$ for all real $a$.

(2.5) There exists a unique mapping $\Phi: \mathbb{P} \longrightarrow \mathbb{P}$ such that

$$
\Phi\left(\mathcal{G}_{\eta, u}\right)=\mathcal{G}_{\eta, T_{\eta} u}
$$

for all smooth forms $\eta$ and all semi-concave functions $u$. We have

$$
c(\Phi(\mathcal{G}))=c(\mathcal{G}) .
$$

The mapping $\Phi$ is continuous (see (6.2) for the proof of a more general result). For each cohomology $c$, the image $\Phi\left(\mathbb{P}_{c}\right)$ is a relatively compact subset of $\mathbb{P}_{c}$, as follows directly from the properties of the Lax-Oleinik transformation recalled above. Note 
that this implies the existence of a fixed point of $\Phi$ in each $\mathbb{P}_{c}$; this is how Fathi proved the existence of fixed points. See (3.2) for another proof. We call $\mathbb{V}_{c}$ the set of these fixed points, and $\mathbb{V}=\bigcup_{c} \mathbb{V}_{c}$. We also define the sets

$$
\mathbb{O}:=\bigcap_{n \in \mathbb{N}} \overline{\Phi^{n}(\mathbb{P})}=\bigcap_{n \in \mathbb{N}} \Phi^{n}(\mathbb{P})
$$

and $\mathbb{O}_{c}:=\mathbb{O} \cap \mathbb{P}_{c}$. Note that $\mathbb{O}_{c}$ is compact and invariant under $\Phi$, and that $\mathbb{V} \subset \mathbb{O}$. A pseudograph $\mathcal{G} \in \mathbb{P}_{c}$ belongs to $\mathbb{O}$ if and only if there exists a sequence $\mathcal{G}_{n} \in \mathbb{P}, n \in \mathbb{Z}$, of pseudographs such that $\Phi^{m-n}\left(\mathcal{G}_{n}\right)=\mathcal{G}_{m}$ for all $m \geqslant n$, and such that $\mathcal{G}_{0}=\mathcal{G}$. Note that we then have $\mathcal{G}_{n} \in \mathbb{O}_{c}$ for each $n \in \mathbb{Z}$.

(2.6) The mapping $\Phi$ satisfies

$$
\overline{\Phi(\mathcal{G})} \subset \phi(\mathcal{G}) .
$$

This inclusion is a consequence of the following Proposition, which will be central througrought the paper.

(2.7) Proposition. Let us fix a pseudograph $\mathcal{G}_{\eta, u} \in \mathbb{P}$, an open set $U \subset M$ and two times $s<t$. Let us set

$$
v(x)=\min _{q \in \bar{U}} u(q)+A_{\eta}(s, q ; t, x),
$$

where $\bar{U}$ is the closure of $U$. Let $V \subset M$ be an open set and let $N \subset M$ be the set of points where the minimum is reached in the definition of $v(x)$ for some $x \in V$. If $\bar{N} \subset U$, then

$$
\overline{\mathcal{G}_{\eta, v \mid V}} \subset \phi_{s}^{t}\left(\mathcal{G}_{\eta, u \mid \bar{N}}\right)
$$

and $\mathcal{G}_{\eta, u \mid \bar{N}}$ is a Lipschitz graph above $\bar{N}$. In other words, the function $u$ is differentiable at each point of $\bar{N}$, and the mapping $q \longmapsto d u_{q}$ is Lipschitz on $\bar{N}$.

Addendum. In addition, the Hamiltonian trajectories $(x(\sigma), p(\sigma)):[s, t] \longrightarrow$ $T^{*} M$ which terminate in $\overline{\mathcal{G}_{\eta, v \mid V}}$, i.e. such that $(x(t), p(t)) \in \overline{\mathcal{G}_{\eta, v \mid V}}$ satisfy

$$
\begin{aligned}
v(x(t)) & =u(x(s))+\int_{s}^{t} L(\sigma, x(\sigma), \dot{x}(\sigma))-\eta_{x(\sigma)}(\dot{x}(\sigma)) d \sigma \\
& =u(x(s))+A_{\eta}(s, x(s) ; t, x(t))=\min _{x \in U} u(x)+A_{\eta}(s, x ; t, x(t)) .
\end{aligned}
$$

Proof. Let us fix a point $x \in V$, and consider a point $q \in N$ minimizing in the expression of $v(x)$. Since $q$ is a point of local minimum of the function $u+$ $A_{\eta}(s, ., t, x)$, the semi-concave functions $u$ and $A_{\eta}(s, ., t, x)$ are differentiable at $q$ and satisfy $d u_{q}+\partial_{q} A_{\eta}(s, q, t, x)=0$. In view of (1.4), we have $\partial_{q} A_{\eta}(s, q, t, x)=\eta_{q}-p(s)$, where

$$
(x(\sigma), p(\sigma))=\left(x(\sigma), \partial_{v} L(\sigma, x(\sigma), \dot{x}(\sigma))\right):[s, t] \longrightarrow T^{*} M
$$

is the Hamiltonian trajectory associated to the unique minimizing curve $x(\sigma) \in$ $\Sigma_{m}(s, q, t, x)$. Uniqueness follows from the differentiability of $A_{\eta}(s, ., t, x)$ at $q$; see $(1.4)$. We have

and therefore

$$
(x(s), p(s))=\left(q, d u_{q}+\eta_{q}\right) \subset \mathcal{G}_{\eta, u}
$$

$$
(x(t), p(t))=\phi_{s}^{t}\left(q, d u_{q}+\eta_{q}\right) \in \phi_{s}^{t}\left(\mathcal{G}_{\eta, u \mid N}\right) .
$$

Since the functions $A_{\eta}(s, ., t, x), x \in M$, are equi-semi-concave, they are all $K$-semiconcave for some $K$. It follows that the function $u$ has a $K$-sub-differential at each point of $N$, and therefore at each point of $\bar{N}$. We conclude using (A.7) that the 
function $u$ is differentiable on $\bar{N}$, and that the map $q \longmapsto d u_{q}$ is Lipschitz on $\bar{N}$. As a consequence, we have

$$
\mathcal{G}_{\eta, u \mid \bar{N}}=\overline{\mathcal{G}_{\eta, u \mid N}}
$$

and this set is a Lipshitz graph over $\bar{N}$. Still exploiting (1.4) we get that the function $A_{\eta}(s, q, t,$.$) is differentiable at x$, and satisfies $\partial_{x} A_{\eta}(s, q, t, x)=p(t)-\eta_{x}$. Noticing that the function $v-A_{\eta}(s, q, t,$.$) has a local maximum at x$, we conclude that $d v_{x}=p(t)-\eta_{x}$ if $v$ is differentiable at $x$, and therefore that

$$
\left(x, \eta_{x}+d v_{x}\right)=(x(t), p(t)) \in \phi_{s}^{t}\left(\mathcal{G}_{\eta, u \mid N}\right)
$$

for each point of differentiability $x \in V$ of $v$. In other words, we have the inclusion $\mathcal{G}_{\eta, v \mid V} \subset \phi_{s}^{t}\left(\mathcal{G}_{\eta, u \mid N}\right)$, hence

$$
\overline{\mathcal{G}_{\eta, v \mid V}} \subset \phi_{s}^{t}\left(\overline{\mathcal{G}_{\eta, u \mid N}}\right)=\phi_{s}^{t}\left(\mathcal{G}_{\eta, u \mid \bar{N}}\right) .
$$

(2.8) Let $\mathcal{G}=\mathcal{G}_{c, u}$ be a fixed point of $\Phi$ and let $n<m$ be two relative integers. Following Fathi, we say that a curve $x(t):[n, m] \longrightarrow M$ is calibrated by $\mathcal{G}$ if

$$
u(x(n))+\int_{n}^{m} L(t, x(t), \dot{x}(t))-c_{x(t)}(\dot{x}(t)) d t=T_{c}^{n-m} u(x(m)) .
$$

Note, since $u$ is a fixed point of $\Phi$, that $T_{c}^{n-m} u=u+(m-n) \alpha(c)$, where $\alpha(c)$ is a constant (which, as we will see below depends on $c$ but not on $u$ ). A consequence of the addendum in $(2.7)$ is that the curve $x(t)$ is calibrated by $\mathcal{G}$ if the curve $\left(x(t), p(t)=\partial_{v} L(t, x(\bar{t}), \dot{x}(t))\right)$ is a Hamiltonian trajectory satisfying $(x(m), p(m)) \in$ $\overline{\mathcal{G}}$. Conversely, if $x(t)$ is calibrated by $\mathcal{G}$, then $(x(k), p(k)) \in \mathcal{G}$ for each integer $k \in[n, m[$.

(2.9) The following Corollary is the reason why we have called the elements of $\mathbb{P}$ overlapping.

Corollary. All overlapping pseudographs $\mathcal{G} \in \mathbb{P}$ satisfy $\pi \circ \phi(\mathcal{G})=M$.

Proof. We have $\overline{\Phi(\mathcal{G})} \subset \phi(\mathcal{G})$, and $\pi(\Phi(\mathcal{G}))$ is dense in $M$, so that $\pi(\overline{\Phi(\mathcal{G})})=$ $M$.

(2.10) It is useful, still following Fathi, to define "dual" concepts. We define tihe dual Lax-Oleinik operator associated to a closed form $\eta$ by the expression

$$
\breve{T}_{\eta} u(x)=\max _{q \in M}\left(u(q)-A_{\eta}(0, x ; 1, q)\right), u \in C(M, \mathbb{R}),
$$

and we associate to this operator a mapping $\breve{\Phi}: \breve{\mathbb{P}} \longrightarrow \breve{\mathbb{P}}$ by the expression $\breve{\Phi}\left(\mathcal{G}_{c,-u}\right)=\mathcal{G}_{c, \breve{T}_{c}(-u)} \in \breve{\mathbb{P}}$. We have

$$
\bar{\Phi}(\breve{\mathcal{G}}) \subset \phi^{-1}(\breve{\mathcal{G}})
$$

if $\breve{\mathcal{G}} \in \breve{\mathbb{P}}$. We denote by $\breve{V}$ the set of fixed points of $\breve{\Phi}$. Let $\breve{\mathcal{G}}=\mathcal{G}_{c,-u}$ be a fixed point of $\breve{\Phi}$ and let $n<m$ be two relative integers. We say that a curve $x(t):[n, m] \longrightarrow M$ is calibrated by $\breve{\mathcal{G}}$ if

$$
u(x(m))-\int_{n}^{m} L(t, x(t), \dot{x}(t))-c_{x(t)}(\dot{x}(t)) d t=\breve{T}_{c}^{m-n} u(x(n)) .
$$


(2.11) It is useful to collect a few remarks. We have

$$
\breve{T}_{c} T_{c} u \leqslant u
$$

for all continuous functions $u$. Writing $\breve{T}_{c}^{n+1} T_{c}^{n+1} u=\breve{T}_{c}^{n} \breve{T}_{c} T_{c} T_{c}^{n} u$, we observe that $\breve{T}_{c}^{n} T_{c}^{n} u$ is a non-increasing sequence of functions. Conversely, $T_{c}^{n} \breve{T}_{c}^{n} u$ is nondecreasing for each continuous function $u$.

\section{Aubry-Mather sets}

We use the overlapping pseudographs to recover various invariant sets introduced by Mather, and to study their major properties. We also establish the equivalence between the different definitions of the same sets given in the literature. Most of the section follows Fathi [15, with some minor variations.

(3.1) Proposition. There exists a function $\alpha: H^{1}(M, \mathbb{R}) \longrightarrow \mathbb{R}$ such that, for each continuous function $u$ and each form $\eta$ of cohomology $c$, the sequence $T_{\eta}^{n} u(x)+n \alpha(c), n \geqslant 1$, of continuous functions is equi-bounded and equi-Lipschitz. The function $c \longmapsto \alpha(c)$ is convex and super-linear. More precisely, there exists a constant $K(c)$, which does not depend on the continuous function $u$, such that

$$
\min u-K(c) \leqslant T_{c}^{n} u(x)+n \alpha(c) \leqslant \max u+K(c)
$$

for each $n \in \mathbb{N}$ and $x \in M$.

Proof. Let us fix a cohomology class $c$, and define the sequences

$$
M_{n}(c):=\max _{x \in M} T_{c}^{n}(0)(x) \text { and } m_{n}(c):=\min _{x \in M} T_{c}^{n}(0)(x)
$$

where 0 is the zero function on $M$. Since the functions $T_{c}^{n}(0), n \geqslant 1$, are equi-semiconcave (see Appendix $\mathrm{A}$ ), there exists a constant $K$ such that

$$
0 \leqslant M_{n}(c)-m_{n}(c) \leqslant K
$$

for $n \geqslant 1$. We claim that $M_{n+m}(c) \leqslant M_{n}(c)+M_{m}(c)$. This follows from the inequalities

$$
T_{c}^{m+n}(0)(x)=T_{c}^{m}\left(T_{c}^{n}(0)\right)(x) \leqslant T_{c}^{m}\left(M_{n}(c)\right)(x) \leqslant M_{n}(c)+T_{c}^{m}(0)(x) .
$$

Hence by a classical result on subadditive sequences, we have $\lim M_{n}(c) / n=$ $\inf M_{n}(c) / n$. We denote by $-\alpha(c)$ this limit. In the same way, the sequence $-m_{n}(c)$ is subadditive, hence $m_{n}(c) / n \longrightarrow \sup m_{n}(c) / n$. This limit is also $-\alpha(c)$ because $0 \leqslant M_{n}(c)-m_{n}(c) \leqslant K$. Note that $m_{1}(c) \leqslant-\alpha(c) \leqslant M_{1}(c)$, so that $\alpha(c)$ is indeed a finite number. We have, for all $n \geqslant 1$,

$$
-K-n \alpha(c) \leqslant m_{n}(c) \leqslant-n \alpha(c) \leqslant M_{n}(c) \leqslant K-n \alpha(c) .
$$

Now for all $u \in C(M, \mathbb{R}), n \in \mathbb{N}$ and $x \in M$, we have

$$
\begin{aligned}
\min _{M} u-K & \leqslant \min _{M} u+m_{n}(c)+n \alpha(c) \leqslant T_{c}^{n} u(x)+n \alpha(c) \\
& \leqslant \max _{M} u+M_{n}(c)+n \alpha(c) \leqslant \max _{M} u+K,
\end{aligned}
$$

and we obtain the first conclusion of the Proposition. The explicit definition of the value $m_{n}(c)$ is

$$
m_{n}(c)=\min _{\gamma \in C^{1}([0, n], M)} \int_{0}^{n} L(s, \gamma(s), \dot{\gamma}(s))-c_{\gamma(s)}(\dot{\gamma}(s)) d s .
$$

As a consequence, the function $c \longmapsto m_{n}(c)$ is concave as a minimum of linear functions. Hence each of the functions $c \longmapsto m_{n}(c) / n$ is concave, so that the limit 
$-\alpha(c)$ is concave and the function $\alpha(c)$ is convex. Since $\alpha(c) \geqslant K-m_{1}(c)$, it is enough to prove that $-m_{1}$ is super-linear as a function of $c$ in order to prove that $\alpha$ is also. For each homology class $h \in H_{1}(M, \mathbb{Z})$, let $\gamma_{h}:[0,1] \longrightarrow M$ be a closed curve representing this homology class. We have

$$
-m_{1}(c) \geqslant c(h)-\int_{0}^{1} L\left(s, \gamma_{h}(s), \dot{\gamma}_{h}(s)\right) d s .
$$

This implies that $-m_{1}$, hence $\alpha$, is super-linear. Indeed, in order for a function $f: \mathbb{R}^{n} \longrightarrow \mathbb{R}$ to be super-linear, it is enough that there exists, for each $y \in \mathbb{Z}^{n}$, a value $a_{y}$ such that $f(x) \geqslant y \cdot x-a_{y}$ for each $x$.

(3.2) Proposition. Let us fix a closed form $\eta$ and a continuous function $u$. Let us set

$$
v:=\liminf _{n \longrightarrow \infty}\left(T_{\eta}^{n}(u)+n \alpha([\eta])\right)
$$

then $v$ is a fixed point of $T_{\eta}+\alpha$ and hence $\mathcal{G}_{\eta, v}$ is a fixed point of $\Phi$.

Proof. The one-form $\eta$ is fixed once and for all in this proof, we omit the subscript $\eta$, and denote by $\alpha$ the number $\alpha([\eta])$. Let us first prove that $T v+\alpha \leqslant v$. In order to do so, we fix $x \in M$ and consider an increasing sequence $n_{k}$ of integers such that $T^{n_{k}} u(x)+n_{k} \alpha \longrightarrow v(x)$. Let $q_{k}$ be a point such that $T^{n_{k}} u(x)=T^{n_{k}-1} u\left(q_{k}\right)+$ $A\left(0, q_{k} ; 1, x\right)$ or equivalently, $T^{n_{k}} u(x)+n_{k} \alpha=T^{n_{k}-1} u\left(q_{k}\right)+\left(n_{k}-1\right) \alpha+\alpha+$ $A\left(0, q_{k} ; 1, x\right)$. We can suppose that the sequence $q_{k}$ has a limit $q$. Taking the lim inf in the equality above gives

$$
v(x) \geqslant v(q)+A(0, q, 1, x)+\alpha \geqslant T v(x)+\alpha,
$$

where we have used the equi-continuity of the functions $T^{n} u, n \in \mathbb{N}$.

In order to prove that $T v+\alpha \geqslant v$, just notice that $T^{n} u(x) \leqslant T^{n-1} u(q)+$ $A(0, q ; 1, x)$ for each $q$ and $x$, or equivalently that $T^{n} u(x)+n \alpha \leqslant T^{n-1} u(q)+(n-$ 1) $\alpha+A(0, q ; 1, x)+\alpha$, and take the liminf.

(3.3) Lemma. Let us fix a closed form $\eta$ of cohomology $c$. Let $\mathbb{M} \subset C(M, \mathbb{R})$ be a family of fixed points of the Lax-Oleinik operator $T_{\eta}+\alpha(c)$. Assume that the infimum $v(x)=\inf _{u \in \mathbb{M}} u(x)$ is finite for one (and then each) $x \in M$. Then the function $v$ is a fixed point of $T_{\eta}+\alpha(c)$.

Proof. For all functions $u \in \mathbb{M}$ and all points $x$ and $y$ in $M$, we have $u(x) \leqslant$ $u(y)+A_{\eta}(0, y ; 1, x)+\alpha(c)$. It follows that $v(x) \leqslant v(y)+A_{\eta}(0, y ; 1, x)+\alpha(c)$, so that

$$
v(x) \leqslant \inf _{y}\left(v(y)+A_{\eta}(0, y ; 1, x)\right)+\alpha(c) .
$$

In order to prove the other inequality, let us fix $\epsilon>0$, take a function $u \in \mathbb{M}$ such that $u(x) \leqslant v(x)+\epsilon$, and consider a point $y \in M$ such that $u(x)=u(y)+$ $A_{\eta}(0, y ; 1, x)+\alpha(c)$. We obtain

$v(x) \geqslant u(x)-\epsilon \geqslant u(y)+A_{\eta}(0, y ; 1, x)+\alpha(c)-\epsilon \geqslant v(y)+A_{\eta}(0, y ; 1, x)+\alpha(c)-\epsilon$.

As a consequence, we have $v(x) \geqslant T_{\eta} v(y) \alpha(c)-\epsilon$, and, since this holds for all $\epsilon>0$, the desired inequality follows. 
(3.4) Fixed points of the Lax-Oleinik operator $T_{c}+\alpha(c)$ will be called weak KAM solutions, following Fathi. We denote by $\mathbb{V} \subset \mathbb{P}$ the set of fixed points of $\Phi$, and $\mathbb{V}_{c} \subset \mathbb{P}_{c}$ the set of fixed points of $\Phi$ of cohomology $c$. Sometimes, we will also denote by $\mathbb{V}_{C}$ the set of fixed points of $\Phi$ whose cohomology belongs to the subset $C \subset H^{1}(M, \mathbb{R})$. The set $\mathbb{V}_{c}$ is non-empty for each $c$. If $\mathcal{G} \in \mathbb{V}$, then it follows from (2.6) that

$$
\overline{\mathcal{G}} \subset \phi(\mathcal{G}) .
$$

It is then natural to define the set

$$
\tilde{\mathcal{I}}(\mathcal{G})=\bigcap_{n \in \mathbb{N}} \phi^{-n}(\overline{\mathcal{G}})
$$

which is a nonempty compact $\phi$-invariant subset of $T^{*} M$. We also define

$$
\mathcal{I}(\mathcal{G})=\pi(\tilde{\mathcal{I}}(\mathcal{G})) \subset M .
$$

More generally, for each $\mathcal{G} \in \mathbb{P}$, we define the set

$$
\tilde{\mathcal{I}}(\mathcal{G}):=\bigcap_{n \in \mathbb{N}} \phi^{-n}\left(\overline{\Phi^{n}(\mathcal{G})}\right) .
$$

Since $\phi^{-n}\left(\overline{\Phi^{n}(\mathcal{G})}\right)$ is a non-increasing sequence of compact sets, the set $\tilde{\mathcal{I}}(\mathcal{G})$ is compact and not empty for each $\mathcal{G} \in \mathbb{P}$.

(3.5) For each $\mathcal{G} \in \mathbb{V}$, we define the set $\tilde{\mathcal{M}}(\mathcal{G})$ as the union of the supports of invariant measures of $\phi_{\mid \tilde{\mathcal{I}}(\mathcal{G})}$. If $\mathcal{G} \in \mathbb{V}$ and $\mathcal{G}^{\prime} \in \mathbb{V}$ have the same cohomology $c$, then it is known that

$$
\tilde{\mathcal{M}}(\mathcal{G}) \subset \tilde{\mathcal{I}}\left(\mathcal{G}^{\prime}\right),
$$

hence $\tilde{\mathcal{M}}(\mathcal{G})=\tilde{\mathcal{M}}\left(\mathcal{G}^{\prime}\right)$. As a consequence, the set $\tilde{\mathcal{M}}$, usually called the Mather set, depends only on the cohomology $c$. It will be denoted by

$$
\tilde{\mathcal{M}}(c) \text {, }
$$

and as usual, we will denote by $\mathcal{M}(c)$ the projection $\pi(\tilde{\mathcal{M}}(c))$. We also define the Aubry set in a usual way by

$$
\tilde{\mathcal{A}}(c)=\bigcap_{\mathcal{G} \in \mathbb{V}_{c}} \tilde{\mathcal{I}}(\mathcal{G})
$$

and $\mathcal{A}(c)=\pi(\tilde{\mathcal{A}}(c))$. The Mañé set is defined by

$$
\tilde{\mathcal{N}}(c)=\bigcup_{\mathcal{G} \in \mathbb{V}_{c}} \tilde{\mathcal{I}}(\mathcal{G})
$$

and $\mathcal{N}(c)=\pi(\tilde{\mathcal{N}}(c))$. A bigger set will be useful in some occasions, defined by

$$
\tilde{\mathcal{E}}(c)=\bigcup_{\mathcal{G} \in \mathbb{O}_{c}} \tilde{\mathcal{I}}(\mathcal{G}),
$$

where $\mathbb{O}_{c}$ is as defined in (2.5), As an intersection of Lipschitz graphs, the Aubry set $\tilde{\mathcal{A}}(c)$ is a Lipschitz graph over $\mathcal{A}(c)$. Note however that the Mañé set is not a graph in general. The sets

$$
\tilde{\mathcal{M}}(c) \subset \tilde{\mathcal{A}}(c) \subset \tilde{\mathcal{N}}(c) \subset \tilde{\mathcal{E}}(c)
$$

are compact and invariant under $\phi$. The compactness of $\tilde{\mathcal{N}}(c)$ and $\tilde{\mathcal{E}}(c)$ is mentioned here for completeness; it will be proved later in this section, in (3.12) and (3.13) below. These lemmata also prove that the Mañé set is indeed the set of orbits 
called $c$-minimizing by Mather and semi-static by Mañé, and that the set $\tilde{\mathcal{E}}$ is the set of minimizing orbits called $\tilde{\mathcal{G}}$ in $[3]$.

(3.6) It is possible to associate to each dual fixed point $\breve{\mathcal{G}} \in \breve{\mathbb{V}}$ the invariant set

$$
\tilde{\mathcal{I}}(\breve{\mathcal{G}})=\bigcap_{n \in \mathbb{N}} \phi^{n}(\overline{\mathcal{G}})
$$

and its projection $\mathcal{I}(\breve{\mathcal{G}})$ on $M$. The following is due to Fathi, [17].

Proposition. Let us fix a cohomology $c$, and consider pseudographs $\mathcal{G} \in \mathbb{V}_{c}$ and $\breve{\mathcal{G}} \in \breve{\mathbb{V}}_{c}$. The set $\mathcal{G} \tilde{\wedge} \breve{\mathcal{G}}$ is non-empty, compact and invariant by $\phi$. In addition, this set intersects the Aubry set $\tilde{\mathcal{A}}(c)$ and satisfies

$$
\mathcal{G} \tilde{\wedge} \breve{\mathcal{G}} \subset \tilde{\mathcal{I}}(\mathcal{G}) \cap \tilde{\mathcal{I}}(\breve{\mathcal{G}})
$$

so that

$$
\mathcal{G} \wedge \breve{\mathcal{G}} \subset \mathcal{I}(\mathcal{G}) \cap \mathcal{I}(\breve{\mathcal{G}})
$$

Furthermore, for each pseudograph $\mathcal{G} \in \mathbb{V}_{c}$, there exists a pseudograph $\breve{\mathcal{G}} \in \breve{\mathbb{V}}_{c}$ such that

$$
\mathcal{G} \wedge \breve{\mathcal{G}}=\mathcal{I}(\mathcal{G})=\mathcal{I}(\breve{\mathcal{G}})
$$

In a symmetric way, for each pseudograph $\breve{\mathcal{G}} \in \breve{\mathbb{V}}_{c}$, there exists a pseudograph $\mathcal{G} \in \mathbb{V}_{c}$ such that this relation holds. As a consequence, we have

$$
\tilde{\mathcal{A}}(c)=\bigcap_{\mathcal{G} \in \mathbb{V}_{c}} \tilde{\mathcal{I}}(\mathcal{G})=\bigcap_{\breve{\mathcal{G}} \in \breve{\mathbb{V}}_{c}} \tilde{\mathcal{I}}(\breve{\mathcal{G}})
$$

and

$$
\tilde{\mathcal{N}}(c)=\bigcup_{\mathcal{G} \in \mathbb{V}_{c}} \tilde{\mathcal{I}}(\mathcal{G})=\bigcup_{\breve{\mathcal{G}} \in \breve{V}_{c}} \tilde{\mathcal{I}}(\breve{\mathcal{G}})
$$

Proof. We have already proved that the set $\mathcal{G} \tilde{\wedge} \breve{\mathcal{G}}$ is compact and not empty; see (2.3), Let us prove that it is invariant. In order to do so, we consider a weak KAM solution $u$ and a dual weak KAM solution $\breve{u}$ such that $\mathcal{G}=\mathcal{G}_{c, u}$ and $\breve{\mathcal{G}}=\mathcal{G}_{c, \breve{u}}$. Let $(x(t), p(t)): \mathbb{R} \longrightarrow T^{*} M$ be an orbit of the Hamiltonian flow, such that $(x(0), p(0)) \in \mathcal{G} \tilde{\wedge} \breve{\mathcal{G}}$. Clearly, both $u$ and $\breve{u}$ are differentiable at $x(0)$, and $p(0)=c_{x(0)}+d u_{x(0)}$. For each $m \leqslant n$ in $\mathbb{N}$, we have

$$
\begin{aligned}
u(x(n)) & =\min _{x \in M} u(x)-A_{c}(m, x, n, x(n))+(n-m) \alpha(c) \\
& \leqslant u(x(m))+A_{c}(m, x(m), n, x(n))+(n-m) \alpha(c) .
\end{aligned}
$$

On the other hand, we have $(x(0), p(0)) \in \breve{\mathcal{G}}$; hence, in view of $(2.7)$,

$$
\breve{u}(x(n))=\breve{u}(x(m))+A_{c}(m, x(m), n, x(n))+(n-m) \alpha(c) .
$$

As a consequence, the sequence $n \longmapsto(u-\breve{u})(x(n))$ is non-increasing on $\mathbb{N}$. Since its initial value $(u-\breve{u})(x(0))$ has been chosen to be a minimum of the function $u-\breve{u}$, the sequence must be constant, so that $x(n)$ is a point of $\mathcal{G} \wedge \breve{\mathcal{G}}$ for each $n \geqslant 0$. A symmetric argument shows that this is also true for $n \leqslant 0$. In addition, we obtain that the inequality $u(x(n)) \leqslant u(x(m))+A_{c}(m, x(m), n, x(n))+(n-m) \alpha(c)$ is in fact an equality for $0 \leqslant m \leqslant n$. Since this formula is true in view of $(2.7)$ for $m \leqslant n \leqslant 0$ in $\mathbb{Z}$, we obtain that, for all $m \leqslant n$ in $\mathbb{Z}$,

$$
u(x(n))=u(x(m))+A_{c}(m, x(m), n, x(n))+(n-m) \alpha(c) .
$$


In other words, the curve $x(t)$ is calibrated by $\mathcal{G}$ and by $\breve{\mathcal{G}}$; see $(2.8)$ This implies that $(x(n), p(n)) \in \mathcal{G} \cap \breve{\mathcal{G}}$ for each $n \in \mathbb{Z}$, and, since $x(n) \in \mathcal{G} \wedge \breve{\mathcal{G}}$, we get $(x(n), p(n)) \in \mathcal{G} \tilde{\wedge} \breve{G}$. This proves that $\mathcal{G} \tilde{\wedge} \breve{G}$ is invariant by $\phi$ and contained in $\mathcal{I}(\mathcal{G})$ and in $\mathcal{I}(\breve{\mathcal{G}})$.

Every compact invariant set of $\tilde{\mathcal{I}}(\mathcal{G})$ carries an invariant measure. As a consequence, every compact invariant set of $\tilde{\mathcal{I}}(\mathcal{G})$ intersects the Mather set $\tilde{\mathcal{M}}(c)$; see (3.5). Since $\tilde{\mathcal{M}}(c) \subset \tilde{\mathcal{A}}(c)$, the set $\mathcal{G} \tilde{\wedge} \breve{\mathcal{G}}$, which is a compact and invariant subset of $\mathcal{I}(\mathcal{G})$, intersects $\tilde{\mathcal{A}}(c)$. Let us now fix the pseudograph $\mathcal{G}_{c, u} \in \mathbb{V}_{c}$ and prove the existence of a pseudograph $\breve{\mathcal{G}} \in \breve{\mathbb{V}}_{c}$ such that $\mathcal{G} \wedge \breve{\mathcal{G}}=\mathcal{I}(\mathcal{G})=\mathcal{I}(\breve{\mathcal{G}})$. In order to do so, we set

$$
\breve{u}:=\lim _{n \longrightarrow \infty} \breve{T}_{c}^{n} u-n \alpha(c)=\lim _{n \longrightarrow \infty} \breve{T}_{c}^{n} T_{c}^{n} u .
$$

It follows from (2.11) and (3.1) that the limit exists and that $\breve{u} \leqslant u$. Let $(x(t), p(t))$ : $\mathbb{R} \longrightarrow T^{*} M$ be a Hamiltonian orbit satisfying $(x(0), p(0)) \in \tilde{\mathcal{I}}(\mathcal{G})$. The orbit $x(t)$ is then calibrated by $\mathcal{G}$ (see (2.8) so that the relation

$$
u(x(n))-u(x(m))=A_{c}(m, x(m) ; n, x(n))+(n-m) \alpha(c)
$$

holds for all $m \leqslant n$ in $\mathbb{Z}$. It is clear from this relation that, for each $n \in \mathbb{N}$,

$$
\breve{T}_{c}^{n} u(x(0)) \geqslant u(x(n))-A_{c}(0, x(0) ; n, x(n))=u(x(0))+n \alpha(c),
$$

so that $\breve{T}_{c}^{n} u(x(0))-n \alpha(c)=u(x(0))$; hence $\breve{u}=u$ on $\mathcal{I}(\mathcal{G})$. As a consequence, the set of points minimizing $u-\breve{u}$ contains $\mathcal{I}(\mathcal{G})$. Since we have already proved that this set is contained in $\mathcal{I}(\mathcal{G})$, we can conclude, as desired, that

$$
\mathcal{G}_{c, u} \wedge \mathcal{G}_{c, \breve{u}}=\mathcal{I}(\mathcal{G}) .
$$

Setting $u^{\prime}=\lim T_{c}^{n} \breve{u}+n \alpha(c)=\lim T_{c}^{n} \breve{T}_{c}^{n} \breve{u}$, the same proof shows that

$$
\mathcal{G}_{c, u^{\prime}} \wedge \mathcal{G}_{c, \breve{u}}=\mathcal{I}\left(\mathcal{G}_{c, \breve{u}}\right) .
$$

We claim that $u^{\prime}=u$, so that we have proved

$$
\mathcal{G}_{c, u} \wedge \mathcal{G}_{c, \breve{u}}=\mathcal{I}\left(\mathcal{G}_{c, u}\right)=\mathcal{I}\left(\mathcal{G}_{c, \breve{u}}\right) .
$$

In order to prove that $u^{\prime}=u$, we first recall that $\breve{u} \leqslant u$, so that $T_{c}^{n} \breve{u}+n \alpha(c) \leqslant$ $T_{c}^{n} u+n \alpha(c)=u$, and $u^{\prime} \leqslant u$. On the other hand, for each $\epsilon>0$, there exists $N \in \mathbb{N}$ such that $\breve{T}_{c}^{N} u-N \alpha(c) \leqslant \breve{u}+\epsilon$; hence

$u^{\prime} \geqslant \lim _{n \longrightarrow \infty} T_{c}^{n} \breve{T}_{c}^{N} u+(n-N) \alpha(c)-\epsilon \geqslant \lim _{n \longrightarrow \infty} T_{c}^{n-N} u+(n-N) \alpha(c)-\epsilon=u-\epsilon$.

We have proved that $u^{\prime}=u$.

The pairs $(u, \breve{u})$ of fixed points of $T_{c}+\alpha(c)$ and $\breve{T}_{c}-\alpha(c)$ which satisfy

$$
\breve{u}=\lim \breve{T}_{c}^{n} u-n \alpha(c) ; u=\lim T_{c}^{n} \breve{u}+n \alpha(c)
$$

are conjugate in the sense of Fathi.

(3.7) Proposition. The restriction to $\mathbb{V}$ of the function $c: \mathbb{P} \longrightarrow H^{1}(M, \mathbb{R})$ is continuous and proper.

Proof. Let us consider a compact subset $C$ of $H^{1}(M, \mathbb{R})$. The family of Hamiltonians $H\left(t, x, c_{x}+p\right), c \in C$, is a uniform family of Hamiltonians; see Appendix $\mathrm{B}$. As a consequence, the associated functions $A_{c}(0, . ; 1,),. c \in C$, form an equi-semi-concave family of functions on $M \times M$. As a consequence, the functions $A_{c}(0, x ; 1,),. c \in$ $C, x \in M$, form an equi-semi-concave family of functions on $M$; see Appendix A 
It follows that the functions $u(x)+A_{c}(0, x ; 1,),. c \in C, x \in M$, also form an equisemi-concave family; hence the functions $\min _{x} u(x)+A_{c}(0, x ; 1,),. c \in C$, form an equi-semi-concave family. As a consequence, the set $\Phi\left(\mathbb{P}_{C}\right)$ is relatively compact. Since the set $\mathbb{V}_{C}$ is obviously closed and contained in $\Phi\left(\mathbb{P}_{C}\right)$, it is compact.

We have proved the following Lemma, which is interesting in itself:

Lemma. If $C$ is a compact subset of $H^{1}(M, \mathbb{R})$, the set $\Phi\left(\mathbb{P}_{C}\right)$ is equi-semi-concave.

(3.8) Following Mather, we will use the function

$$
h_{c}(x, y):=\liminf _{n \longrightarrow \infty} A_{c}^{n}(x, y)+n \alpha(c) .
$$

In view of $(3.2)$, the function $h_{c}(x,$.$) is a fixed point of T_{c}+\alpha(c)$. Similarly, the function $-h_{c}(., y)$ is a fixed point of $\breve{T}_{c}-\alpha(c)$. Let us recall some basic properties of the function $h_{c}$ :

- For each $x, y, z \in M$ and $c \in H^{1}(M, \mathbb{R})$, we have the triangle inequality $h_{c}(x, y)+h_{c}(y, z) \geqslant h_{c}(x, z)$.

- For each $x, y \in M$ and $c \in H^{1}(M, \mathbb{R})$, we have $h_{c}(x, y)+h_{c}(y, x) \geqslant h_{c}(x, x) \geqslant$ 0 .

- For each compact set $C \subset H^{1}(M, \mathbb{R})$, the set of functions $h_{c}: M \times M \longrightarrow$ $\mathbb{R}, c \in C$, is equi-semi-concave.

(3.9) Proposition. If the pseudograph $\mathcal{G}_{c, u}$ is a fixed point of $\Phi$, then we have

$$
u(y)-u(x) \leqslant h_{c}(x, y)
$$

for each $x$ and $y$. In addition,

$$
u(x)=\min _{y \in M} u(y)+h_{c}(y, x)=\min _{a \in \mathcal{A}(c)} u(a)+h_{c}(a, x) .
$$

Proof. We have, for each $n, u=T_{c}^{n} u+n \alpha(c)$. As a consequence, for each $n$,

$$
u(x)=\min _{y \in M}\left(u(y)+A_{c}(0, y ; n, x)+n \alpha(c)\right) .
$$

We obtain the inequality $u(x) \leqslant u(y)+A_{c}(0, y ; n, x)+n \alpha(c)$ and, by taking the liminf, $u(x) \leqslant u(y)+h_{c}(y, x)$. In order to obtain the first equality, we consider a point $y_{n} \in M$ such that

$$
u(x)=u\left(y_{n}\right)+A_{c}\left(0, y_{n} ; n, x\right)+n \alpha(c) .
$$

We consider an increasing sequence $n_{k}$ of integers such that the subsequence $y_{n_{k}}$ has a limit $y$, and refine this subsequence in such a way that the subsequence $A_{c}\left(0, y, n_{k}, x\right)$ has a limit $l$. We have

$$
u(x)=u(y)+l \geqslant u(y)+h_{c}(y, x) .
$$

Cumulated with the previously shown inequality, this proves the first equality in the statement. In order to prove the second equality, notice that the set of points $y$ which minimize the function $u()+.h_{c}(., x)$ is precisely the set $\mathcal{G} \wedge \mathcal{G}_{c,-h_{c}(., x)}$, and that $\mathcal{G}_{c,-h_{c}(., x)} \in \breve{\mathbb{V}}_{c}$. By $(3.6)$ the set $\mathcal{G} \wedge \mathcal{G}_{c,-h_{c}(., x)}$ intersects $\mathcal{A}(c)$.

(3.10) Corollary. For each $x$ and $y$ in $M$ and $c \in H^{1}(M, \mathbb{R})$, we have

$$
h_{c}(x, y)=\min _{z \in M} h_{c}(x, z)+h_{c}(z, y)=\min _{a \in \mathcal{A}(c)} h_{c}(x, a)+h_{c}(a, y) .
$$

The following result connects our definition of the Aubry set to the one of Mather. 
(3.11) Proposition. The Aubry set $\mathcal{A}(c)$ is the set of points $x$ such that $h_{c}(x, x)=$ 0 .

Proof. Let us consider a Hamiltonian trajectory $(x(t), p(t)): \mathbb{R} \longrightarrow T^{*} M$ such that $(x(0), p(0)) \in \tilde{\mathcal{A}}(c)$. This trajectory is calibrated by each fixed point of $T_{c}+$ $\alpha(c)$, and so in particular by $h_{c}(x(0),$.$) . Consequently, we have$

$$
h_{c}(x(0), x(n))-h_{c}(x(0), x(0))=A_{c}(0, x(0) ; n, x(n))+n \alpha(c) .
$$

Taking a subsequence such that $x(n)$ has a limit $x$, and then a subsequence such that $A_{c}(0, x(0) ; n, x)+n \alpha(c)$ is converging to a limit $l \geqslant h_{c}(x(0), x)$, we get, at the limit,

$$
h_{c}(x(0), x)-h_{c}(x(0), x(0)) \geqslant h_{c}(x(0), x)
$$

thus $h_{c}(x(0), x(0)) \leqslant 0$ and then $h_{c}(x(0), x(0))=0$. We have proved that the function $h_{c}(x, x)$ vanishes on $\mathcal{A}(c)$.

Conversely, let us assume that $h_{c}(x, x)=0$. Then there exists an increasing sequence $n_{k}$ of integers and a sequence of trajectories $\left(x_{k}(t), p_{k}(t)\right):\left[0, n_{k}\right] \longrightarrow$ $T^{*} M$ such that $x(0)=x\left(n_{k}\right)=x$ and

$$
\int_{0}^{n_{k}} L\left(t, x_{k}(t), \dot{x}_{k}(t)\right)-c_{x_{k}(t)}\left(\dot{x}_{k}(t)\right)+\alpha(c) d t=A_{c}\left(0, x ; n_{k}, x\right)+n_{k} \alpha(c) \longrightarrow 0 .
$$

Let $y_{k}:\left[-n_{k}, n_{k}\right] \longrightarrow M$ be the curve such that $y_{k}(t)=x_{k}\left(t+n_{k}\right)$ for $-n_{k} \leqslant t \leqslant 0$ and $y_{k}(t)=x_{k}(t)$ for $t \geqslant 0$. The sequence $y_{k}$ is $C^{2}$-bounded. Hence, by taking a subsequence, we can suppose that $y_{k}$ is converging with its derivative, uniformly on compact sets, to a limit $y(t): \mathbb{R} \longrightarrow M$. We claim that this limit $y$ is calibrated by each fixed point of $T_{c}+\alpha(c)$. Let $u$ be a such a fixed point. We have, for each $n \in \mathbb{N}$ and $k$ large enough,

$$
\begin{aligned}
0 \geqslant & u\left(y_{k}(n)\right)-u\left(y_{k}(-n)\right)-A_{c}\left(-n, y_{k}(-n) ; n, y_{k}(n)\right)-2 n \alpha(c) \\
= & u\left(y_{k}\left(n_{k}\right)\right)-u\left(y_{k}\left(-n_{k}\right)\right)-A_{c}\left(-n_{k}, y_{k}\left(-n_{k}\right) ; n_{k}, y_{k}(n)\right)-2 n_{k} \alpha(c) \\
& -\left(u\left(y_{k}\left(n_{k}\right)\right)-u\left(y_{k}(n)\right)-A_{c}\left(n, y_{k}(n) ; n_{k}, y_{k}(n)\right)-\left(n_{k}-n\right) \alpha(c)\right) \\
& -\left(u\left(y_{k}(-n)\right)-u\left(y_{k}\left(-n_{k}\right)\right)-A_{c}\left(-n_{k}, y_{k}\left(-n_{k}\right) ;-n, y_{k}(-n)\right)-\left(n_{k}-n\right) \alpha(c)\right) \\
\geqslant & u\left(y_{k}\left(n_{k}\right)\right)-u\left(y_{k}\left(-n_{k}\right)\right)-A_{c}\left(-n_{k}, y_{k}\left(-n_{k}\right) ; n_{k}, y_{k}(n)\right)-2 n_{k} \alpha(c) \\
= & -A_{c}\left(-n_{k}, x ; n_{k}, x\right)-2 n_{k} \alpha(c)=-2 A_{c}\left(0, x ; n_{k}, x\right)-2 n_{k} \alpha(c) .
\end{aligned}
$$

For fixed $n$, we now take the limit $k \longrightarrow \infty$, and get that

$$
u(y(n))-u(y(-n))=A_{c}(-n, y(-n) ; n, y(n))+2 n \alpha(c) .
$$

Consequently, the curve $y(t)$ is calibrated by $u$. Since this holds for each weak KAM solution $u$, we have $x=y(0) \in \mathcal{A}(c)$.

The following well-known result connects our definition of the Mañé set with the usual one, and implies its compactness.

(3.12) Lemma. The following properties are equivalent for a continuous curve $P(t)=(x(t), p(t)): \mathbb{R} \longrightarrow T^{*} M:$

(i) The curve $P(t)$ is a Hamiltonian trajectory and $P(\mathbb{Z}) \subset \tilde{\mathcal{N}}(c)$.

(ii) The curve $P(t)$ satisfies $p(t)=\partial_{v} L(t, x(t), \dot{x}(t))$, and there exists $\mathcal{G}_{c, u} \in \mathbb{V}_{c}$ such that, for each $m \geqslant n$ in $\mathbb{Z}$, we have

$$
u(x(m))-u(x(n))=\int_{n}^{m} L(t, x(t), \dot{x}(t))-c_{x(t)}(\dot{x}(t)) d t+(m-n) \alpha(c) .
$$


(iii) The curve $P(t)$ satisfies $p(t)=\partial_{v} L(t, x(t), \dot{x}(t))$, and for each $m \geqslant n$ in $\mathbb{Z}$, we have

$\int_{n}^{m} L(t, x(t), \dot{x}(t))-c_{x(t)}(\dot{x}(t)) d t+(m-n) \alpha(c)=\min _{l \in \mathbb{N}, l>0} A_{c}(0, x(n) ; l, x(m))+l \alpha(c)$.

Proof. We shall prove that $(i i i) \Rightarrow(i i)$. The other implications are left to the reader. Let $P(t)$ be a curve satisfying (iii) and let $n_{k}$ be an increasing sequence of integers such that $x\left(-n_{k}\right)$ has a limit $\alpha$. Then we have, for $m \geqslant n$,

$$
\begin{array}{rl}
\int_{n}^{m} & L(t, x(t), \dot{x}(t))-c_{x(t)}(\dot{x}(t)) d t+(m-n) \alpha(c) \\
= & \int_{-n_{k}}^{m} L(t, x(t), \dot{x}(t))-c_{x(t)}(\dot{x}(t))+\alpha(c) d t \\
& -\int_{-n_{k}}^{n} L(t, x(t), \dot{x}(t))-c_{x(t)}(\dot{x}(t))+\alpha(c) d t \\
= & A_{c}\left(-n_{k}, x\left(-n_{k}\right) ; m, x(m)\right)+\left(m+n_{k}\right) \alpha(c) \\
& -A_{c}\left(-n_{k}, x\left(-n_{k}\right) ; n, x(n)\right)+\left(n+n_{k}\right) \alpha(c) .
\end{array}
$$

By $(i i i)$, we have

$$
\begin{aligned}
& A_{c}\left(-n_{k}, x\left(-n_{k}\right) ; m, x(m)\right)+\left(m+n_{k}\right) \alpha(c)=\min _{l \in \mathbb{N}, l>0} A_{c}\left(0, x\left(-n_{k}\right) ; l, x(m)\right)+l \alpha(c) \\
& \quad \leqslant h_{c}\left(x\left(-n_{k}\right), x(m)\right)
\end{aligned}
$$

which implies that

$$
A_{c}\left(-n_{k}, x\left(-n_{k}\right) ; m, x(m)\right)+\left(m+n_{k}\right) \alpha(c) \longrightarrow h_{c}(\alpha, x(m))
$$

as $k \longrightarrow \infty$. Similarly,

$$
A_{c}\left(-n_{k}, x\left(-n_{k}\right) ; n, x(n)\right)+\left(n+n_{k}\right) \alpha(c) \longrightarrow h_{c}(\alpha, x(n)),
$$

so that

$$
h_{c}(\alpha, x(m))-h_{c}(\alpha, x(n))=\int_{n}^{m} L(t, x(t), \dot{x}(t))-c_{x(t)}(\dot{x}(t)) d t+(m-n) \alpha(c) .
$$

We have proved $(i i)$ with $u=h_{c}(\alpha,$.$) .$

We now give equivalent definitions for the set $\tilde{\mathcal{E}}$. The following Lemma shows that the set $\tilde{\mathcal{E}}$ is the set called $\tilde{\mathcal{G}}$ in $[3$, and implies its compactness.

(3.13) Lemma. The following properties are equivalent for a continuous curve $P(t)=(x(t), p(t)): \mathbb{R} \longrightarrow T^{*} M:$

(i) The curve $P(t)$ is a Hamiltonian trajectory and $P(\mathbb{Z}) \subset \tilde{\mathcal{E}}(c)$.

(ii) The curve $P(t)$ satisfies $p(t)=\partial_{v} L(t, x(t), \dot{x}(t))$, and there exists a sequence $u_{n}$ of functions such that, for each $m \geqslant n$, we have $T_{c}^{m-n} u_{n}=u_{m}$ and

$$
u_{m}(x(m))-u_{n}(x(n))=\int_{n}^{m} L(t, x(t), \dot{x}(t))-c_{x(t)}(\dot{x}(t)) d t .
$$

(iii) The curve $P(t)$ satisfies $p(t)=\partial_{v} L(t, x(t), \dot{x}(t))$, and for each $m \geqslant n$, we have

$$
\int_{n}^{m} L(t, x(t), \dot{x}(t))-c_{x(t)}(\dot{x}(t)) d t=A_{c}(n, x(n) ; m, x(m)) .
$$


Proof. $(i i) \Rightarrow(i)$. Then for each pair $m \geqslant n$ of integers, the curve $x(t):[n, m] \longrightarrow$ $M$ is minimizing the action between its endpoints. Hence the curve $P(t)$ is a Hamiltonian trajectory. It follows from $(2.7)$ that, for each $n \geqslant 0$, we have $P(n) \in$ $\overline{\mathcal{G}_{c, u_{n}}}$, and since $P(n)=\phi^{n}(P(0))$, we have

$$
P(0) \in \phi^{-n}\left(\overline{\left.\Phi^{n}\left(\mathcal{G}_{c, u_{0}}\right)\right)} .\right.
$$

This inclusion holds for all $n$, so that $P(0) \in \tilde{\mathcal{I}}\left(\mathcal{G}_{c, u_{0}}\right)$. Now $(i)$ follows from the fact that $\mathcal{G}_{c, u_{0}} \in \mathbb{O}$ and that $\tilde{\mathcal{I}}\left(\mathcal{G}_{c, u_{0}}\right)$ is invariant under $\phi$.

$(i) \Rightarrow(i i)$. There exists a pseudograph $\mathcal{G}_{c, u_{0}} \in \mathbb{O}$ such that $P(0) \in \tilde{\mathcal{I}}\left(\mathcal{G}_{c, u_{0}}\right)$. There exists a sequence $u_{n}, n \in \mathbb{Z}$, of functions on $M$ such that $T_{c}^{m-n} u_{n}=u_{m}$ for $m \geqslant n$. For each $m \geqslant 0$, since $P(m) \in \overline{\mathcal{G}_{c, u_{m}}}$, we have

$$
u_{m}(x(m))-u_{0}(x(0))=\int_{0}^{m} L(t, x(t), \dot{x}(t))-c_{x(t)}(\dot{x}(t)) d t .
$$

On the other hand, for each $n \leqslant 0$, we can find by minimization a curve $y_{n}(t)$ : $[n, 0] \longrightarrow M$ such that $y_{n}(0)=x(0)$ and

$$
u_{0}\left(y_{n}(0)\right)-u_{n}\left(y_{n}(n)\right)=\int_{n}^{0} L\left(t, y_{n}(t), \dot{y}_{n}(t)\right)-c_{y_{n}(t)}\left(\dot{y}_{n}(t)\right) d t .
$$

There exists a subsequence $n_{k}$ such that the curves $y_{n_{k}}(t)$ converge, uniformly on compact sets, to a limit $y(t):(-\infty, 0] \longrightarrow M$. By $(1.3)$, this curve satisfies, for all $n \leqslant 0$,

$$
u_{0}(y(0))-u_{n}(y(n))=\int_{n}^{0} L(t, y(t), \dot{y}(t))-c_{y(t)}(\dot{y}(t)) d t .
$$

Hence, for $n \leqslant 0 \leqslant m$, we have

$$
\begin{aligned}
u_{m}(x(m))-u_{n}(y(n))= & \int_{n}^{0} L(t, y(t), \dot{y}(t))-c_{y(t)}(\dot{y}(t)) d t \\
& +\int_{0}^{m} L(t, x(t), \dot{x}(t))-c_{x(t)}(\dot{x}(t)) d t .
\end{aligned}
$$

As a consequence, the curve obtained by gluing $y$ on $\mathbb{R}^{-}$and $x$ on $\mathbb{R}^{+}$is the projection of a Hamiltonian trajectory, which, by Cauchy-Lipschitz uniqueness, has to be $P(t)$. In other words, we have proved that $x(t)=y(t)$ on $\mathbb{R}^{-}$. The relation of calibration is now established.

(iii) $\Rightarrow($ ii $)$. Let $P(t)$ be a curve satisfying (iii). Then we have, for $m \geqslant n \geqslant k$,

$$
\begin{aligned}
& \int_{n}^{m} L(t, x(t), \dot{x}(t))-c_{x(t)}(\dot{x}(t)) d t+(m-n) \alpha(c) \\
& \quad=A_{c}(k, x(k) ; m, x(m))+(m-k) \alpha(c)-A_{c}(k, x(k) ; n, x(n))+(n-k) \alpha(c) .
\end{aligned}
$$

Let us denote by $u_{k}^{n}$ the function

$$
u_{k}^{n}(x)=A_{c}(k, x(k) ; n, x)+(n-k) \alpha(c) ;
$$

we obviously have $T_{c}^{m-n} u_{k}^{n}+(m-n) \alpha(c)=u_{k}^{m}$ for $m \geqslant n \geqslant k$. By diagonal extraction, we find an increasing sequence of integers $n_{k}$ such that $u_{-n_{k}}^{n}$ has a limit $u_{n}$ for each $n$ as $k \longrightarrow \infty$. We then have $T_{c}^{m-n} u_{n}+(m-n) \alpha(c)=u_{m}$ for each 
$m \geqslant n$, so that $\mathcal{G}_{c, u_{n}} \in \mathbb{O}$. In addition, we have

$$
\int_{n}^{m} L(t, x(t), \dot{x}(t))-c_{x(t)}(\dot{x}(t)) d t+(m-n) \alpha(c)=u_{m}(x(m))-u_{n}(x(n)) .
$$

$($ ii $) \Rightarrow($ iii $)$ is clear.

(3.14) Lemma. For each $P \in \tilde{\mathcal{E}}(c)$, the orbit $\phi^{n}(P)$ is $\alpha$-asymptotic and $\omega$ asymptotic to the Aubry set $\tilde{\mathcal{A}}(c)$. As a consequence, the Mather set $\tilde{\mathcal{M}}(c)$ is the closure of the union of the supports of the invariant measures of the action of $\phi$ on $\tilde{\mathcal{E}}(c)$

Proof. Let $P(t)=(x(t), p(t))$ be the Hamiltonian orbit of $P$. Let $u_{n}, n \in \mathbb{Z}$, be a sequence of continuous functions such that, for $m \geqslant n$, we have $u_{m}=T_{c}^{m-n} u_{n}+$ $(m-n) \alpha(c)$ and

$$
u_{m}(x(m))-u_{n}(x(n))=\int_{n}^{m} L(t, x(t), \dot{x}(t))-c_{x(t)}(\dot{x}(t)) d t+(m-n) \alpha(c) .
$$

The sequence $u_{m}, m \in \mathbb{Z}$, is equi-semi-concave, hence equi-Lipschitz. Together with (3.1), this implies that this sequence is equi-bounded. If $v$ is a weak KAM solution, that is, a fixed point of $T_{c}-\alpha(c)$, we have, for $m \geqslant n$,

$$
v(x(m))-v(x(n)) \leqslant \int_{n}^{m} L(t, x(t), \dot{x}(t))-c_{x(t)}(\dot{x}(t)) d t+(m-n) \alpha(c) .
$$

It follows that the sequence $n \longmapsto u_{n}(x(n))-v(x(n))$ is non-decreasing and bounded, so that it has a limit $l$ as $n \longrightarrow-\infty$. Let us now consider an increasing sequence $n_{k}$ of integers such that the sequence $P\left(t-n_{k}\right)$ converges, uniformly on compact sets, to a limit $Z(t)=(y(t), z(t))$, which is a Hamiltonian trajectory. Extracting a subsequence in $n_{k}$, we can suppose that there exists a sequence $w_{n}$ of continuous functions on $M$ such that $u_{n-n_{k}} \longrightarrow w_{n}$ uniformly, for each $n$, as $k \longrightarrow \infty$. Then, the sequence $w_{n}$ satisfies $T_{c}^{m-n} w_{n}=w_{m}$ for $m \geqslant n$. In addition, we have $w_{n}(y(n))-v(y(n))=l$ and, for $m \geqslant n$,

$$
w_{m}(y(m))-w_{n}(y(n))=\int_{n}^{m} L(t, y(t), \dot{y}(t))-c_{y(t)}(\dot{y}(t)) d t+(m-n) \alpha(c) .
$$

It follows that, for $m \geqslant n$,

$$
v(x(m))-v(x(n))=\int_{n}^{m} L(t, y(t), \dot{y}(t))-c_{y(t)}(\dot{y}(t)) d t+(m-n) \alpha(c),
$$

which implies that $Z(\mathbb{Z}) \in \tilde{\mathcal{I}}\left(\mathcal{G}_{c, v}\right)$. Since this holds for all weak KAM solutions $v$, we obtain that $Z(\mathbb{Z}) \in \tilde{\mathcal{A}}(c)$. We have proved that the trajectory $P(t)$ is $\alpha$ asymptotic to $\tilde{\mathcal{A}}(c)$. Similarly, one can prove that it is also $\omega$-asymptotic to $\tilde{\mathcal{A}}(c)$. This implies that the invariant measures of the action of $\phi$ on $\tilde{\mathcal{E}}(c)$ are supported on $\tilde{\mathcal{A}}(c)$.

\section{Static classes and heteroclinics}

In this section, we see that there is a natural partition of the Aubry set in compact invariant subsets, which we call static classes, following the terminology of Mañé. This partition was first considered by Mather in 24. We also discuss the existence of heteroclinic orbits between these static classes, extending to the non-autonomous case results of Fathi, Contreras and Paternain; see [18 and [11. 
This survey is also an occasion to introduce several technical lemmas and notation to be used later.

(4.1) Lemma. Let $x$ and $y$ be two points in $M$. The following properties are equivalent:

(i) The points $x$ and $y$ are in $\mathcal{A}(c)$, and the function $z \longmapsto h_{c}(x, z)-h_{c}(y, z)$ is constant on $\mathcal{A}(c)$.

(ii) $h_{c}(x, y)+h_{c}(y, x)=0$.

(iii) The points $x$ and $y$ are in $\mathcal{A}(c)$, and, for each pair $(\mathcal{G}, \breve{\mathcal{G}}) \in \mathbb{V}_{c} \times \breve{V}_{c}$, either the set $\mathcal{G} \wedge \breve{\mathcal{G}}$ contains $x$ and $y$ or it contains neither $x$ nor $y$.

If $x$ and $y$ satisfy these properties, we have, for each $z \in M, h_{c}(x, z)=h_{c}(x, y)+$ $h_{c}(y, z)$.

Proof. $(i) \Rightarrow(i i)$. Assuming $(i)$, we evaluate the constant function at $x$ and $y$ and get $h_{c}(x, x)-h_{c}(y, x)=h_{c}(x, y)-h_{c}(y, y)$; hence $h_{c}(x, y)+h_{c}(y, x)=0$.

(ii) $\Rightarrow($ iii $)$. We have $h_{c}(x, x) \leqslant h_{c}(x, y)+h_{c}(y, x)=0$; hence $x \in \mathcal{A}(c)$. Similarly, $y \in \mathcal{A}(c)$. Let us consider $\mathcal{G}=\mathcal{G}_{c, u} \in \mathbb{V}_{c}$ and $\breve{\mathcal{G}}=\mathcal{G}_{c, \breve{u}} \in \breve{\mathbb{V}}_{c}$ such that $x \in \mathcal{G} \wedge \breve{\mathcal{G}}$ (such a pair exists because $x \in \mathcal{A}(c)$ ). We have to prove that $y \in \mathcal{G} \wedge \breve{\mathcal{G}}$. We have the inequalities $u(y) \leqslant u(x)+h_{c}(x, y)$ and $\breve{u}(y) \geqslant \breve{u}(x)-h_{c}(y, x)$. We obtain the inequality

$$
(u-\breve{u})(y) \leqslant(u-\breve{u})(x)+h_{c}(x, y)+h_{c}(y, x) .
$$

As a consequence, if $h_{c}(x, y)+h_{c}(y, x)=0$, then $y$ is also a point of minimum of $u-\breve{u}$, which is the desired result.

$($ iii $) \Rightarrow($ ii $)$. The point $x$ is a point of minimum of the function $h_{c}(x,)+.h_{c}(., x)$. As a consequence, the point $y$ is also a point of minimum for this function, so that $h_{c}(x, y)+h_{c}(y, x)=h_{c}(x, x)+h_{c}(x, x)=0$.

$($ ii $) \Rightarrow(i)$. We have the inequalities

$$
h_{c}(x, z) \leqslant h_{c}(x, y)+h_{c}(y, z) \text { and } h_{c}(y, z) \leqslant h_{c}(y, x)+h_{c}(x, z) .
$$

If $h_{c}(x, y)+h_{c}(y, x)=0$, then these inequalities sum to an equality, hence they are both equalities.

(4.2) The equivalent properties of the Lemma define an equivalence relation on $\mathcal{A}(c)$. We call static classes the classes of equivalence for this relation. In other words, we say that the points $x$ and $y$ of $\mathcal{A}(c)$ belong to the same static class if they satisfy $(i),(i i)$ or (iii) of the Lemma. We usually denote by $\mathcal{S}$ a static class, and by $\mathcal{S}(x)$ the static class containing $x$. If $\mathcal{S}$ is a static class, we denote by $\tilde{\mathcal{S}}$ the set of points of $\tilde{\mathcal{A}}(c)$ whose projection on $M$ belongs to $\mathcal{S}$. We will also call static classes the sets of the form $\tilde{\mathcal{S}}$. The static classes $\mathcal{S}$ are compact and partition $\mathcal{A}(c)$, and the static classes $\tilde{\mathcal{S}}$ are compact, invariant, and they partition $\tilde{\mathcal{A}}(c)$. The invariance is a direct consequence of the caracterization ( $i i i)$ of the equivalence relation. To each point $x$ of the Aubry set $\mathcal{A}(c)$, we associate the weak KAM solution $h_{c}(x,$.$) ,$ and we denote by $E_{c, x}$ the associated element of $\mathbb{V}_{c}$. The pseudographs of this form are called elementary solutions of $\mathbb{V}_{c}$. Two points of the same static class give rise to the same elementary solution; we will denote by $E_{c, \mathcal{S}}$ the elementary solution induced by points of $\mathcal{S}$. There is a one to one correspondence between the set of static classes and the set of elementary solutions. We will denote this set by $\mathbb{E}_{c} \subset \mathbb{V}_{c}$. We endow it with the induced metric; it is clearly a compact set for this 
metric. We also denote by $\breve{E}_{c, \mathcal{S}}$ the fixed point of $\breve{\Phi}$ associated to the dual weak KAM solution $-h(., x)$ for $x \in \mathcal{S}$.

(4.3) Proposition. Let $\mathcal{G} \in \mathbb{V}_{c}$ be a fixed point and let $P$ be a point of $\overline{\mathcal{G}}$. The $\alpha$-limit of the orbit $\phi^{n}(P)$ is contained in one static class $\tilde{\mathcal{S}} \subset \tilde{\mathcal{A}}(c)$. We also have $P \in \bar{E}_{c, \mathcal{S}}$. In a similar way, if $P \in \breve{\mathcal{G}} \in \breve{\mathbb{V}}_{c}$, then the $\omega$-limit of the orbit of $P$ is contained in one static class of $\tilde{\mathcal{A}}(c)$.

Proof. Let $\alpha \subset M$ be the projection of the $\alpha$-limit of the orbit of $P \in \mathcal{G}_{c, u}$. Note that this $\alpha$-limit is contained in $\tilde{\mathcal{I}}(\mathcal{G})$, so that it is a Lipschitz graph above $\alpha$. We claim that, for each weak KAM solution or backward weak KAM solution $v$, the function $u-v$ is constant on $\alpha$. Clearly, this implies that $\alpha$ is contained in a static class. In order to prove the claim, we consider the projection $x(t)$ on $M$ of the orbit of $P$. The curve $x(t)$ is calibrated by $u$ on $(-\infty, 0]$; hence the equality

$$
u(x(-m))-u(x(-n))=A_{c}(-n, x(-n),-m, x(-m))
$$

holds for all $n, m \in \mathbb{N}$ such that $m \leqslant n$. On the other hand, if $v$ is a weak KAM solution or a backward weak KAM solution, we have the inequality

$$
v(x(-m))-v(x(-n)) \leqslant A_{c}(-n, x(-n),-m, x(-m))
$$

for all $n, m \in \mathbb{N}$ such that $m \leqslant n$. We deduce that the sequence $(u-v)(x(-n))$ : $\mathbb{N} \longrightarrow \mathbb{R}$ is non-increasing. Now let $y=\lim _{k \rightarrow \infty} x\left(-n_{k}\right)$ and $z=\lim _{k \rightarrow \infty} x\left(-m_{k}\right)$ be two points of $\alpha$. We can suppose that $n_{k} \leqslant m_{k} \leqslant n_{k+1}$ by extracting subsequences. We obtain $(u-v)\left(x\left(-n_{k}\right)\right) \geqslant(u-v)\left(x\left(-m_{k}\right)\right) \geqslant(u-v)\left(x\left(-n_{k+1}\right)\right)$, and at the limit $(u-v)(y) \geqslant(u-v)(z) \geqslant(u-v)(y)$. Hence the function $u-v$ is constant on $\alpha$. This proves the first statement of the Proposition.

Taking $v=h_{c}(\alpha,$.$) , we obtain that the sequence u(x(-n))-h_{c}(\alpha, x(-n)): \mathbb{N} \longrightarrow$ $\mathbb{R}$ is non-increasing. On the other hand, we have $u(x(0))-h_{c}(\alpha, x(0)) \leqslant u(\alpha)$. It follows that the sequence is in fact constant, so that the curve $x(t)$ is calibrated by $E_{c, \mathcal{S}(\alpha)}$ on $(-\infty, 0]$, and, by $(2.8),(x(0), p(0)) \in \bar{E}_{c, \mathcal{S}(\alpha)}$.

Corollary. Let $P \in \tilde{\mathcal{N}}(c)$ be a point whose $\alpha$-limit is contained in $\tilde{\mathcal{S}}$ and whose $\omega$-limit is contained in $\tilde{\mathcal{S}}^{\prime}$. We have

$$
P \in E_{c, \mathcal{S}} \tilde{\wedge} \breve{E}_{c, \mathcal{S}^{\prime}} .
$$

Proof. Let $(x(t), p(t))$ be the orbit of $P$. Let $\alpha$ be an $\alpha$-limit point of the curve $x(t)$ and let $\omega$ be an $\omega$-limit point. It follows from the Proposition that $(x(m), p(m)) \in$ $\bar{E}_{c, \mathcal{S}}$ for each $m \in \mathbb{Z}$. Applying the discussions in the proof of the proposition with the point $P=(x(m), p(m))$ and the functions $u=h_{c}(\alpha,$.$) and v=-h_{c}(., \omega)$, we get that the sequence $h_{c}(\alpha, x(n))+h_{c}(x(n), \omega)$ is non-decreasing on $n \leqslant m$. Since we can take any $m \in \mathbb{Z}$, this sequence is non-decreasing on $\mathbb{Z}$. Taking a sequence $m_{k} \longrightarrow \infty$ such that $x\left(m_{k}\right) \longrightarrow \omega$, we obtain the inequality

$h_{c}(\alpha, \omega) \leqslant h_{c}(\alpha, x(n))+h_{c}(x(n), \omega) \leqslant \lim _{k}\left(h_{c}\left(\alpha, x\left(m_{k}\right)\right)+h_{c}\left(x\left(m_{k}\right), \omega\right)\right)=h_{c}(\alpha, \omega)$.

It follows that, for each $n$,

$$
h_{c}(\alpha, x(n))+h_{c}(x(n), \omega)=h_{c}(\alpha, \omega)=\min _{M} h_{c}(\alpha, .)+h_{c}(., \omega) .
$$

This is precisely saying that

$$
x(n) \in E_{c, \mathcal{S}(\alpha)} \wedge \breve{E}_{c, \mathcal{S}(\omega)} .
$$

Recalling that $(x(n), p(n)) \in \bar{E}_{c, \mathcal{S}}$, we obtain $(x(n), p(n)) \in E_{c, \mathcal{S}(\alpha)} \tilde{\wedge} \breve{E}_{c, \mathcal{S}(\omega)}$. 
(4.4) Lemma. If the static class $\mathcal{S}$ is isolated in $\mathcal{A}(c)$, then there exists a neighborhood $V$ of $\mathcal{S}$ in $M$ such that the $\alpha$-limit of every point $P \in E_{c, \mathcal{S}}$ satisfying $\pi(P) \in V$ is contained in $\mathcal{S}$.

Proof. If the result did not hold true, we could find a sequence $\left(x_{n}, p_{n}\right) \in E_{c, \mathcal{S}}$ that has a limit $(x, p) \in \tilde{\mathcal{S}}$ and a sequence $\alpha_{n}$ of $\alpha$-limit points of $\left(x_{n}, p_{n}\right)$ that has a limit $\alpha$ in $\tilde{\mathcal{A}}(c)-\tilde{\mathcal{S}}$. Note that the orbit $\left(x_{n}(t), p_{n}(t)\right):(-\infty, 0] \longrightarrow T^{*} M$ of $\left(x_{n}, p_{n}\right)$ is contained in $E_{c, \mathcal{S}}$. Hence it is calibrated by the function $h_{c}(x,$.$) ; that is,$

$$
h_{c}\left(x, x_{n}(0)\right)=h_{c}\left(x, x_{n}(-k)\right)+A_{c}\left(-k, x_{n}(-k) ; 0, x_{n}(0)\right)+k \alpha(c)
$$

for all $k \in \mathbb{N}$. At the liminf $k \longrightarrow \infty$, for fixed $n$, we obtain the inequality $h_{c}\left(x, x_{n}\right) \geqslant h_{c}\left(x, \alpha_{n}\right)+h_{c}\left(\alpha_{n}, x_{n}\right)$; hence the equality $h_{c}\left(x, x_{n}\right)=h_{c}\left(x, \alpha_{n}\right)+$ $h_{c}\left(\alpha_{n}, x_{n}\right)$. At the limit $n \longrightarrow \infty$ we get $0=h_{c}(x, x)=h_{c}(x, \alpha)+h_{c}(\alpha, x)$. This is in contradiction with the fact that $\alpha$ and $x$ do not belong to the same static class.

(4.5) Let $\tilde{\mathcal{S}}$ and $\tilde{\mathcal{S}}^{\prime}$ be two different static classes in $\tilde{\mathcal{A}}(c)$. The set $E_{c, \mathcal{S}} \tilde{\wedge} \breve{E}_{c, \mathcal{S}^{\prime}}$ contains $\tilde{\mathcal{S}}$ and $\tilde{\mathcal{S}}^{\prime}$ as well as other orbits of $\tilde{\mathcal{N}}(c)$. The following result is similar to Theorem A of [1].

Proposition. The set $E_{c, \mathcal{S}} \wedge \breve{E}_{c, \mathcal{S}^{\prime}}-\left(\mathcal{S} \cup \mathcal{S}^{\prime}\right)$ is not empty and contains points in every neighborhood of $\mathcal{S}$, as well as in every neighborhood of $\mathcal{S}^{\prime}$. More precisely, if $\mathcal{S}$ and $\mathcal{S}^{\prime}$ are two, possibly equal, static classes, and if $\tilde{\mathcal{K}} \subset \tilde{\mathcal{S}}$ and $\tilde{\mathcal{K}}^{\prime} \subset \tilde{\mathcal{S}}^{\prime}$ are two disjoint compact invariant sets, then the set $E_{c, \mathcal{S}} \wedge \breve{E}_{c, \mathcal{S}^{\prime}}-\left(\mathcal{K} \cup \mathcal{K}^{\prime}\right)$ contains points in every neighborhood of $\mathcal{K}$, as well as in every neighborhood of $\mathcal{K}^{\prime}$.

Proof. Let $V$ be an open neighborhood of $\mathcal{K}$ in $M$ which does not intersect $\mathcal{S}^{\prime}$. Let us fix a recurrent orbit $(y(t), z(t)): \mathbb{R} \longrightarrow T^{*} M$ such that $(y(0), z(0))=(y, z) \in \tilde{\mathcal{K}}$ and a recurrent orbit $\left(y^{\prime}(t), z^{\prime}(t)\right): \mathbb{R} \longrightarrow T^{*} M$ such that $\left(y^{\prime}(0), z^{\prime}(0)\right)=\left(y^{\prime}, z^{\prime}\right) \in$ $\tilde{\mathcal{K}}^{\prime}$. Consider a sequence $n_{k} \in \mathbb{N}$ of integers and a sequence $\left(x_{k}(t), p_{k}(t)\right):\left[0, n_{k}\right] \longrightarrow$ $T^{*} M$ of Hamiltonian trajectories such that $x_{k}(0)=y$ and $x_{k}\left(n_{k}\right)=y^{\prime}$ and

$$
\int_{0}^{n_{k}} L\left(t, x_{k}(t), \dot{x}_{k}(t)\right)-c_{x_{k}(t)}\left(\dot{x}_{k}(t)\right)+\alpha(c) d t=A_{c}\left(0, y ; n_{k}, y^{\prime}\right)+n_{k} \alpha(c) \longrightarrow h_{c}\left(y, y^{\prime}\right) .
$$

We extend the curve $x_{k}:\left[0, n_{k}\right] \longrightarrow M$ to a curve $x_{k}: \mathbb{R} \longrightarrow \mathbb{R}$ by setting $x_{k}(t)=y(t)$ for $t \leqslant 0$ and $x_{k}(t)=y^{\prime}\left(t-n_{k}\right)$ for $t \geqslant n_{k}$. Let $a_{k}$ and $b_{k}$ be two increasing sequences of integers such that $y\left(-a_{k}\right) \longrightarrow y$ and $y^{\prime}\left(b_{k}\right) \longrightarrow y^{\prime}$. The existence of such sequences follows from the fact that the curves $y(t)$ and $y^{\prime}(t)$ are recurrent. Since the curve $y(t)$ is calibrated by $h_{c}(y,$.$) , we have, as k \longrightarrow \infty$,

$$
A_{c}\left(-a_{k}, y\left(-a_{k}\right) ; 0, y\right)+a_{k} \alpha(c)=-h_{c}\left(y, y\left(-a_{k}\right)\right) \longrightarrow 0,
$$

and similarly

$$
A_{c}\left(0, y^{\prime} ; b_{k}, y_{k}\left(b_{k}\right)\right)+b_{k} \alpha(c)=h_{c}\left(y^{\prime}, y\left(b_{k}\right)\right) \longrightarrow 0 .
$$

As a consequence, we have, as $k \longrightarrow \infty$,

$$
A_{c}\left(-a_{k}, x_{k}\left(-a_{k}\right) ; b_{k}+n_{k}, x_{k}\left(b_{k}+n_{k}\right)\right)+\left(b_{k}+a_{k}+n_{k}\right) \alpha(c) \longrightarrow h_{c}\left(y, y^{\prime}\right) .
$$

For each $k$, let $T_{k}$ be the maximum of all times $i \in \mathbb{N}$ such that $x_{k}(i) \in V$. Note that $x_{k}\left(T_{k}+1\right)$ does not belong to $V$. We can assume, taking a subsequence, that the 
curve $x_{k}\left(t+T_{k}\right)$ is converging uniformly on compact sets to a limit $x(t): \mathbb{R} \longrightarrow M$. Let us now fix $m \leqslant n$ in $\mathbb{Z}$. Summing the inequalities

$$
\begin{gathered}
\liminf _{k \longrightarrow \infty}\left(A_{c}\left(-a_{k}, x_{k}\left(-a_{k}\right) ; T_{k}+m, x_{k}\left(T_{k}+m\right)\right)+\left(T_{k}+m+a_{k}\right) \alpha(c)\right) \geqslant h_{c}(y, x(m)), \\
\liminf _{k \longrightarrow \infty}\left(A_{c}\left(T_{k}+m, x_{k}\left(T_{k}+m\right) ; T_{k}+n, x_{k}\left(T_{k}+n\right)\right)\right)=A_{c}(m, x(m) ; n, x(n))
\end{gathered}
$$

and

$$
\begin{aligned}
\liminf _{k \longrightarrow \infty} & \left(A_{c}\left(T_{k}+n, x_{k}\left(T_{k}+n\right) ; b_{k}+n_{k}, x_{k}\left(b_{k}+n_{k}\right)\right)+\left(b_{k}+n_{k}-T_{k}-n\right) \alpha(c)\right) \\
& \geqslant h_{c}\left(x(n), y^{\prime}\right)
\end{aligned}
$$

we get

$$
\begin{aligned}
h\left(y, y^{\prime}\right) & =\liminf _{k \longrightarrow \infty} A_{c}\left(-a_{k}, x_{k}\left(-a_{k}\right) ; b_{k}+n_{k}, x_{k}\left(b_{k}+n_{k}\right)\right)+\left(b_{k}+a_{k}+n_{k}\right) \alpha(c) \\
& \geqslant h_{c}(y, x(m))+A_{c}(m, x(m) ; n, x(n))+(n-m) \alpha(c)+h_{c}\left(x(n), y^{\prime}\right) .
\end{aligned}
$$

Since the converse inequality obviously holds, we obtain the equality

$$
h_{c}\left(y, y^{\prime}\right)=h_{c}(y, x(m))+A_{c}(m, x(m) ; n, x(n))+(m-n) \alpha(c)+h_{c}\left(x(n), y^{\prime}\right)
$$

for all $m \leqslant n$. It follows that all the inequalities above are in fact equalities, so that we also have

$$
h_{c}(y, x(m))+A_{c}(m, x(m) ; n, x(n))+(m-n) \alpha(c)=h_{c}(y, x(n))
$$

so that the orbit $x(t)$ is calibrated by the weak $\mathrm{KAM}$ solution $h_{c}(y,$.$) on \mathbb{R}$. Hence it is the projection of a Hamiltonian trajectory $(x(t), p(t))$. Moreover, we have the equality

$$
h_{c}\left(y, y^{\prime}\right)=h_{c}(y, x(n))+h_{c}\left(x(n), y^{\prime}\right),
$$

so that the point $x(n)$ is a point of minimum of the function $h_{c}(y,)+.h_{c}\left(., y^{\prime}\right)$. Hence it belongs to $E_{c, \mathcal{S}} \wedge \breve{E}_{c, \mathcal{S}^{\prime}}$. We have proved that the sequence $(x(n), p(n)), n \in \mathbb{Z}$, is an orbit of $\phi$ which is contained in the invariant graph $E_{c, \mathcal{S}} \tilde{\wedge} \breve{E}_{c, \mathcal{S}}$. Since the point $x(1)$ is not a point of $\mathcal{K}$, this orbit does not intersect the invariant set $\tilde{\mathcal{K}}$. As a consequence, the point $x(0)$ belongs to $\bar{V}-\mathcal{K}$. We have proved that the set $E_{c, \mathcal{S}} \wedge \breve{E}_{c, \mathcal{S}^{\prime}}-\left(\mathcal{K} \cup \mathcal{K}^{\prime}\right)$ contains points in each neighborhood of $\mathcal{K}$. One can prove in a similar way that this set contains points in every neighborhood of $\mathcal{K}^{\prime}$.

(4.6) Corollary. A static class $\tilde{\mathcal{S}}$ cannot be decomposed as the union of two disjoint invariant compact subsets.

Proof. Assume, by contradiction, that there exists a static class $\tilde{\mathcal{S}}=\tilde{\mathcal{K}}_{1} \cup \tilde{\mathcal{K}}_{2}$, with $\tilde{\mathcal{K}}_{i}$ invariant, compact and disjoint. In view of (4.5), the set

$$
E_{c, \mathcal{S}} \wedge \breve{E}_{c, \mathcal{S}}-\left(\mathcal{K}_{1} \cup \mathcal{K}_{2}\right)
$$

is not empty. This is a contradiction since $E_{c, \mathcal{S}} \wedge \breve{E}_{c, \mathcal{S}}=\mathcal{S}$ and $\mathcal{K}_{1} \cup \mathcal{K}_{2}=\mathcal{S}$.

(4.7) Let $(x(t), p(t)): \mathbb{R} \longrightarrow T^{*} M$ be an orbit of the Mañé set, that is, an orbit satisfying $(x(0), p(0)) \in \tilde{\mathcal{N}}(c)$. This orbit is $\alpha$-asymptotic to a static class $\tilde{\mathcal{S}}$, and $\omega$-asymptotic to a static class $\tilde{\mathcal{S}}^{\prime}$.

Lemma. The inclusion $(x(0), p(0)) \in \tilde{\mathcal{A}}(c)$ holds if and only if $\mathcal{S}=\mathcal{S}^{\prime}$. In this case, we have $(x(0), p(0)) \in \tilde{\mathcal{S}}$. 
Proof. Let us first assume that $\mathcal{S}=\mathcal{S}^{\prime}$. In this case, we see from Corollary (4.3) that $(x(0), p(0)) \in E_{c, \mathcal{S}} \tilde{\wedge} \breve{E}_{c, \mathcal{S}}$. But is is clear from the definition of static classes that $E_{c, \mathcal{S}} \tilde{\wedge} \breve{E}_{c, \mathcal{S}}=\tilde{\mathcal{S}}$. Consequently, we have $(x(0), p(0)) \in \tilde{\mathcal{S}} \subset \tilde{\mathcal{A}}(c)$. Conversely, assume that $(x(0), p(0)) \in \tilde{\mathcal{A}}(c)$. Then this point is contained in one static class $\tilde{\mathcal{S}}_{0}$. Since this static class is compact and invariant, it contains the $\alpha$ - and the $\omega$-limits of the orbit $(x(t), p(t))$, so that we have $\tilde{\mathcal{S}}=\tilde{\mathcal{S}}_{0}=\tilde{\mathcal{S}}^{\prime}$.

Corollary. We have the equality $\tilde{\mathcal{A}}(c)=\tilde{\mathcal{N}}(c)$ if and only if there is exactly one static class in $\tilde{\mathcal{A}}(c)$.

(4.8) Let $\tilde{\mathcal{H}}_{c}\left(\tilde{\mathcal{S}}, \tilde{\mathcal{S}}^{\prime}\right)$ be the set of orbits of $\tilde{\mathcal{N}}(c)$ which are heteroclinic orbits between the static classes $\tilde{\mathcal{S}}$ and $\tilde{\mathcal{S}}^{\prime}$; we denote by $\mathcal{H}_{c}\left(\mathcal{S}, \mathcal{S}^{\prime}\right)$ its projection on $M$. We have

$$
\tilde{\mathcal{N}}(c)=\tilde{\mathcal{A}}(c) \cup \bigcup_{\mathcal{S}, \mathcal{S}^{\prime}} \tilde{\mathcal{H}}_{c}\left(\mathcal{S}, \mathcal{S}^{\prime}\right),
$$

where the union is taken on all pairs $\left(\mathcal{S}, \mathcal{S}^{\prime}\right)$ of different static classes. Recall from Corollary (4.3), that

$$
\tilde{\mathcal{H}}_{c}\left(\tilde{\mathcal{S}}, \tilde{\mathcal{S}}^{\prime}\right) \subset E_{c, s} \tilde{\wedge} \breve{E}_{c, \mathcal{S}^{\prime}}
$$

The following result is from [18] and [1].

(4.9) Proposition. If the static class $\tilde{\mathcal{S}}$ is properly contained and isolated in $\tilde{\mathcal{A}}(c)$, then there exists an orbit of $\phi$ in $\tilde{\mathcal{N}}(c)-\tilde{\mathcal{A}}(c)$ which is $\alpha$-asymptotic to $\tilde{\mathcal{S}}$. This orbit is then $\omega$-asymptotic to another static class $\tilde{\mathcal{S}}^{\prime}$.

Proof. Let us choose, according to (4.4), a neighborhood $V$ of $\mathcal{S}$ such that every orbit of $E_{c, \mathcal{S}}$ starting above $V$ has its $\alpha$-limit contained in $\mathcal{S}$. Now let us choose any static class $\mathcal{S}^{\prime \prime}$ different from $\mathcal{S}$. In view of (4.5) the set $E_{c, \mathcal{S}} \wedge \breve{E}_{c, \mathcal{S}^{\prime \prime}}$ intersects $V-\mathcal{S}$. Let $P(t)=(x(t), p(t)): \mathbb{R} \longrightarrow T^{*} M$ be an orbit such that $P(0) \in E_{c, \mathcal{S}} \tilde{\wedge} \breve{E}_{c, \mathcal{S}^{\prime \prime}}$ and $x(0) \in V-\mathcal{S}$. The $\alpha$-limit of the orbit $P(t)$ is contained in $\tilde{\mathcal{S}}$. On the other hand, this orbits belongs to $\tilde{\mathcal{N}}(c)$; hence its $\omega$-limit is contained in some static class $\tilde{\mathcal{S}}^{\prime}$.

(4.10) So far we have treated the case where there exist several static classes. We recall, however, that the existence of a single static class in $\mathcal{A}(c)$, is, for $c$ fixed, a generic property of the Lagrangian; see 11. We will explain in Section 7 a device due to Fathi, as well as Contreras and Paternain (see [18 and [11]) which allows us to treat this case.

\section{ABStract MECHANISMS}

\section{The relation and its dynamical consequences}

We define the forcing relation $\dashv$ and describe its dynamical consequences. We prove Proposition (0.10).

(5.1) Let us introduce some useful notation. Given two subsets $\mathcal{G}$ and $\mathcal{G}^{\prime}$ of $T^{*} M$, we define the relation $\mathcal{G} \vdash_{N} \mathcal{G}^{\prime}$ as follows:

$$
\mathcal{G} \vdash_{N} \mathcal{G}^{\prime} \Longleftrightarrow \overline{\mathcal{G}}^{\prime} \subset \bigcup_{n=1}^{N} \phi^{n}(\mathcal{G}),
$$


where as usual $\overline{\mathcal{G}}$ is the closure of $\mathcal{G}$. We say that $\mathcal{G}$ forces $\mathcal{G}^{\prime}$, and write $\mathcal{G} \vdash \mathcal{G}^{\prime}$ if there exists an integer $N$ such that $\mathcal{G} \vdash_{N} \mathcal{G}^{\prime}$. If $\mathcal{G}$ is a subset of $T^{*} M$ and if $c \in H^{1}(M, \mathbb{R})$, the relations

$$
\mathcal{G} \vdash c \text { and } \mathcal{G} \vdash{ }_{N} c
$$

mean that there exists an overlapping pseudograph $\mathcal{G}^{\prime}$ of cohomology $c$ such that $\mathcal{G} \vdash \mathcal{G}^{\prime}$ (resp. $\mathcal{G} \vdash{ }_{N} \mathcal{G}^{\prime}$ ). To finish, for $c$ and $c^{\prime}$ two cohomology classes, the relation

$$
c \vdash_{N} c^{\prime}
$$

means that, for each pseudograph $\mathcal{G} \in \mathbb{P}_{c}$, we have $\mathcal{G} \vdash_{N} c^{\prime}$. As the reader may have guessed, we will then say that $c$ forces $c^{\prime}\left(c \vdash c^{\prime}\right)$ if there exists an integer $N$ such that $c \vdash_{N} c^{\prime}$. The relation $\vdash$ (between subsets as well as between cohomology classes) is obviously transitive. In this paper we will be concerned with understanding the relation $\vdash$ between cohomology classes. For this purpose, it is useful to introduce the symmetric relation

$$
c \dashv c^{\prime} \Longleftrightarrow c \vdash c^{\prime} \text { and } c^{\prime} \vdash c .
$$

We say that $c$ and $c^{\prime}$ force each other if $c-1 c^{\prime}$.

Proposition. The forcing relation $\dashv$ is an equivalence relation on $H^{1}(M, \mathbb{R})$.

Note that we have $c \vdash_{1} c$ for each $c$ since $\overline{\Phi(\mathcal{G})} \subset \phi(\mathcal{G})$ for each $\mathcal{G} \in \mathbb{P}_{c}$, which can be written $\mathcal{G} \vdash_{1} \Phi(\mathcal{G})$.

(5.2) Let us present a simple (negative) result about this relation. If $\mathcal{G}$ is a graph of a continuous section of $T^{*} M$, and is invariant under $\phi$, then $\mathcal{G} \in \mathbb{V} \cap \breve{\mathbb{V}}$ is in fact a Lipschitz graph, and the relation $c(\mathcal{G}) \vdash c$ holds if and only if $c=c(\mathcal{G})$. Note that, if $C \subset H^{1}(M, \mathbb{R})$ is bounded, it is possible to choose a uniform constant $K$ such that all the invariant Lipschitz Graphs $\mathcal{G}$ whose cohomology satisfies $c \in C$ are $K$-Lipschitz. In other words, the elements of $\mathbb{V}_{C} \cap \breve{V}$ are equi-Lipschitz graphs. Of course, we would like to be able to prove that the forcing relation $\dashv \Vdash$ has non-trivial classes. We first restate and prove Proposition (0.10)

\section{(5.3) Proposition.}

(i) If the cohomology class $c$ forces the cohomology class $c^{\prime}$, there exists a heteroclinic trajectory of the Hamiltonian flow between $\tilde{\mathcal{A}}(c)$ and $\tilde{\mathcal{A}}\left(c^{\prime}\right)$. For any closed forms $\eta$ of cohomology $c$ and $\eta^{\prime}$ of cohomology $c^{\prime}$, there exists a positive integer $N$ and a trajectory $(q(t), p(t)):[0, N] \longrightarrow T^{*} M$ of the Hamiltonian flow such that $p(0)=\eta_{q(0)}$ and $p(N)=\eta_{q(N)}^{\prime}$. There exists a trajectory $(q(t), p(t)):[0, \infty) \longrightarrow T^{*} M$ which satisfies $p(0)=\eta_{q(0)}$ and is $\omega$ asymptotic to $\tilde{\mathcal{A}}\left(c^{\prime}\right)$. There exists a trajectory $(q(t), p(t)):(-\infty, 0] \longrightarrow T^{*} M$ which satisfies $p(0)=\eta_{q(0)}^{\prime}$ and is $\alpha$-asymptotic to $\tilde{\mathcal{A}}(c)$.

(ii) Let $c_{i}, i \in \mathbb{Z}$, be a sequence of cohomology classes such that $c_{i}$ forces $c_{i+1}$ for each $i \in \mathbb{Z}$. Fix for each $i$ a neighborhood $U_{i}$ of $\tilde{\mathcal{M}}\left(c_{i}\right)$ in $T^{*} M$. There exists a trajectory of the Hamiltonian flow which in turn visits all the sets $U_{i}$. In addition, if the sequence stabilizes to $c-$ on the left, or to $c+$ on the right, the trajectory can be assumed negatively asymptotic to $\mathcal{A}(c-)$ or positively asymptotic to $\mathcal{A}(c+)$.

Proof. Let us first assume that $c \vdash c^{\prime}$. Take a fixed point $\mathcal{G}_{c} \in \mathbb{V}_{c}$. There exists a graph $\mathcal{G} \in \mathbb{P}_{c^{\prime}}$ such that $\mathcal{G}_{c} \vdash \mathcal{G}$. Now, consider a pseudograph $\breve{\mathcal{G}}_{c^{\prime}} \in \breve{\mathbb{V}}_{c^{\prime}}$. It follows from Lemma (2.3) that $\mathcal{G}$ intersects $\breve{\mathcal{G}}_{c^{\prime}}$. In view of (4.3) the points of intersection 
are $\alpha$-asymptotic to $\tilde{\mathcal{A}}(c)$ and $\omega$-asymptotic to $\tilde{\mathcal{A}}\left(c^{\prime}\right)$. In the same way, we can take for $\mathcal{G}_{c}$ the graph of the closed form $\eta$, choose $\mathcal{G} \in \mathbb{P}_{c^{\prime}}$ such that $\mathcal{G}_{c} \vdash \mathcal{G}$, and take for $\breve{\mathcal{G}}_{c^{\prime}}$ the graph of $\eta^{\prime}$. The points of the intersection $\mathcal{G} \cap \breve{\mathcal{G}}_{c^{\prime}}$ have trajectories from $\mathcal{G}_{c}$ to $\breve{\mathcal{G}}_{c^{\prime}}$. The other statements of $(i)$ are proved similarly.

(5.4) Lemma. Let us fix a cohomology c.

(i) For each neighborhood $U$ of $\tilde{\mathcal{E}}(c)$, there exists $N \in \mathbb{N}$ such that, for all $l \geqslant N$ and all $\mathcal{G} \in \mathbb{P}_{c}$, we have

$$
\phi^{-l}\left(\overline{\Phi^{2 l}(\mathcal{G})}\right) \subset U
$$

(ii) If $V$ is an open neighborhood of $\tilde{\mathcal{M}}(c)$ in $T^{*} M$, there exists $N \in \mathbb{N}$ such that, for each $\mathcal{G} \in \mathbb{P}_{c}$ and each $P \in \overline{\Phi^{N}(\mathcal{G})}$, one of the points $\phi^{-i}(P), 1 \leqslant$ $i \leqslant N-1$, belongs to $V$.

Proof. In order to prove $(i)$, it is sufficient to prove that if $\mathcal{G}_{n} \in \mathbb{P}_{c}$ is a sequence of pseudographs, if $m_{n}$ is an increasing sequence of integers, and if $\left(x_{n}(t), p_{n}(t)\right)$ : $\left[0, m_{n}\right] \longrightarrow T^{*} M$ is a Hamiltonian trajectory which satisfies

$$
\left(x_{n}\left(m_{n}\right), p_{n}\left(m_{n}\right)\right) \in \overline{\Phi^{2 m_{n}}\left(\mathcal{G}_{n}\right)}
$$

and which converges uniformly on compact sets to a limit $(x(t), p(t)): \mathbb{R}^{+} \longrightarrow$ $T^{*} M$, then $(x(0), p(0)) \in \tilde{\mathcal{E}}(c)$.

Let us write the pseudographs $\Phi^{m_{n}}\left(\mathcal{G}_{n}\right)$ on the form $\mathcal{G}_{c, u_{n}}$. For each $k, n \in \mathbb{N}$, we have

$$
T_{c}^{k} u_{n}\left(x_{n}(k)\right)=u_{n}\left(x_{n}(0)\right)+\int_{0}^{k} L\left(t, x_{n}(t), \dot{x}_{n}(t)\right)+c_{x_{n}(t)}\left(\dot{x}_{n}(t)\right) d t .
$$

Since the functions $u_{n}$ lie in the image of the operator $T_{c}^{m_{n}}$, they are equi-Lipschitz, and there exists a real sequence $\lambda_{n}$ such that the sequence of functions $\lambda_{n}+u_{n}$ has accumulation points in $C(M, \mathbb{R})$. As a consequence, we can assume, taking a subsequence if necessary, that the functions $\lambda_{n}+u_{n}$ converge uniformly to a limit $u$. We have $\mathcal{G}_{c, u}=\lim \Phi^{m_{n}}\left(\mathcal{G}_{n}\right) \in \mathbb{O}_{c}$. For each fixed $k \in \mathbb{Z}$, taking the limit as $n \longrightarrow \infty$, we get

$$
T_{c}^{k} u(x(k))=u(x(0))+\int_{0}^{k} L(t, x(t), \dot{x}(t))+c_{x(t)}(\dot{x}(t)) d t .
$$

Hence we have $P(k-1) \in \Phi^{k-1}\left(\mathcal{G}_{c, u}\right)$, and therefore $P(0) \in \phi^{1-k}\left(\Phi^{k-1}\left(\mathcal{G}_{c, u}\right)\right)$. Since this holds for all $k \in \mathbb{N}$, we conclude that

$$
P(0) \in \tilde{\mathcal{I}}\left(\mathcal{G}_{c, u}\right) \subset \tilde{\mathcal{E}}(c) .
$$

In order to prove $(i i)$, it is useful to recall that $\tilde{\mathcal{E}}(c)$ is a compact set, invariant under the time-one flow $\phi$, and that the Mather set $\tilde{\mathcal{M}}(c)$ is the union of the supports of the invariant measures of the action of $\phi$ on $\tilde{\mathcal{E}}(c)$. The claim below follows from general facts about topological dynamics on compact spaces: For each neighborhood $W$ of $\tilde{\mathcal{M}}(c)$ in $\tilde{\mathcal{E}}(c)$, there exists an integer $k$ such that, for each point $P$ of $\tilde{\mathcal{E}}(c)$, one of the points $\phi^{i}(P), 1 \leqslant i \leqslant k$, belongs to $W$. As a consequence, if $V$ is a neighborhood of $\tilde{\mathcal{M}}(c)$ in $T^{*} M$, there exists a neighborhood $U$ of $\tilde{\mathcal{E}}(c)$ in $T^{*} M$ such that, for each $P \in U$, one of the points $\phi^{i}(P), 1 \leqslant i \leqslant k$, belongs to $V$. Now let us take $l \geqslant k$ such that $(i)$ holds for this neighborhood $U$, and set $N=2 l$. For 
each $\mathcal{G} \in \mathbb{P}_{c}$ and each $P \in \overline{\Phi^{N}(\mathcal{G})}$, we have $\phi^{-l}(P) \in U$. Hence one of the points $\phi^{i-l}(P), 1 \leqslant i \leqslant k$, is in $V$, which proves $(i i)$.

(5.5) Let us now prove $(i i)$ of the Proposition. Let $M_{i} \in \mathbb{N}, i \in \mathbb{Z}$, be a sequence of integers such that $c_{i} \vdash_{M_{i}} c_{i+1}$, and let $W_{i} \subset V_{i}$ be compact neighborhoods of $\tilde{\mathcal{M}}\left(c_{i}\right)$. In view of Lemma $(5.4)$, there exists a sequence $N_{i}$ of integers such that, for each $\mathcal{G} \in \mathbb{P}_{c_{i}}$ and each

$$
P \in \phi^{-N_{i}}\left(\overline{\Phi^{N_{i}}(\mathcal{G})}\right)
$$

one of the points $\phi^{l}(P), 0 \leqslant l \leqslant N_{i}$, belongs to $W_{i}$.

Let us first fix an integer $k \in \mathbb{N}$ and choose a pseudograph $\mathcal{G}_{-k}^{k} \in \mathbb{P}_{c_{-k}}$. Since $c_{-k} \vdash_{M_{-k}} c_{1-k}$, there exists a pseudograph $\mathcal{G}_{1-k}^{k} \in \mathbb{P}_{c_{1-k}}$ such that $\Phi^{N_{-k}}\left(\mathcal{G}_{-k}^{k}\right) \vdash_{M_{-k}}$ $\mathcal{G}_{1-k}^{k}$. We build, by induction, a sequence $\mathcal{G}_{i}^{k} \in \mathbb{P}_{c_{i}}, i \geqslant-k$, of pseudographs such that

$$
\Phi^{N_{i}}\left(\mathcal{G}_{i}^{k}\right) \vdash_{M_{i}} \mathcal{G}_{i+1}^{k}
$$

for each $i \geqslant-k$.

Let us now take a point $P_{k}^{k} \in \mathcal{G}_{k}^{k}$. There exists a positive integer $l_{k-1}^{k} \leqslant M_{k-1}$ such that $\phi^{-l_{k-1}^{k}}\left(P_{k}^{k}\right) \in \Phi^{N_{k-1}}\left(\mathcal{G}_{k-1}^{k}\right)$. We then set $P_{k-1}^{k}=\phi^{-\left(l_{k-1}^{k}+N_{k-1}\right)}\left(P_{k}^{k}\right)$; this point belongs to $\mathcal{G}_{k-1}^{k}$. We can build a sequence $P_{i}^{k},-k \leqslant i \leqslant k$, of points of $\mathcal{G}_{i}^{k}$ and a sequence $l_{i}^{k},-k \leqslant i \leqslant k-1$, of integers satisfying $0 \leqslant l_{i}^{k} \leqslant M_{i}$ such that

$$
\phi^{N_{i}+l_{i}^{k}}\left(P_{i}^{k}\right)=P_{i+1}^{k}
$$

for each $i$. In addition, one of the points $\phi^{j}\left(P_{i}^{k}\right), 0 \leqslant j \leqslant N_{i}$, belongs to $W_{i}$.

There exists an increasing sequence $k_{n}$ of integers such that each of the sequences $n \longmapsto l_{i}^{k_{n}}$, for fixed $i$, is the constant $l_{i}$ after a certain rank, and each of the sequences $n \longmapsto P_{i}^{k_{n}}$, for fixed $i$, is converging to $P_{i}$. Clearly, we have $\phi^{l_{i}+N_{i}}\left(P_{i}\right)=P_{i+1}$ for each $i \in \mathbb{Z}$, and one of the points $\phi^{j}\left(P_{i}\right), 0 \leqslant j \leqslant N_{i}$, belongs to $W_{i}$. This proves the main part of the statement.

If the sequence $c_{i}$ stabilizes to $c-$ on the right, then it is possible to build a sequence $\mathcal{G}_{i} \in \mathbb{P}_{c_{i}}$ as above which stabilizes to $\mathcal{G}-\in \mathbb{V}_{c-}$ on the right, and we obtain by the above method an orbit which is $\alpha$-asymptotic to $\tilde{\mathcal{A}}(c-)$ and then visits in turn all the sets $W_{i}$. If the sequence $c_{i}$ stabilizes to $c+$ on the right, say for $i \geqslant I$, then it is possible to impose that $P_{I} \in \breve{\mathcal{G}}+\in \breve{\mathbb{V}}_{c+}$ in the construction above, and we then obtain an orbit which is $\omega$-asymptotic to $\tilde{\mathcal{A}}(c+)$.

\section{Evolution operators}

We define operators on $\mathbb{P}$ that generalize the Lax-Oleinik operator $\Phi$. These operators will play a central role in the proof of our main results.

(6.1) Given two integers $N^{\prime} \geqslant N \geqslant 1$, and a cohomology $c$, we define the function $A_{c}^{N, N^{\prime}}: M \times M \longrightarrow \mathbb{R}$ by the expression

$$
A_{c}^{N, N^{\prime}}(x, y)=\min _{k \in \mathbb{N}, N \leqslant k \leqslant N^{\prime}} A_{c}(0, x ; k, y)+k \alpha(c) .
$$

Since each of the mappings

$$
\begin{aligned}
H^{1}(M, \mathbb{R}) & \longrightarrow C(M \times M, \mathbb{R}), \\
c & \longmapsto A_{c}(0, . ; k, .)
\end{aligned}
$$


is continuous (see Appendix $\mathrm{B}$ ), it is easy to see that, for fixed $N^{\prime} \geqslant N$, the mapping

$$
\begin{aligned}
H^{1}(M, \mathbb{R}) & \longrightarrow C(M \times M, \mathbb{R}), \\
c & \longmapsto A_{c}^{N, N^{\prime}}
\end{aligned}
$$

is continuous.

Proposition. Let c be a fixed cohomology class. For each $\epsilon>0$, there exist integers $N^{\prime} \geqslant N \geqslant 1$ such that

$$
\left\|A_{c}^{N, N^{\prime}}-h_{c}\right\|_{\infty} \leqslant \epsilon .
$$

More precisely, there exists an integer $N_{0}$ and a function $\Lambda: \mathbb{N} \longrightarrow \mathbb{N}$ such that the conclusion holds if $N \geqslant N_{0}$ and $N^{\prime} \geqslant \Lambda(N)$.

Proof. The functions $A_{c}^{N, N^{\prime}}, N \leqslant N^{\prime} \in \mathbb{N}$, and the function $h_{c}$ have a common modulus of continuity and a common uniform bound. As a consequence, the pointwise limit

is uniform.

$$
h_{c}(x, y)=\lim _{N \longrightarrow \infty} \lim _{N^{\prime} \longrightarrow \infty} A_{c}^{N, N^{\prime}}(x, y)
$$

(6.2) It is useful to generalize the operators $\Phi^{N}: \mathbb{P} \longrightarrow \mathbb{P}$. Given two integers $N^{\prime} \geqslant N \geqslant 1$ and an open set $U \subset M$, we define the operator

$$
\Phi_{U}^{N, N^{\prime}}: \mathbb{P} \longrightarrow \mathbb{P}
$$

by the relation $\Phi_{U}^{N, N^{\prime}}\left(\mathcal{G}_{c, u}\right)=\mathcal{G}_{c, T_{c, U}^{N, N^{\prime}} u}$ where

$$
T_{c, U}^{N, N^{\prime}} u(x)=\min _{y \in \bar{U}, N \leqslant k \leqslant N^{\prime}} T_{c}^{k} u(y)+k \alpha(c)=\min _{y \in \bar{U}} u(y)+A_{c}^{N, N^{\prime}}(y, x) .
$$

For simplicity we will denote by $\Phi^{N, N^{\prime}}$ the operator $\Phi_{M}^{N, N^{\prime}}$. For each $\mathcal{G} \in \mathbb{P}$, we have

$$
\mathcal{G} \vdash_{N^{\prime}} \Phi^{N, N^{\prime}}(\mathcal{G}) .
$$

Lemma. For each integer $1 \leqslant N \leqslant N^{\prime}$ and each open set $U \subset M$, the operator $\Phi_{U}^{N, N^{\prime}}: \mathbb{P} \longrightarrow \mathbb{P}$ is continuous when the source is endowed with the seminorm $\|.\|_{U}$ and the image with the norm $\|$.$\| ; see (2.2).$

Proof. Let $\mathcal{G}=\mathcal{G}_{c, u}$ and $\mathcal{G}_{1}=\mathcal{G}_{c_{1}, u_{1}}$ be two pseudographs. We have

$$
\left\|\Phi_{U}^{N, N^{\prime}}\left(\mathcal{G}_{1}\right)-\Phi_{U}^{N, N^{\prime}}(\mathcal{G})\right\| \leqslant\left|c_{1}-c\right|+\left\|T_{c_{1}, U}^{N, N^{\prime}} u_{1}-T_{c, U}^{N, N^{\prime}} u\right\| .
$$

In order to estimate the term $\left\|T_{c_{1}, U}^{N, N^{\prime}} u_{1}-T_{c, U}^{N, N^{\prime}} u\right\|$, let us write

$$
T_{c, U}^{N, N^{\prime}} u=u(y)+A_{c}^{N, N^{\prime}}(y, x)
$$

with $y \in \bar{U}$. Then, we have

$$
T_{c_{1}, U}^{N, N^{\prime}} u_{1}-T_{c, U}^{N, N^{\prime}} u \leqslant u_{1}(y)+A_{c_{1}}^{N, N^{\prime}}(y, x)-u(y)-A_{c}^{N, N^{\prime}}(y, x),
$$

and by symmetry

$$
\left\|T_{c_{1}, U}^{N, N^{\prime}} u_{1}-T_{c, U}^{N, N^{\prime}} u\right\| \leqslant \sup _{y \in U}\left|u_{1}(y)-u(y)\right|+\left\|A_{c_{1}}^{N, N^{\prime}}-A_{c}^{N, N^{\prime}}\right\| .
$$

The conclusion follows from the continuity of the mapping $c \longmapsto A_{c}^{N, N^{\prime}}$; see (6.1). 
(6.3) Similarly, we define the operator

$$
\Phi_{U}^{\infty}: \mathbb{P} \longrightarrow \mathbb{V}
$$

by the relation $\Phi_{U}^{\infty}\left(\mathcal{G}_{c, u}\right)=\mathcal{G}_{c, T_{c, U}^{\infty} u}$ where

$$
T_{c, U}^{\infty} u(x)=\min _{y \in \bar{U}} u(y)+h_{c}(y, x) .
$$

In the autonomous case Fathi proved that the sequence $\Phi^{n}(\mathcal{G})$ is converging to a fixed point of $\Phi$ for each $\mathcal{G} \in \mathbb{P}$. Such a result would be very useful to us, but does not hold in our non-autonomous setting. It is replaced by the following one.

Proposition. Let $c$ be a fixed cohomology class. For each $\epsilon>0$ there exist integers $N \leqslant N^{\prime}$ such that, for each pseudograph $\mathcal{G}=\mathcal{G}_{c, u} \in \mathbb{P}_{c}$ and each open set $U \subset M$, we have

$$
\left\|\Phi_{U}^{N, N^{\prime}}(\mathcal{G})-\Phi_{U}^{\infty}(\mathcal{G})\right\| \leqslant \epsilon .
$$

More precisely, there exists an integer $N_{0}$ and a function $\Lambda: \mathbb{N} \longrightarrow \mathbb{N}$ such that the conclusion holds if $N \geqslant N_{0}$ and $N^{\prime} \geqslant \Lambda(N)$.

Proof. It is not hard to see that, for each continuous function $u$,

$$
\left\|T_{c, U}^{N, N^{\prime}} u-T_{c, U}^{\infty} u\right\| \leqslant\left\|A_{c}^{N, N^{\prime}}-h_{c}\right\| .
$$

The Proposition follows from (6.1)

(6.4) Proposition. Let $\mathcal{G}_{0}=\mathcal{G}_{c_{0}, u_{0}} \in \mathbb{P}$ be a pseudograph, and let $\epsilon>0$ be fixed. Assume that there exists an open set $U \subset M$ and two compact sets $\mathcal{K} \subset U$ and $\mathcal{K}_{1} \subset M$ such that, for each $x \in \mathcal{K}_{1}$, the minimum in the expression $T_{c_{0}, U}^{\infty} u_{0}(x)=$ $\min _{y \in \bar{U}} u_{0}(y)+h_{c_{0}}(y, x)$ is never reached outside of $\mathcal{K}$. Then there exist integers $N \leqslant N^{\prime}$, a positive number $\delta$ and an open neighborhood $U_{1}$ of $\mathcal{K}_{1}$ such that, for each pseudograph $\mathcal{G} \in \mathbb{P}$ satisfying $\left\|\mathcal{G}-\mathcal{G}_{0}\right\|_{U} \leqslant \delta$, we have

$$
\mathcal{G}_{\mid U} \vdash_{N^{\prime}} \Phi_{U}^{N, N^{\prime}}(\mathcal{G})_{\mid U_{1}}
$$

More precisely, there exists an integer $N_{0}$ and a function $\Lambda: \mathbb{N} \longrightarrow \mathbb{N}$ such that the conclusion holds if $N \geqslant N_{0}$ and $N^{\prime} \geqslant \Lambda(N)$.

Proof. Let us denote by $\partial U$ the boundary of $U$. There exists a positive number $\epsilon$ and a neighborhood $U_{1}$ of $\mathcal{K}_{1}$ such that, for each $x \in \bar{U}_{1}$,

$$
\min _{y \in \partial U} u_{0}(y)+h_{c_{0}}(y, x) \geqslant \min _{y \in U} u_{0}(y)+h_{c_{0}}(y, x)+7 \epsilon .
$$

In view of (6.1), there exist integers $N$ and $N^{\prime}$ such that

$$
\left\|A_{c_{0}}^{N, N^{\prime}}-h_{c_{0}}\right\| \leqslant \epsilon .
$$

For fixed $N$ and $N^{\prime}$, the function $A_{c}^{N, N^{\prime}}$ depends continuously on $c \in H^{1}(M, \mathbb{R})$; see (6.1). As a consequence, if $c$ is sufficiently close to $c_{0}$, we have

$$
\left\|A_{c_{0}}^{N, N^{\prime}}-A_{c}^{N, N^{\prime}}\right\| \leqslant \epsilon \text {. }
$$

For these values of $N$ and $N^{\prime}$, if $u \in C(M, \mathbb{R})$ is such that $\sup _{U}\left|u-u_{0}\right| \leqslant \epsilon$, we have, for each $x \in M$ and $y \in \bar{U}$,

$$
\left|u_{0}(y)+h_{c_{0}}(y, x)-u(y)-A_{c}^{N, N^{\prime}}(y, x)\right| \leqslant 3 \epsilon .
$$

Hence we have the inequality

$$
\min _{y \in \partial U} u(y)+A_{c}^{N, N^{\prime}}(y, x) \geqslant \min _{y \in U} u(y)+A_{c}^{N, N^{\prime}}(y, x)+\epsilon .
$$


As a consequence, if $\left\|\mathcal{G}_{c, u}-\mathcal{G}_{0}\right\|_{U}$ is sufficiently small, then there exists a compact set $\mathcal{K}^{\prime} \subset U$ such that the minimum in the expression

$$
T_{c, U}^{N, N^{\prime}} u(x)=\min _{y \in \bar{U}} u(y)+A_{c}^{N, N^{\prime}}(y, x)
$$

is reached in $\mathcal{K}^{\prime}$ for all $x \in \bar{U}_{1}$. Now let us set $v=T_{c, U}^{N, N^{\prime}} u$ and consider a point

$$
(x, p) \in \overline{\mathcal{G}_{c, v \mid U_{1}}} .
$$

The point $(x, p)$ is the limit of a sequence $\left(x_{n}, p_{n}\right) \in \mathcal{G}_{c, v \mid U_{1}}$. In other words, the points $x_{n} \in U_{1}$ are points of differentiability of $v$, and we have $d v_{x_{n}}+c_{x_{n}}=p_{n}$. Let $y_{n} \in \mathcal{K}^{\prime}$ and $k_{n} \in \mathbb{N}, N \leqslant k_{n} \leqslant N^{\prime}$, satisfy

$$
v\left(x_{n}\right)=u\left(y_{n}\right)+A_{c}\left(0, y_{n} ; k_{n}, x_{n}\right)+k_{n} \alpha(c) .
$$

By extracting a subsequence, we can suppose that the sequence $k_{n}$ is a constant $k$. By arguments similar to those of (2.7) recalling that the function $u$ is semi-concave, we conclude that the function $u$ is differentiable at $y_{n}$, and, setting $z_{n}=c_{y_{n}}+d u_{y_{n}}$, that $\phi^{k}\left(y_{n}, z_{n}\right)=\left(x_{n}, p_{n}\right)$. By extracting another subsequence, we can suppose that the sequence $y_{n}$ has a limit $y \in \mathcal{K}^{\prime}$. We then have

$$
v(x)=u(y)+A_{c}(0, y ; k, x)+k \alpha(c),
$$

so that the function $u$ is differentiable at $y$. Since the function $u$ is semi-concave, we then have $d u_{y}=\lim d u_{y_{n}}$; see Appendix (A.9). At the limit in $\phi^{k}\left(y_{n}, z_{n}\right)=\left(x_{n}, p_{n}\right)$, we get $\phi^{k}(y, z)=(x, p)$, where $z:=d v_{y}+c_{y}$. We have proved that

$$
\overline{\mathcal{G}_{c, v \mid U_{1}}} \subset \bigcup_{k=N}^{N^{\prime}} \phi^{k}\left(\mathcal{G}_{c, u \mid U}\right) .
$$

(6.5) Proposition. Let $c$ and $c^{\prime}$ be two cohomology classes. Assume that, for each weak $K A M$ solution $\mathcal{G}_{0} \in \mathbb{V}_{c}$, there exists a positive number $\epsilon>0$ and an integer $N$ with the following property: For each pseudograph $\mathcal{G} \in \mathbb{P}_{c}$ such that $\left\|\mathcal{G}-\mathcal{G}_{0}\right\| \leqslant \epsilon$, there exists a pseudograph $\mathcal{G}^{\prime} \in \mathbb{P}_{c^{\prime}}$ such that $\mathcal{G} \vdash_{N} \mathcal{G}^{\prime}$. Then $c \vdash c^{\prime}$.

Proof. By compactness of $\mathbb{V}_{c}$, there exists a neighborhood $\mathbb{U}$ of $\mathbb{V}_{c}$ in $\mathbb{P}_{c}$ and an integer $N$ such that, for all $\mathcal{G} \in \mathbb{U}$, we have $\mathcal{G} \vdash_{N} c^{\prime}$. In view of Proposition (6.3), there exist integers $k \leqslant k^{\prime}$ such that $\Phi^{k, k^{\prime}}(\mathcal{G}) \in \mathbb{U}$ for each $\mathcal{G} \in \mathbb{P}_{c}$. We obtain, for each $\mathcal{G} \in \mathbb{P}_{c}$, the existence of a $\mathcal{G}^{\prime} \in \mathbb{P}_{c^{\prime}}$ such that

$$
\mathcal{G} \vdash_{k^{\prime}} \Phi^{k, k^{\prime}}(\mathcal{G}) \vdash_{N} \mathcal{G}^{\prime}
$$

so that $\mathcal{G} \vdash_{k^{\prime}+N} \mathcal{G}^{\prime}$.

\section{Coverings}

As was noticed by Fathi, as well as Contreras and Paternain (see [18 and [1]), it is useful to study the effect of taking finite Galois coverings.

(7.1) Let $P: M_{0} \longrightarrow M$ be a finite connected covering and $P^{*}: H^{1}(M, \mathbb{R}) \longrightarrow$ $H^{1}\left(M_{0}, \mathbb{R}\right)$ be the induced mapping. Let us also denote by $L \circ T P: \mathbb{R} \times T M_{0} \longrightarrow \mathbb{R}$ the lifted Lagrangian

$$
L \circ T P(t, x, v)=L\left(t, P(x), d P_{x}(v)\right),
$$

and by $T^{*} P: T^{*} M_{0} \longrightarrow T^{*} M$ the covering

$$
(x, p) \longmapsto\left(P(x), p \circ d P_{x}^{-1}\right) .
$$


The lifted Hamiltonian $H \circ T^{*} P$ is in natural duality with the Lagrangian $L \circ T P$. As a consequence, the Hamiltonian flow associated to the Lagrangian $L \circ T P$ is the Hamiltonian flow of $H \circ T^{*} P$, which is the lifting of the Hamiltonian flow of $H$. Each overlapping pseudograph $\mathcal{G}=\mathcal{G}_{c, u}$ on $M$ lifts to a pseudograph

$$
P^{*} \mathcal{G}:=T^{*} P^{-1}(\mathcal{G})=\mathcal{G}_{P^{*} c, u \circ P}
$$

on $M_{0}$. Note that $c\left(P^{*} \mathcal{G}\right)=P^{*}(c(\mathcal{G}))$. It is not hard to see that the Aubry set $\tilde{\mathcal{A}}_{L \circ T P}\left(P^{*}(c)\right)$ associated to $L \circ T P$ on $M_{0}$ is precisely

$$
\tilde{\mathcal{A}}_{L \circ T P}\left(P^{*}(c)\right)=T^{*} P^{-1}\left(\tilde{\mathcal{A}}_{L}(c)\right)
$$

while we only have the inclusion

$$
\tilde{\mathcal{N}}_{L \circ T P}\left(P^{*}(c)\right) \supset T^{*} P^{-1}\left(\tilde{\mathcal{N}}_{L}(c)\right)
$$

Finally, if $\mathcal{S}_{L \circ T P}$ is a static class of $\mathcal{A}_{L \circ T P}\left(P^{*}(c)\right)$, then $P\left(\mathcal{S}_{L \circ T P}\right)$ is a static class of $\mathcal{A}_{L}(c)$. Note however that the lifting $P^{-1}\left(\mathcal{S}_{L}\right)$ of a static class of $\mathcal{A}_{L}(c)$ can contain several static classes of $\mathcal{A}_{L \circ T P}\left(P^{*}(c)\right)$. This is illustrated by the following result which, in conjunction with (4.9), allows us to prove the existence of heteroclinic orbits in the case where there is only one static class; see [18 and [1]. We first need another definition. If $\tilde{\mathcal{X}} \subset T^{*} M$ is an invariant set of the time-one flow $\phi$, then we denote by $s \tilde{\mathcal{X}} \subset T^{*} M \times \mathbb{T}$ the set $\bigcup_{t \in \mathbb{R}, x \in \tilde{\mathcal{X}}}\left(\phi_{0}^{t}(x), t\right)$ and by $s \mathcal{X}$ its projection on $M \times \mathbb{T}$.

(7.2) Proposition. Assume that the set $\mathcal{A}(c)$ contains finitely many static classes, and that there exists an open neighborhood $U \subset M \times \mathbb{T}$ of the compact set $s \mathcal{A}(c)$ such that the mapping $h: H_{1}(U, \mathbb{Z}) \longrightarrow H_{1}(M, \mathbb{Z})$ is not surjective, where $h$ is the composition of the mappings

$$
H_{1}(U, \mathbb{Z}) \stackrel{i_{*}}{\longrightarrow} H_{1}(M \times \mathbb{T}, \mathbb{Z}) \stackrel{p_{*}}{\longrightarrow} H_{1}(M, \mathbb{Z})
$$

induced from the inclusion and the projection. Then there exists a finite connected Galois covering $P: M_{0} \longrightarrow M$ with $k$ sheets, $k \geqslant 2$, such that, for each static class $\tilde{\mathcal{S}}$ of $\tilde{\mathcal{A}}(c)$, the lifting $T^{*} P^{-1}(\tilde{\mathcal{S}})$ is the union of exactly $k$ different static classes of $\tilde{\mathcal{A}}_{L \circ T P}\left(P^{*}(c)\right)$.

Proof. Let $N$ be the number of static classes in $\mathcal{A}(c)$. First, we claim that for each static class $\mathcal{S}$, the set $s \mathcal{S}$ is connected. This follows easily from (4.6), As a consequence, we can suppose that the neighborhood $U$ is a union of finitely many connected open sets $U_{i}, 1 \leqslant i \leqslant N$, each of which contains exactly one of the sets $s \mathcal{S}$. Since the group $H^{1}(M, \mathbb{Z})$ is abelian and of finite type, and since the mapping $h: H_{1}(U, \mathbb{Z}) \longrightarrow H_{1}(M, \mathbb{Z})$ is not surjective, there exists an integer $k \geqslant 2$ and a surjective morphism $g: H^{1}(M, \mathbb{Z}) \longrightarrow \mathbb{Z} / k \mathbb{Z}$ whose kernel contains the subgroup $h\left(H_{1}(U, \mathbb{Z})\right)$. There is a connected Galois covering $P: M_{0} \longrightarrow M$ with $k$ sheets associated to this morphism. This means that if $\chi: \pi_{1}(M) \longrightarrow H_{1}(M, \mathbb{Z})$ is the Hurewitz map, then the image $P_{*}\left(\pi_{1}\left(M_{0}\right)\right)$ in $\pi_{1}(M)$ is precisely the kernel of $g \circ \chi$. 
The following diagram commutes:

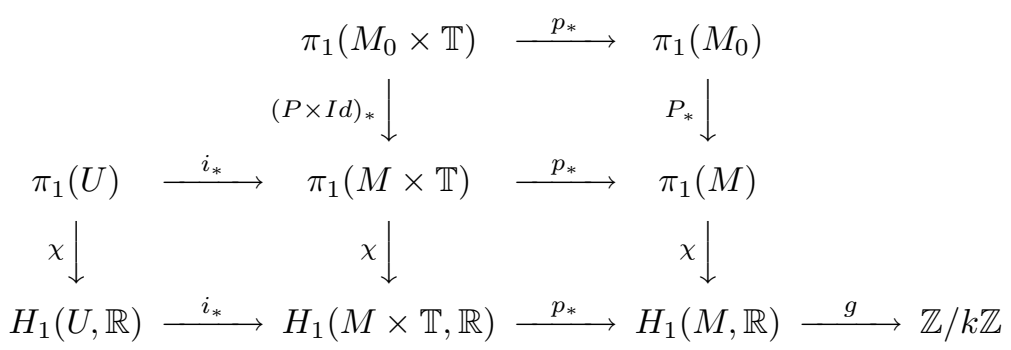

We claim that

$$
i_{*}\left(\pi_{1}(U)\right) \subset(P \times I d)_{*}\left(\pi_{1}\left(M_{0} \times \mathbb{T}\right)\right),
$$

which implies that the covering $P \times I d$ is trivial above $U$. In order to prove the claim, let us first notice that $g \circ \chi \circ p_{*} \circ i_{*}=g \circ p_{*} \circ i_{*} \circ \chi: \pi_{1}(U) \longrightarrow \mathbb{Z} / k \mathbb{Z}$ is the zero map. This implies that the image of the map $i_{*}: \pi_{1}(U) \longrightarrow \pi_{1}(M \times \mathbb{T})$ is contained in the kernel of $g \circ \chi \circ p_{*}$. In order to finish the proof of the claim, we check that the image of $(P \times I d)_{*}$ is precisely the kernel of $g \circ \chi \circ p_{*}$. This follows from the fact $\operatorname{ker}(g \circ \chi)=\operatorname{im}\left(P_{*}\right)$ and from the fact that the upper square of the diagram can be identified with

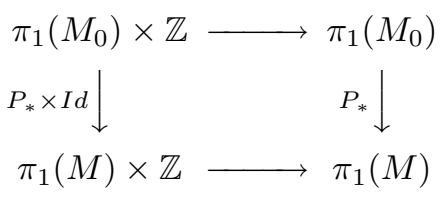

where the horizontal arrows are projections on the first factor. The claim is proved, so that the covering $P \times I d$ is trivial above $U$. It follows that each connected component $U_{i}$ of $U$ has $k$ disjoint connected preimages $V_{i}^{j} \subset M_{0} \times \mathbb{T}$. Now it is not hard to see that the static classes of $\mathcal{A}_{L \circ T P}\left(P^{*}(c)\right)$ are precisely the intersections

$$
T P^{-1}(\mathcal{A}(c)) \cap V_{i}^{j}=\mathcal{A}_{L \circ T P}\left(P^{*}(c)\right) \cap V_{i}^{j}, 1 \leqslant i \leqslant N, 1 \leqslant j \leqslant k .
$$

(7.3) Proposition. Let $P: M_{0} \longrightarrow M$ be a finite Galois covering. Let $c$ and $c^{\prime}$ be two cohomology classes in $H^{1}(M, \mathbb{R})$. If $P^{*}(c) \vdash_{N} P^{*}\left(c^{\prime}\right)$ for the forcing relation associated to the Lagrangian $L \circ T P$ on $M_{0}$, then $c \vdash_{N} c^{\prime}$.

Proof. Let us consider a pseudograph $\mathcal{G} \subset \mathbb{P}_{c}$. If $P^{*}(c) \vdash_{N} P^{*}\left(c^{\prime}\right)$, then there exists a pseudograph $\mathcal{G}^{\prime}$ on $M_{0}$ of cohomology $P^{*}\left(c^{\prime}\right)$ and such that $P^{*} \mathcal{G} \vdash_{N} \mathcal{G}^{\prime}$. Let $\mathbb{D}$ be the group of deck transformations of the covering $P$. The elements of $\mathbb{D}$ are the diffeomorphisms $D$ of $M_{0}$ such that $P \circ D=P$. To each element $D$ of $\mathbb{D}$ we associate the fibered diffeomorphism $T^{*} D$ of $T^{*} M$ defined by

$$
T^{*} D(x, p)=\left(D(x), p \circ d D_{x}^{-1}\right) .
$$

This diffeomorphism is a Deck transformation of the covering $T^{*} P$. Let us prove that there exists a pseudograph $\mathcal{G}^{\prime \prime}$ on $M_{0}$ which is invariant by deck transformations, which has cohomology $P^{*}\left(c^{\prime}\right)$, and is such that $P^{*} \mathcal{G} \vdash_{N} \mathcal{G}^{\prime \prime}$. Let $\eta$ be a form on $M$ with cohomology $c^{\prime}$, and let $P^{*} \eta$ be its lifting to $M_{0}$. We write $\mathcal{G}^{\prime}$ on the form $\mathcal{G}_{P^{*} \eta, u}$. Since the flow of $H \circ T^{*} P$ commutes with Deck transformations, and since the pseudograph $P^{*} \mathcal{G}$ is invariant by deck transformations, we have

$$
P^{*} \mathcal{G}=T^{*} D\left(P^{*} \mathcal{G}\right) \vdash_{N} T^{*} D\left(\mathcal{G}^{\prime}\right)
$$


for each deck transformation $D$. It is easy to check that $T^{*} D\left(\mathcal{G}^{\prime}\right)=\mathcal{G}_{P^{*} \eta, u \circ D^{-1}}$. Setting

$$
v:=\min _{D \in \mathbb{D}} u \circ D^{-1}
$$

and $\mathcal{G}^{\prime \prime}=\mathcal{G}_{\eta, v}$, we have $\mathcal{G}^{\prime \prime} \subset \bigcup_{D \in \mathbb{D}} T^{*} D\left(\mathcal{G}^{\prime}\right)$, hence $P^{*} \mathcal{G} \vdash_{N} \mathcal{G}^{\prime \prime}$, and we have the desired properties for $\mathcal{G}^{\prime \prime}$. Since $P$ is a Galois covering, functions on $M_{0}$ which are invariant by deck transformations are liftings of functions on $M$. As a consequence, there exists a continuous function $w: M \longrightarrow \mathbb{R}$ such that $v=w \circ P$. Hence the pseudograph $\mathcal{G}^{\prime \prime}$ is the lifting of the pseudograph $\mathcal{G}_{\eta, w}$ on $M$. Since $P^{*} \mathcal{G} \vdash_{N} \mathcal{G}^{\prime \prime}=$ $P^{*} \mathcal{G}_{\eta, w}$, we have $\mathcal{G} \vdash_{N} \mathcal{G}_{\eta, w}$. We have asssociated, to each pseudograph $\mathcal{G} \in \mathbb{P}_{c}$, a pseudograph $\mathcal{G}_{\eta, w} \in \mathbb{P}_{c^{\prime}}$ such that $\mathcal{G} \vdash_{N} \mathcal{G}_{\eta, w}$. This proves that $c \vdash_{N} c^{\prime}$.

\section{Mather's mechanism}

We comment and prove Theorem (0.11) Let us first discuss some properties of the subspace $R(c)$ as defined in (0.11).

(8.1) It is useful to consider the Cech cohomology $\check{H}(., \mathbb{R})$ with real coefficients. Recall that $H(., \mathbb{R})$ is the De Rham cohomology. We identify the De Rham cohomology $H^{1}(M, \mathbb{R})$ with the Cech cohomology $\check{H}^{1}(M, \mathbb{R})$. If $K$ is any subset of $M$, we denote by $\check{\imath}_{K}^{*}$ the mapping $\check{H}^{1}(M, \mathbb{R}) \longrightarrow \check{H}^{1}(K, \mathbb{R})$ induced from the inclusion $i_{K}: K \longrightarrow M$. Recalling that the subspace $R(\mathcal{G})$ was defined in $(0.11)$, we have:

Lemma. For each $\mathcal{G} \in \mathbb{V}_{c}$, we have $R(\mathcal{G})=\operatorname{ker}\left(\tilde{\imath}_{\mathcal{I}(\mathcal{G})}^{*}\right)$.

Proof. Consider an open neighborhood $V_{0}$ of $\mathcal{I}(\mathcal{G})$ which is such that, for each open neighborhood $V \subset V_{0}$ of $\mathcal{I}(\mathcal{G})$ in $M, R(\mathcal{G})$ is the set of cohomology classes of smooth closed one-forms which vanish on $V$. Let us fix an open neighborhood $V \subset V_{0}$ of $\mathcal{I}(\mathcal{G})$ in $M$. Clearly, the cohomology classes of smooth closed one-forms which vanish on $V_{0}$ belong to $\operatorname{ker}\left(i_{V}^{*}\right)$ (where $i_{V}^{*}$ is the mapping associated to the inclusion in De Rham cohomology). We have proved the inclusion $R(\mathcal{G}) \subset \operatorname{ker}\left(i_{V}^{*}\right)$. Conversely, let $\omega$ be a smooth closed one-form on $M$ whose De Rham cohomology belongs to $\operatorname{ker}\left(i_{V}^{*}\right)$. This means that the restricted form $\omega_{\mid V}$ is exact. Hence there exists a smooth function $f$ on $V$ such that $\omega_{\mid V}=d f$. There also exists a smooth function $\tilde{f}: M \longrightarrow \mathbb{R}$ which is equal to $f$ in a neighborhood $\tilde{V} \subset V$ of $\mathcal{I}(\mathcal{G})$. The form $\omega-d \tilde{f}$ is a smooth closed form on $M$, cohomologous to $\omega$, and vanishing on $\tilde{V}$. As a consequence, we have $[\omega]=[\omega-d \tilde{f}] \in R(\mathcal{G})$. We have proved that

$$
\operatorname{ker}\left(i_{V}^{*}\right)=R(\mathcal{G})
$$

for all open neighborhoods $V \subset V_{0}$ of $\mathcal{I}(\mathcal{G})$ in $M$. The Lemma follows because $\operatorname{ker}\left(\check{i}_{\mathcal{I}(\mathcal{G})}^{*}\right)$ is equal to $\operatorname{ker}\left(i_{V}^{*}\right)$ when $V$ is a sufficiently small open neighborhood of $\mathcal{I}(\mathcal{G})$ in $M$.

(8.2) In order to avoid confusion, we shall denote by $j_{\tilde{\mathcal{K}}}: \tilde{\mathcal{K}} \longrightarrow T^{*} M$ the inclusion of a subset $\tilde{\mathcal{K}}$ of $T^{*} M$ into $T^{*} M$, and by $\tilde{\jmath}_{\tilde{\mathcal{K}}}^{*}: \check{H}^{1}\left(T^{*} M, \mathbb{R}\right) \longrightarrow \check{H}^{1}(\tilde{\mathcal{K}}, \mathbb{R})$ the associated mapping in Cech cohomology. We identifying the Čech cohomology $\check{H}^{1}\left(T^{*} M, \mathbb{R}\right)$ with the De Rham cohomology $H^{1}\left(T^{*} M, \mathbb{R}\right)$, and the mapping $\check{\pi}^{*}$ : $\check{H}^{1}(M, \mathbb{R}) \longrightarrow \check{H}^{1}\left(T^{*} M, \mathbb{R}\right)$ with $\pi^{*}: H^{1}(M, \mathbb{R}) \longrightarrow H^{1}\left(T^{*} M, \mathbb{R}\right)$.

Lemma. We have $\left(\pi^{*}\right)^{-1}\left(\operatorname{ker}\left(\tilde{\jmath}_{\tilde{\mathcal{N}}(c)}^{*}\right)\right) \subset R(c)$. 
Proof. It is enough to prove that, for each $\mathcal{G} \in \mathbb{V}_{c}$, we have $\operatorname{ker}\left(\check{J}_{\tilde{\mathcal{N}}(c)}^{*}\right) \subset \pi^{*}(R(\mathcal{G}))$. For each $\mathcal{G} \in \mathbb{V}_{c}$, we have $\tilde{\mathcal{I}}(\mathcal{G}) \subset \tilde{\mathcal{N}}(c)$, hence $\operatorname{ker}\left(\tilde{j}_{\tilde{\mathcal{N}}(c)}^{*}\right) \subset \operatorname{ker}\left(\tilde{j}_{\tilde{\mathcal{I}}(\mathcal{G})}^{*}\right)$. So it is enough to prove that the inclusion $\operatorname{ker}\left(\check{J}_{\tilde{\mathcal{I}}(\mathcal{G})}^{*}\right) \subset \pi^{*}(R(\mathcal{G}))$ holds for each $\mathcal{G} \in \mathbb{V}_{c}$. Let us consider such a pseudograph $\mathcal{G}$. Since the projection $\pi: T M \longrightarrow M$ induces a homeomorphism $\pi_{\mid \tilde{\mathcal{I}}(\mathcal{G})}: \tilde{\mathcal{I}}(\mathcal{G}) \longrightarrow \mathcal{I}(\mathcal{G})$, the commutative diagram

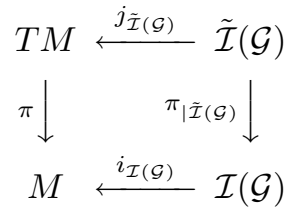

gives rise in Čech cohomology to the diagram

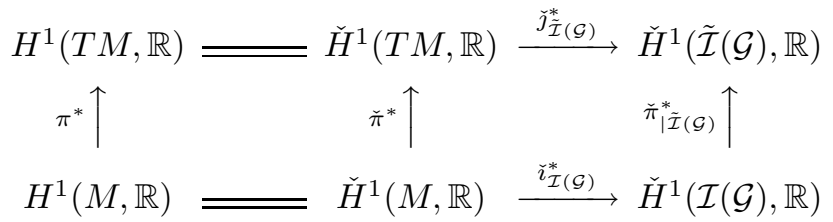

where the vertical arrows are isomorphisms. We conclude that

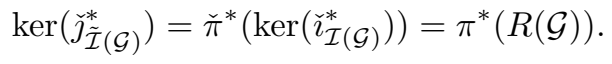

(8.3) Lemma. The space $R(c) \subset H^{1}(M, \mathbb{R})$ depends semi-continuously on $c$ in the following sense: For each $c_{0} \in H^{1}(M, \mathbb{R})$, there exists a neighborhood $V$ of $c_{0}$ in $H^{1}(M, \mathbb{R})$ such that, for each $c \in V$, we have $R\left(c_{0}\right) \subset R(c)$.

Proof. Let us fix a cohomology class $c_{0}$. We claim that, for each $\mathcal{G}_{0} \in \mathbb{V}_{c_{0}}$, there exists $\epsilon_{0}>0$ such that each fixed pseudograph $\mathcal{G}$ of $\mathbb{V}$ which satisfies $\left\|\mathcal{G}-\mathcal{G}_{0}\right\| \leqslant \epsilon_{0}$ has to satisfy

$$
R\left(\mathcal{G}_{0}\right) \subset R(\mathcal{G}) .
$$

This claim follows from the existence of a neighborhood $U$ of $\mathcal{I}\left(\mathcal{G}_{0}\right)$ such that $R\left(\mathcal{G}_{0}\right)$ is the set of cohomology classes of smooth closed one-forms which vanish on $U$, and from the fact that the inclusion $\mathcal{I}(\mathcal{G}) \subset U$ holds when $\mathcal{G}$ is sufficiently close to $\mathcal{G}_{0}$. Let $B_{\mathbb{P}}(\mathcal{G}, \epsilon)$ denote the open ball of center $\mathcal{G}$ and radius $\epsilon$ in $\mathbb{P}$. The compact set $\mathbb{V}_{c_{0}}$ is covered by a finite family of balls $B_{\mathbb{P}}\left(\mathcal{G}_{i}, \epsilon_{i}\right)$ such that $\mathcal{G}_{i} \in \mathbb{V}_{c_{0}}$ and such that $R\left(\mathcal{G}_{i}\right) \subset R(\mathcal{G})$ for each $\mathcal{G} \in B_{\mathbb{P}}\left(\mathcal{G}_{i}, \epsilon_{i}\right)$. Since the map $c$ is continuous and proper on $\mathbb{V}$ (see (3.7)], there exists a $\delta>0$ such that, for $\left|c-c_{0}\right| \leqslant \delta$, we have $\mathbb{V}_{c} \subset \bigcup_{i} B_{\mathbb{P}}\left(\mathcal{G}_{i}, \epsilon_{i}\right)$. As a consequence, if $\mathcal{G}$ belongs to some $\mathbb{V}_{c}$ with $\left|c-c_{0}\right| \leqslant \delta$, then there exists $i$ such that $R\left(c_{0}\right) \subset R\left(\mathcal{G}_{i}\right) \subset R(\mathcal{G})$. We conclude that $R\left(c_{0}\right) \subset R(c)$ for $\left|c-c_{0}\right| \leqslant \delta$.

The following proposition is the main step in the proof of Theorem $(0.11)$. We denote by $B_{E}(r)$ the open ball of radius $r$ centered at the origin in the normed vector space $E$.

(8.4) Proposition. For each $\mathcal{G}_{0} \in \mathbb{V}_{c_{0}}$, there exists a positive number $\epsilon_{0}$ and an integer $N$ such that the following holds: For each pseudograph $\mathcal{G} \in \mathbb{P}$ satisfying $\left\|\mathcal{G}-\mathcal{G}_{0}\right\|<\epsilon_{0}$ and $c(\mathcal{G})-c_{0} \in R\left(c_{0}\right)$, for each cohomology class $c$ satisfying $c-c_{0} \in$ $B_{R\left(c_{0}\right)}\left(\epsilon_{0}\right) \subset R\left(c_{0}\right)$, we have

$$
\mathcal{G} \vdash_{N} c .
$$


Note in this statement that the cohomology class of the pseudograph $\mathcal{G}$ is denoted by $c(\mathcal{G})$, and that the symbol $c$ alone denotes another cohomology class.

(8.5) Proof of Theorem (0.11). We assume the Proposition. For Each $\mathcal{G}_{0} \in$ $\mathbb{V}_{c_{0}}$, we consider the number $\epsilon_{0}$ given by the Proposition, and the open ball $B_{\mathbb{P}}\left(\mathcal{G}_{0}, \epsilon_{0}\right)$ of center $\mathcal{G}_{0}$ and radius $\epsilon_{0}$ in $\mathbb{P}$. Since $\mathbb{V}_{c_{0}}$ is compact, it can be covered by a finite number of these balls; we denote $\mathcal{G}_{i}$ and $\epsilon_{i}$ the associated centers and radii. Since the function $c$ restricted to $\mathbb{V}$ is proper, (3.7), there exists a positive number $\delta$ such that $\mathbb{V}_{c} \subset \bigcup_{i} B_{\mathbb{P}}\left(\mathcal{G}_{i}, \epsilon_{i}\right)$ when $\left|c-c_{0}\right| \leqslant \delta$. Consider two cohomology classes $c$ and $c^{\prime}$ in $c_{0}+B_{R\left(c_{0}\right)}(\epsilon)$, with $\epsilon=\min \left\{\delta, \epsilon_{i}\right\}$. It follows from (6.5) that $c$ forces $c^{\prime}$. The Theorem clearly follows.

(8.6) Proof of the Proposition. Let us fix a $\mathcal{G}_{0} \in \mathbb{V}_{c_{0}}$ and choose a neighborhood $U$ of $\mathcal{I}\left(\mathcal{G}_{0}\right)$ in such a way that $R\left(\mathcal{G}_{0}\right)$ is the set of cohomology classes of smooth closed one-forms vanishing on $U$.

Lemma. There exist $\delta>0$ and $N^{\prime} \leqslant N$ in $\mathbb{N}$ such that, for all overlapping pseudographs $\mathcal{G}$ satisfying $\left\|\mathcal{G}-\mathcal{G}_{0}\right\| \leqslant \delta$, we have

$$
\mathcal{G}_{\mid U} \vdash{ }_{N} \Phi_{U}^{N^{\prime}, N}(\mathcal{G}) .
$$

Proof. Let us write the pseudograph $\mathcal{G}_{0}$ on the form $\mathcal{G}_{c_{0}, u_{0}}$. We have seen in (3.9) that

$$
u_{0}(x)=\min _{y \in M} u_{0}(y)+h_{c_{0}}(y, x)=\min _{y \in \mathcal{A}\left(c_{0}\right)} u_{0}(y)+h_{c_{0}}(y, x) .
$$

As a consequence, we have $T_{U}^{\infty} u_{0}=T_{M}^{\infty} u_{0}=u_{0}$, and the minimum in the definition of $T_{U}^{\infty} u_{0}(x)$ is not reached outside of $\mathcal{I}(\mathcal{G})$, which is a compact set contained in $U$. The Lemma now follows from Proposition (6.4).

(8.7) Lemma. Let us fix a $\delta>0$. There exists $\epsilon_{0}>0$ such that, if we take: on one hand a cohomology class $c$ satisfying $c-c_{0} \in R\left(\mathcal{G}_{0}\right)$ and $\left\|c-c_{0}\right\| \leqslant \epsilon_{0}$ and on the other hand a pseudograph $\mathcal{G} \in \mathbb{P}$ satisfying $\left\|\mathcal{G}-\mathcal{G}_{0}\right\| \leqslant \epsilon_{0}$ and $c(\mathcal{G}) \in c_{0}+R\left(\mathcal{G}_{0}\right)$, then there exists a pseudograph $\mathcal{G}^{\prime} \in \mathbb{P}_{c}$ with the following properties: $\left\|\mathcal{G}^{\prime}-\mathcal{G}_{0}\right\| \leqslant \delta$ and $\mathcal{G}_{\mid U}=\mathcal{G}_{\mid U}^{\prime}$.

Proof. Let us write $\mathcal{G}_{0}=\mathcal{G}_{\eta_{0}, u_{0}}$. In view of the definition of $R\left(\mathcal{G}_{0}\right)$, it is possible to associate to each cohomology class $d \in R\left(\mathcal{G}_{0}\right)$ a closed one-form $\mu_{d}$ which is null on $U$. In addition, we can impose that the correspondence $d \longmapsto \mu_{d}$ is linear. Given a pseudograph $\mathcal{G} \in \mathbb{P}$ and a cohomology $c$ satisfying the hypotheses of the Lemma, we consider the pseudograph

$$
\mathcal{G}^{\prime}=\mathcal{G}+\mathcal{G}_{\mu_{(c-c(\mathcal{G}))}, 0} \in \mathbb{P}_{c} .
$$

It is clear that $\mathcal{G}_{\mid U}^{\prime}=\mathcal{G}_{\mid U}$, that $c\left(\mathcal{G}^{\prime}\right)=c$, and that $\left\|\mathcal{G}^{\prime}-\mathcal{G}_{0}\right\| \leqslant \delta$ if $\epsilon_{0}$ is small enough.

(8.8) We are now in a position to end the proof of the Proposition. Let us consider $\delta$ given by Lemma (8.6), and the associated $\epsilon_{0}$ as given by Lemma (8.7). If $\mathcal{G}$ and $c$ satisfy the hypotheses of the Proposition with this value of $\epsilon_{0}$, then, by Lemma (8.7), there exists a pseudograph $\mathcal{G}^{\prime}$ such that $c\left(\mathcal{G}^{\prime}\right)=c, \mathcal{G}_{\mid U}^{\prime}=\mathcal{G}_{\mid U}$ and $\left\|\mathcal{G}^{\prime}-\mathcal{G}_{0}\right\| \leqslant \delta$. In view of Lemma (8.6), we have $\mathcal{G}_{\mid U} \vdash_{N} \Phi_{U}^{N^{\prime}, N}\left(\mathcal{G}^{\prime}\right)$, so that $\mathcal{G} \vdash_{N} \Phi_{U}^{N^{\prime}, N}\left(\mathcal{G}^{\prime}\right)$. 


\section{Arnold's mechanism for systems with finitely many static classes}

We prove and generalize Theorem $(0.12)$

(9.1) Let $\tilde{\mathcal{H}}_{c}\left(\tilde{\mathcal{S}}, \tilde{\mathcal{S}}^{\prime}\right)$ be the set of orbits of the Mañe set $\tilde{\mathcal{N}}(c)$ which are heteroclinic orbits between the static classes $\tilde{\mathcal{S}}$ and $\tilde{\mathcal{S}}^{\prime}$; we denote by $\mathcal{H}_{c}\left(\mathcal{S}, \mathcal{S}^{\prime}\right)$ its projection on $M$. We have, from Section 4

$$
\tilde{\mathcal{N}}(c)=\tilde{\mathcal{A}}(c) \cup \bigcup_{\mathcal{S}, \mathcal{S}^{\prime}} \tilde{\mathcal{H}}_{c}\left(\mathcal{S}, \mathcal{S}^{\prime}\right),
$$

where the union is taken on all pairs $\left(\mathcal{S}, \mathcal{S}^{\prime}\right)$ of different static classes. In addition, it is useful to recall that

$$
\tilde{\mathcal{H}}_{c}\left(\tilde{\mathcal{S}}, \tilde{\mathcal{S}}^{\prime}\right) \subset E_{c, \mathcal{S}} \tilde{\wedge} \breve{E}_{c, \mathcal{S}^{\prime}}
$$

We say that the set $\tilde{\mathcal{H}}_{c}\left(\mathcal{S}, \mathcal{S}^{\prime}\right)$ is neat if it admits a compact subset $\tilde{\mathcal{K}}$ which contains one and only one point in each orbit of $\phi_{\mid \tilde{\mathcal{H}}_{c}\left(\mathcal{S}, \mathcal{S}^{\prime}\right)}$ and whose projection $\mathcal{K}$ on $M$ is acyclic. This means that $\mathcal{K}$ has a neighborhood $U$ whose inclusion $i$ into $M$ induces the null map $i_{*}: H_{1}(U, \mathbb{R}) \longrightarrow H_{1}(M, \mathbb{R})$.

(9.2) Theorem. Let $c_{0}$ be a cohomology class such that the number of static classes in $\mathcal{A}\left(c_{0}\right)$ is finite and greater than one. Assume in addition that all the sets $\tilde{\mathcal{H}}_{c_{0}}\left(\mathcal{S}, \mathcal{S}^{\prime}\right)$ are neat. Then the class $c_{0}$ is in the interior of its class of $\dashv-$ equivalence.

Let us gather some preliminary consequences of the hypotheses.

(9.3) Lemma. We assume the hypotheses of the Theorem. Let $\mathcal{S}_{0}$ be a static class and $V_{0}$ be a neighborhood of $\mathcal{S}_{0}$.

(i) There exists an open neighborhood $V$ of $\mathcal{S}_{0}$, contained in $V_{0}$, such that the boundary of $V$ does not intersect $\mathcal{I}\left(E_{c_{0}, \mathcal{S}_{0}}\right)$.

(ii) There exists an jacyclic open set $U \subset V_{0}-\mathcal{S}_{0}$ and a static class $\mathcal{S}_{1}$ such that the intersection $U \cap \mathcal{I}\left(E_{c_{0}, \mathcal{S}_{0}}\right)$ is not empty, compact, and contained in $\mathcal{H}\left(\mathcal{S}_{0}, \mathcal{S}_{1}\right)$.

Proof. Let $V_{0}$ be a neighborhood of $\mathcal{S}_{0}$, sufficiently small for Lemma (4.4) to apply, so that we have

$$
V_{0} \cap \mathcal{I}\left(E_{c_{0}, \mathcal{S}_{0}}\right)=\mathcal{S}_{0} \cup \bigcup_{\mathcal{S} \in \mathbb{E}\left(c_{0}\right)-\mathcal{S}_{0}}\left(\mathcal{H}\left(\mathcal{S}_{0}, \mathcal{S}\right) \cap V_{0}\right),
$$

where the union is taken on all static classes $\mathcal{S} \neq \mathcal{S}_{0}$. We shall also assume that $\bar{V}_{0} \cap \mathcal{A}\left(c_{0}\right)=\mathcal{S}_{0}$.

For each static class $\mathcal{S}$, let us consider an acyclic compact set $\tilde{\mathcal{K}}\left(\mathcal{S}_{0}, \mathcal{S}\right)$ which contains one and only one point in every orbit of $\tilde{\mathcal{H}}\left(\mathcal{S}_{0}, \mathcal{S}\right)$, and denote by $\mathcal{K}\left(\mathcal{S}_{0}, \mathcal{S}\right)$ its projection on the base. Clearly, the sets $\tilde{\mathcal{K}}\left(\mathcal{S}_{0}, \mathcal{S}\right), \mathcal{S} \in \mathbb{E}\left(c_{0}\right)-\mathcal{S}_{0}$, are pairwise disjoint and they all belong to the Lipschitz graph $\tilde{\mathcal{I}}\left(E_{c_{0}, \mathcal{S}_{0}}\right)$, so that their projections $\mathcal{K}\left(\mathcal{S}_{0}, \mathcal{S}\right)$ on the base are also pairwise disjoint. Let us consider a static class $\mathcal{S} \neq \mathcal{S}_{0}$. For $n$ large enough, we have $\pi \circ \phi^{-n}\left(\tilde{\mathcal{K}}\left(\mathcal{S}_{0}, \mathcal{S}\right)\right) \subset V_{0}$. In addition, since $\mathcal{K}\left(\mathcal{S}_{0}, \mathcal{S}\right)$ is acyclic in $M$, the compact $\tilde{\mathcal{K}}\left(\mathcal{S}_{0}, \mathcal{S}\right)$ is acyclic in $T^{*} M$. As a consequence, the compact set $\phi^{-n}\left(\tilde{\mathcal{K}}\left(\mathcal{S}_{0}, \mathcal{S}\right)\right)$ is acyclic in $T^{*} M$ and contained in the Lipschitz graph $\tilde{\mathcal{I}}\left(E_{c_{0}, \mathcal{S}_{0}}\right)$, so that $\pi \circ \phi^{-n}\left(\tilde{\mathcal{K}}\left(\mathcal{S}_{0}, \mathcal{S}\right)\right)$ is acyclic in $M$. Consequently, recalling that the number of static classes is finite, there is no loss of generality in supposing that the sets $\mathcal{K}\left(\mathcal{S}_{0}, \mathcal{S}\right), \mathcal{S} \in \mathbb{E}\left(c_{0}\right)-\mathcal{S}_{0}$, are all contained in $V_{0}$. 
Let us prove that each of the sets $\tilde{\mathcal{K}}\left(\mathcal{S}_{0}, \mathcal{S}\right)$ is isolated in $\tilde{\mathcal{I}}\left(E_{c_{0}, \mathcal{S}_{0}}\right)$. Let $F$ be a compact neighborhood of $\mathcal{S}_{0}$ which does not intersect any of the finitely many compact sets $\mathcal{K}\left(\mathcal{S}_{0}, \mathcal{S}\right), \mathcal{S} \in \mathbb{E}\left(c_{0}\right)-\mathcal{S}_{0}$. Since the points of $\tilde{\mathcal{K}}\left(\mathcal{S}_{0}, \mathcal{S}\right)$ are $\alpha$-asymptotic to $\tilde{\mathcal{S}}_{0}$ and $\omega$-asymptotic to $\tilde{\mathcal{S}}$, and since there are finitely many static classes, there exists an integer $N$ such that all the sets $\pi \circ \phi^{n}\left(\tilde{\mathcal{K}}\left(\mathcal{S}_{0}, \mathcal{S}\right)\right), n \in \mathbb{N}, \mathcal{S} \in \mathbb{E}\left(c_{0}\right)-\mathcal{S}_{0}$, are contained in $F$ for $n \leqslant-N$, and do not intersect $\bar{V}_{0}$ for $n \geqslant N$. The set $\left(V_{0}-F\right) \cap \mathcal{I}\left(E_{c_{0}, \mathcal{S}_{0}}\right)$ is thus covered by finitely many pairwise disjoint compact sets of the form $\pi \circ \phi^{n}\left(\tilde{\mathcal{K}}\left(\mathcal{S}_{0}, \mathcal{S}\right)\right), n \in \mathbb{Z}, \mathcal{S} \in \mathbb{E}\left(c_{0}\right)-\mathcal{S}_{0}$. As a consequence, each of the sets $\mathcal{K}\left(\mathcal{S}_{0}, \mathcal{S}\right)$ is isolated in $\left(V_{0}-F\right) \cap \mathcal{I}\left(E_{c_{0}, \mathcal{S}_{0}}\right)$, and then also in $\mathcal{I}\left(E_{c_{0}, \mathcal{S}_{0}}\right)$. Let us fix a static class $\mathcal{S}_{1} \neq \mathcal{S}_{0}$ such that $\mathcal{K}\left(\mathcal{S}_{0}, \mathcal{S}_{1}\right)$ is not empty. Such a static class exists by (4.9) Then, we can find an open neighborhood $U \subset V_{0}$ of $\mathcal{K}\left(\mathcal{S}_{0}, \mathcal{S}_{1}\right)$ such that $U$ is acyclic and such that $U \cap \mathcal{I}\left(E_{c_{0}, \mathcal{S}_{0}}\right)=\mathcal{K}\left(\mathcal{S}_{0}, \mathcal{S}_{1}\right)$ is a non-empty compact set contained in $\mathcal{H}\left(\mathcal{S}_{0}, \mathcal{S}_{1}\right)$. We have proved (ii).

Let us again consider the finite family of pairwise disjoint compact sets $\pi \circ$ $\phi^{n}\left(\tilde{\mathcal{K}}\left(\mathcal{S}_{0}, \mathcal{S}\right)\right), n \in \mathbb{Z},|n| \leqslant N, \mathcal{S} \in \mathbb{E}\left(c_{0}\right)-\mathcal{S}_{0}$. There exists a finite family of pairwise disjoint compact sets $\mathcal{K}_{n}^{\prime}\left(\mathcal{S}_{0}, \mathcal{S}\right), n \in \mathbb{N},|n| \leqslant N, \mathcal{S} \in \mathbb{E}\left(c_{0}\right)-\mathcal{S}_{0}$, such that $\mathcal{K}_{n}^{\prime}\left(\mathcal{S}_{0}, \mathcal{S}\right)$ is a neighborhood of $\pi \circ \phi^{n}\left(\tilde{\mathcal{K}}\left(\mathcal{S}_{0}, \mathcal{S}\right)\right)$. We can clearly assume in addition that the sets $\mathcal{K}_{n}^{\prime}\left(\mathcal{S}_{0}, \mathcal{S}\right)$ do not intersect $\mathcal{S}_{0}$. The set

$$
V=V_{0}-\bigcup_{n \in \mathbb{N},|n| \leqslant N, \mathcal{S} \in \mathbb{E}\left(c_{0}\right)-\mathcal{S}_{0}} \mathcal{K}_{n}^{\prime}\left(\mathcal{S}_{0}, \mathcal{S}\right)
$$

is an open neighborhood of $\mathcal{S}_{0}$ which is contained in $V_{0}$, and its boundary does not intersect $\mathcal{I}\left(E_{c_{0}, \mathcal{S}_{0}}\right)$. We have proved $(i)$.

The following Proposition is the main step in the proof of the Theorem.

(9.4) Proposition. Let $c_{0}$ satisfy the hypotheses of Theorem (9.2), For each weak $K A M$ solution $\mathcal{G}_{0} \in \mathbb{V}_{c_{0}}$, there exists a number $\epsilon>0$ and an integer $N$ such that, if $\mathcal{G} \in \mathbb{P}$ and $c \in H^{1}(M, \mathbb{R})$ satisfy

$$
\left\|\mathcal{G}-\mathcal{G}_{0}\right\| \leqslant \epsilon \text { and }\left|c-c_{0}\right| \leqslant \epsilon,
$$

then $\mathcal{G} \vdash_{N} c$.

(9.5) Proof of the Theorem. We assume the Proposition. Let us cover the compact set $\mathbb{V}_{c_{0}}$ by a finite number of balls $B\left(\mathcal{G}_{i}, \epsilon_{i}\right)$, where $\epsilon_{i}$ is given by the Proposition applied to $\mathcal{G}_{i}$. Since the function $c$ restricted to $\mathbb{V}$ is proper, the union of these finite balls covers the sets $\mathbb{V}_{c}$ for $c$ sufficiently close to $c_{0}$. The Theorem holds by Proposition (6.5).

We now prove the Proposition in three steps.

(9.6) Step 1. Let $\mathcal{G} \in \mathbb{V}_{c_{0}}$ be a fixed point. If there exist only finitely many static classes in $\mathcal{A}\left(c_{0}\right)$, then there exists an elementary solution $E_{0}$ and a neighborhood $U_{0}$ of the corresponding static class $\mathcal{S}_{0}$ such that $\mathcal{G}_{\mid U_{0}}=E_{0 \mid U_{0}}$.

Proof. Let us fix the solution $\mathcal{G}=\mathcal{G}_{c_{0}, u}$. We define a partial order on the set of static classes by saying that $\mathcal{S} \leqslant \mathcal{S}^{\prime}$ if, for each $x \in \mathcal{S}$ and $x^{\prime} \in \mathcal{S}^{\prime}$, we have $h_{c_{0}}\left(x, x^{\prime}\right)=u\left(x^{\prime}\right)-u(x)$. It is easy to check that this relation satisfies the following 
three axioms of order relations:

- $\mathcal{S} \leqslant \mathcal{S}$,

- $\mathcal{S} \leqslant \mathcal{S}^{\prime}$ and $\mathcal{S}^{\prime} \leqslant \mathcal{S}^{\prime \prime} \Longrightarrow \mathcal{S} \leqslant \mathcal{S}^{\prime \prime}$

- $\mathcal{S} \leqslant \mathcal{S}^{\prime}$ and $\mathcal{S}^{\prime} \leqslant \mathcal{S} \Longrightarrow \mathcal{S}=\mathcal{S}^{\prime}$.

Since the number of static classes is finite, there exists an initial element $\mathcal{S}_{0}$, that is, an element which is not greater than any other element. Let us write

$$
u(x)=\min _{a \in \mathcal{A}} u(a)+h_{c_{0}}(a, x)
$$

and consider, for each point $x$, the set $\mathcal{A}\left(c_{0}\right) \cap\left(\mathcal{G} \wedge \breve{E}_{c_{0}, x}\right)$ of points $a$ where the minimum is reached. Let us first assume that $x \in \mathcal{S}_{0}$. In this case, $a$ is a point of minimum if and only if the static class $\mathcal{S}_{a}$ of $a$ satisfies $\mathcal{S}_{a} \leqslant \mathcal{S}_{0}$. Since the class $\mathcal{S}_{0}$ is initial, this implies that $\mathcal{S}_{a}=\mathcal{S}_{0}$, or equivalently, that $a \in \mathcal{S}_{0}$. In other words, for $x \in \mathcal{S}_{0}$, the compact set $\mathcal{A}\left(c_{0}\right) \cap\left(\mathcal{G} \wedge \breve{E}_{c_{0}, x}\right)$ does not intersect static classes other than $\mathcal{S}_{0}$. This implies that, for $x$ sufficiently close to $\mathcal{S}_{0}$, the set $\mathcal{A}\left(c_{0}\right) \cap\left(\mathcal{G} \wedge \breve{E}_{c_{0}, x}\right)$ does not intersect static classes other than $\mathcal{S}_{0}$. Since, for each $x$, the set $\mathcal{A}\left(c_{0}\right) \cap\left(\mathcal{G} \wedge \breve{E}_{c_{0}, x}\right)$ contains a static class, we conclude that, for $x$ sufficiently close to $\mathcal{S}_{0}$, we have

$$
\mathcal{S}_{0}=\mathcal{A}\left(c_{0}\right) \cap\left(\mathcal{G} \wedge \breve{E}_{c_{0}, x}\right) .
$$

As a consequence, we have, if $x$ is sufficiently close to $\mathcal{S}_{0}$,

$$
u(x)=u(a)+h_{c_{0}}(a, x),
$$

for each $a \in \mathcal{S}_{0}$. In other words, the difference $x \longmapsto h_{c_{0}}(a, x)-u(x)$ is the constant $u(a)$ in a neighborhood of $\mathcal{S}_{0}$.

(9.7) STEP 2. Let $\mathcal{S}_{0}$ be a static class of $\mathcal{A}\left(c_{0}\right)$ and let $U_{0}$ be a neighborhood of $\mathcal{S}_{0}$ satisfying $\left(\right.$ ii) of $(9.3)$. There exists a static class $\mathcal{S}_{1}$, an open neighborhood $U_{1}$ of $\mathcal{S}_{1}$ and, for each $\delta>0$, a number $\epsilon>0$ and an integer $N$ with the following property: If $\mathcal{G} \in \mathbb{P}$ satisfies $\left\|\mathcal{G}-E_{c_{0}, \mathcal{S}_{0}}\right\|_{U_{0}} \leqslant \epsilon$ and $c \in H^{1}(M, \mathbb{R})$ satisfies $\left|c-c_{0}\right| \leqslant \epsilon$, then there exists a pseudograph $\mathcal{G}^{\prime} \in \mathbb{P}_{c}$ such that $\left\|\mathcal{G}^{\prime}-E_{c_{0}, \mathcal{S}_{1}}\right\|_{U_{1}} \leqslant \delta$ and

$$
\mathcal{G}_{\mid U_{0}} \vdash_{N} \mathcal{G}_{\mid U_{1}}^{\prime} .
$$

Proof. There exists a static class $\mathcal{S}_{1}$ and an acyclic open set $U \subset U_{0}-\mathcal{A}\left(c_{0}\right)$ such that

$$
\mathcal{I}\left(E_{c_{0}, \mathcal{S}_{0}}\right) \cap \bar{U}=\mathcal{I}\left(E_{c_{0}, \mathcal{S}_{0}}\right) \cap U
$$

is a compact set $\mathcal{K} \subset \mathcal{H}\left(\mathcal{S}, \mathcal{S}_{1}\right)$. Let us fix a point $x_{0} \in \mathcal{S}_{0}$, and denote by $u_{0}$ the function $h_{c_{0}}\left(x_{0},.\right)$.

(9.8) Lemma. There exists a neighborhood $U_{1}$ of $\mathcal{S}_{1}$ such that the equality

$$
T_{c_{0}, U}^{\infty} u_{0}(y)=h_{c_{0}}\left(x_{0}, x_{1}\right)+h_{c_{0}}\left(x_{1}, y\right)=h_{c_{0}}\left(x_{0}, x\right)+h_{c_{0}}\left(x, x_{1}\right)+h_{c_{0}}\left(x_{1}, y\right)
$$

holds for all $x \in \mathcal{K}, y \in U_{1}$, and $x_{1} \in \mathcal{S}_{1}$. As a consequence, we have

$$
\Phi_{U}^{\infty}\left(E_{c_{0}, \mathcal{S}_{0}}\right)_{\mid U_{1}}=E_{c_{0}, \mathcal{S}_{1} \mid U_{1}},
$$

and the minimum in the definition of $T_{c_{0}, U}^{\infty} u_{0}(y)$ is not reached outside of $\mathcal{K}$ when $y \in U_{1}$. 
Proof. Let us set $v=T_{c_{0}, U}^{\infty} u_{0}$ for simplicity. Recall, from (3.9), that all weak KAM solutions $v \in \mathbb{V}_{c_{0}}$ satisfy $v(y)=\min _{a \in \mathcal{A}\left(c_{0}\right)} v(a)+h_{c_{0}}(a, y)$. Here, we obtain

$$
v(y)=\min _{x \in \bar{U}, a \in \mathcal{A}\left(c_{0}\right)} h_{c_{0}}\left(x_{0}, x\right)+h_{c_{0}}(x, a)+h_{c_{0}}(a, y) .
$$

We claim that, for $y \in \mathcal{S}_{1}$, the set of minimizing pairs $(x, a)$ is $\mathcal{K} \times \mathcal{S}_{1}$. Indeed, if $(x, a) \in \mathcal{K} \times \mathcal{S}_{1}$, then $x \in E_{c_{0}, x_{0}} \wedge \breve{E}_{c_{0}, a}$, so that $h_{c_{0}}\left(x_{0}, x\right)+h_{c_{0}}(x, a)=h_{c_{0}}\left(x_{0}, a\right)$, and

$$
\begin{aligned}
& h_{c_{0}}\left(x_{0}, x\right)+h_{c_{0}}(x, a)+h_{c_{0}}(a, y) \\
& \quad=h_{c_{0}}\left(x_{0}, y\right)=\min _{\left(z, z^{\prime}\right) \in M \times M} h_{c_{0}}\left(x_{0}, z\right)+h_{c_{0}}\left(z, z^{\prime}\right)+h_{c_{0}}\left(z^{\prime}, y\right) .
\end{aligned}
$$

Hence we have

$$
h_{c_{0}}\left(x_{0}, x\right)+h_{c_{0}}(x, a)+h_{c_{0}}(a, y)=\min _{\left(z, z^{\prime}\right) \in \bar{U} \times \mathcal{A}(c)} h_{c_{0}}\left(x_{0}, z\right)+h_{c_{0}}\left(z, z^{\prime}\right)+h_{c_{0}}\left(z^{\prime}, y\right) .
$$

We have proved that the pairs of $\mathcal{K} \times \mathcal{S}_{1}$ are minimizing in the equation $(\diamond)$ for $y \in \mathcal{S}_{1}$.

Let us now prove that they are the only minimizing pairs. A pair $(x, a)$ is minimizing if and only if $h_{c_{0}}\left(x_{0}, a\right)+h_{c_{0}}(a, y)=h_{c_{0}}\left(x_{0}, y\right)$ and $h_{c_{0}}\left(x_{0}, x\right)+h_{c_{0}}(x, a)=$ $h_{c_{0}}\left(x_{0}, a\right)$. The second equality implies

$$
x \in E_{c_{0}, \mathcal{S}_{0}} \wedge \breve{E}_{c_{0}, \mathcal{S}(a)} \subset \mathcal{I}\left(E_{c_{0}, \mathcal{S}_{0}}\right) .
$$

Since $\mathcal{I}\left(E_{c_{0}, \mathcal{S}_{0}}\right) \cap \bar{U}=\mathcal{K}$, this implies $x \in \mathcal{K}$. If $x \in \mathcal{K}$ and $a \in \mathcal{A}\left(c_{0}\right)$, then the equality $h_{c_{0}}(x, a)=h_{c_{0}}(x, y)+h_{c_{0}}(y, a)$ holds for all $y \in \mathcal{S}_{1}$. Indeed, let $x(n)=\pi \circ \phi^{n}\left(x,-\partial_{1} h(x, a)\right)$ be the projection of the orbit of the only point of $\breve{E}_{c_{0}, \mathcal{S}(a)}$ above $x$. We have, for each $n \in \mathbb{N}$, the equality of calibration by $-h_{c_{0}}(., a)$ :

$$
A_{c_{0}}(0, x, n, x(n))+n \alpha\left(c_{0}\right)=h_{c_{0}}(x, a)-h_{c_{0}}(x(n), a) .
$$

Let $n_{k}$ be an increasing sequence of integers such that the subsequence $x\left(n_{k}\right)$ has a limit $\omega \in \mathcal{S}_{1}$. Taking the liminf as $k \longrightarrow \infty$, we get $h_{c_{0}}(x, \omega) \leqslant h_{c_{0}}(x, a)-h_{c_{0}}(\omega, a)$, which implies the desired equality for $\omega$, and then for all points of $\mathcal{S}_{1}$.

Since $(x, a)$ is a minimizing pair for $v(y)$, we get, by decomposing $h_{c_{0}}(x, a)$ in the expression of $v$,

$$
v(y)=h_{c_{0}}\left(x_{0}, x\right)+h_{c_{0}}(x, y)+h_{c_{0}}(y, a)+h_{c_{0}}(a, y),
$$

and, since $v(y) \leqslant h_{c_{0}}\left(x_{0}, x\right)+h_{c_{0}}(x, y)$, we finally obtain that $h_{c_{0}}(y, a)+h_{c_{0}}(a, y) \leqslant$ 0 so that $a \in \mathcal{S}_{1}$. We have proved the claim. In addition, we have proved, for $x_{1} \in \mathcal{S}_{1}$ and $x \in \mathcal{K}$, the equality

$$
v\left(x_{1}\right)=h_{c_{0}}\left(x_{0}, x\right)+h_{c_{0}}\left(x, x_{1}\right)=h_{c_{0}}\left(x_{0}, x_{1}\right) .
$$

As a consequence, for $y \in \mathcal{S}_{1}$, each point $a \in \mathcal{A}(c)$ which is minimizing in the equation

$$
v(y)=\min _{a \in \mathcal{A}\left(c_{0}\right)} v(a)+h_{c_{0}}(a, y)
$$

belongs to $\mathcal{S}_{1}$. Since $\mathcal{S}_{1}$ is isolated in $\mathcal{A}(c)$, the conclusion also holds for $y$ sufficiently close to $\mathcal{S}_{1}$. We then have the equality

$$
v(y)=v\left(x_{1}\right)+h_{c_{0}}\left(x_{1}, y\right)=h_{c_{0}}\left(x_{0}, x\right)+h_{c_{0}}\left(x, x_{1}\right)+h_{c_{0}}\left(x_{1}, y\right)
$$

for all $x_{1} \in \mathcal{S}_{1}$ and $x \in \mathcal{K}$ (and no other $x$ in $\bar{U}$ ). 
(9.9) Applying (6.4), we get the existence of a positive $\epsilon^{\prime}$ and of integers $N \leqslant N^{\prime}$ such that each $\mathcal{G} \in \mathbb{P}$ which satisfies $\left\|\mathcal{G}-E_{c_{0}, \mathcal{S}_{0}}\right\|_{U} \leqslant \epsilon^{\prime}$ also satisfies

$$
\mathcal{G}_{\mid U} \vdash_{N^{\prime}} \Phi_{U}^{N, N^{\prime}}(\mathcal{G})_{\mid U_{1}} .
$$

Since $\Phi_{U}^{\infty}\left(E_{c_{0}, \mathcal{S}_{0}}\right)_{\mid U_{1}}=E_{c_{0}, \mathcal{S}_{1} \mid U_{1}}$ in view of the Lemma, and by $(6.3)$, the integers $N$ and $N^{\prime}$ can be chosen such that, in addition, we have

$$
\left\|\Phi_{U}^{N, N^{\prime}}\left(E_{c_{0}, \mathcal{S}_{0}}\right)-E_{c_{0}, \mathcal{S}_{1}}\right\|_{U_{1}} \leqslant \delta / 2 .
$$

Reducing $\epsilon^{\prime}$ if necessary, we can furthermore assume, by continuity of $\Phi_{U}^{N, N^{\prime}}$, that

$$
\left\|\Phi_{U}^{N, N^{\prime}}(\mathcal{G})-\Phi_{U}^{N, N^{\prime}}\left(E_{c_{0}, \mathcal{S}_{0}}\right)\right\|_{U_{1}} \leqslant \delta / 2
$$

when $\left\|\mathcal{G}-E_{c_{0}, \mathcal{S}_{0}}\right\|_{U} \leqslant \epsilon^{\prime}$, so that

$$
\left\|\Phi_{U}^{N, N^{\prime}}(\mathcal{G})-E_{c_{0}, \mathcal{S}_{1}}\right\|_{U_{1}} \leqslant \delta .
$$

Since $U$ is acyclic, for each cohomology $c$ and each pseudograph $\mathcal{G}$, there exists a pseudograph $\mathcal{G}(c)$ which has cohomology $c$ and such that $\mathcal{G}_{\mid U}=\mathcal{G}(c)_{\mid U}$. There exists a positive $\epsilon$ such that, if $\left|c-c_{0}\right| \leqslant \epsilon$ and if $\left\|\mathcal{G}-E_{c_{0}, \mathcal{S}_{0}}\right\|_{U} \leqslant \epsilon$, then we have

$$
\left\|\mathcal{G}(c)-E_{c_{0}, \mathcal{S}_{0}}\right\|_{U} \leqslant \epsilon^{\prime} .
$$

Note that this norm does not depend on the choice of $\mathcal{G}(c)$. As a consequence, setting $\mathcal{G}^{\prime}=\Phi_{U}^{N, N^{\prime}}(\mathcal{G}(c))$, we have $c\left(\mathcal{G}^{\prime}\right)=c$,

$$
\mathcal{G}_{\mid U}=\mathcal{G}(c)_{\mid U} \vdash_{N^{\prime}} \mathcal{G}_{\mid U_{1}}^{\prime},
$$

and

$$
\left\|\mathcal{G}^{\prime}-E_{c_{0}, \mathcal{S}_{1}}\right\|_{U_{1}} \leqslant \delta
$$

(9.10) SteP 3. Let $\mathcal{S}_{1}$ be a static class in $\mathcal{A}\left(c_{0}\right)$ satisfying $(i)$ of (9.3), and let $U_{1}$ be a fixed neighborhood of $\mathcal{S}_{1}$. There exist a number $\delta>0$ and an integer $N$ such that, if $\mathcal{G}^{\prime} \in \mathbb{P}$ satisfies $\left\|\mathcal{G}^{\prime}-E_{c_{0}, \mathcal{S}_{1}}\right\|_{U_{1}} \leqslant \delta$, then $\mathcal{G}_{\mid U_{1}}^{\prime} \vdash_{N} c\left(\mathcal{G}^{\prime}\right)$.

Proof. There exists an open neighborhood $V_{1} \subset U_{1}$ of $\mathcal{S}_{1}$ such that $\mathcal{I}\left(E_{c_{0}, \mathcal{S}_{1}}\right) \cap$ $V_{1}=\mathcal{I}\left(E_{c_{0}, \mathcal{S}_{1}}\right) \cap \bar{V}_{1}$. (This is $(i)$ of $(9.3)$, ) Let $x_{1}$ be a point of $\mathcal{S}_{1}$ and set $u_{1}=h_{c_{0}}\left(x_{1},.\right)$. Recall that (by definition)

$$
T_{c_{0}, V_{1}}^{\infty} u_{1}(x)=\min _{y \in \bar{V}_{1}} h_{c_{0}}\left(x_{1}, y\right)+h_{c_{0}}(y, x) .
$$

Taking $y=x_{1}$ in this expression, we obtain the inequality $T_{c_{0}, V_{1}}^{\infty} u_{1}(x) \leqslant u_{1}(x)$. On the other hand, we have the triangle inequality $u_{1}(x) \leqslant h_{c_{0}}\left(x_{1}, y\right)+h_{c_{0}}(y, x)$ for each $y$, so that $T_{c_{0}, V_{1}}^{\infty} u_{1}(x)=u_{1}(x)$ and

$$
\min _{y \in \bar{V}_{1}} h_{c_{0}}\left(x_{1}, y\right)+h_{c_{0}}(y, x)=h_{c_{0}}\left(x_{1}, x\right)=\min _{y \in M} h_{c_{0}}\left(x_{1}, y\right)+h_{c_{0}}(y, x) .
$$

By (3.6) the points $y$, where this last minimum is reached, belong to $\mathcal{I}\left(E_{c_{0}, \mathcal{S}_{1}}\right)$. As a consequence, for each $x \in M$, the points where the minimum is reached in the definition of $T_{c_{0}, V_{1}}^{\infty} u_{1}(x)$ belong to $\mathcal{I}\left(E_{c_{0}, \mathcal{S}_{1}}\right) \cap V_{1}$, which is a compact set contained in $V_{1}$. In view of (6.4), there exist integers $N$ and $N^{\prime}$ and a positive real number $\delta$ such that, if $\mathcal{G}^{\prime} \in \mathbb{P}$ satisfies $\left\|\mathcal{G}^{\prime}-E_{c_{0}, \mathcal{S}_{1}}\right\|_{V_{1}} \leqslant \delta$, then

$$
\mathcal{G}_{\mid V_{1}}^{\prime} \vdash_{N} \Phi_{V_{1}}^{N, N^{\prime}}\left(\mathcal{G}^{\prime}\right) \text {. }
$$

STEP 3

The Proposition obviously follows from the three steps above. 


\section{Applications}

\section{Twist maps}

The case where $M=\mathbb{T}$ is well known and has been studied many times. The resulting time-one flow is then a finite composition of right twist maps of the biinfinite annulus $T^{*} \mathbb{T}$. In view of $(0.10)$, much of what is known on the existence of orbits with prescribed behavior is summed up in the following discussion.

(10.1) Let $G \in H^{1}(\mathbb{T}, \mathbb{R})$ be the set of cohomology classes of invariant curves which are Lipschitz graphs. The set $G$ is closed, and every point $c \in G$ is alone in its class of $\dashv \vdash$-equivalence, as follows from (5.2). Conversely, if $c$ does not belong to $G$, then all the sets $\mathcal{I}(\mathcal{G}), \mathcal{G} \in \mathbb{V}_{c}$, are properly contained in $\mathbb{T}$. It follows that $R(\mathcal{G})=\mathbb{R}$ for each $\mathcal{G} \in \mathbb{V}_{c}$, so that $R(c)=\mathbb{R}$ and, in view of (0.11) $c$ is in the interior of its class of equivalence. The classes of $\dashv \Vdash$-equivalence are the points of $G$ and the connected components of the complement of $G$.

(10.2) For completeness, we recall without proof some of the special properties of Aubry sets in dimension one; see 14 for example. The function $\alpha$ is differentiable, and its differential $\alpha^{\prime}(c)$ is the rotation number of every orbit of $\tilde{\mathcal{N}}(c)$. If $\alpha^{\prime}(c)$ is irrational, then there is only one element in $\mathbb{V}_{c}$. If $\alpha^{\prime}(c)$ is rational, then the Mather set $\tilde{\mathcal{M}}(c)$ is made of periodic orbits.

\section{Generalized Arnold example}

(11.1) In this application, we take

$$
M=\mathbb{T} \times N,
$$

where $N$ is a compact manifold of dimension $d-1$, and denote by $q=\left(q_{1}, q_{2}\right)$ the points of $M$. We assume that the homology group $H_{1}(N, \mathbb{Z})$ is not trivial. We denote the points of $T M$ by $(q, v)=\left(q_{1}, q_{2}, v_{1}, v_{2}\right)$, where $\left(q_{1}, v_{1}\right) \in T \mathbb{T}$ and $\left(q_{2}, v_{2}\right) \in T N$. In the same way, we denote by $(q, p)=\left(q_{1}, q_{2}, p_{1}, p_{2}\right)$ the points of $T^{*} M$. We will consider the projection $\pi_{1}: \mathbb{T} \times N \longrightarrow \mathbb{T}$ and the induced mapping

$$
\pi_{1}^{*}: H^{1}(\mathbb{T}, \mathbb{R}) \longrightarrow H^{1}(\mathbb{T} \times N, \mathbb{R}) .
$$

(11.2) Let us fix a point 0 in $N$. We will consider Lagrangian systems which satisfy

$$
L\left(t, q_{1}, q_{2}, v_{1}, v_{2}\right)>L\left(t, q_{1}, 0, v_{1}, 0\right)
$$

for all $\left(q_{2}, v_{2}\right) \neq(0,0)$, all $t \in \mathbb{R}$ and all $\left(q_{1}, v_{1}\right) \in T \mathbb{T}$. Let $\partial_{v} L: T M \longrightarrow \mathbb{T}^{*} M$ be the Legendre transform associated to $L$. We denote by $\mathbb{T}_{1}$ the submanifold $\mathbb{T} \times\{0\}$ of $M$, by $T^{*} \mathbb{T}_{1}$ the submanifold $\left\{q_{2}=0, p_{2}=0\right\}$ of $T^{*} M$, and $T \mathbb{T}_{1}$ the submanifold $\left\{q_{2}=0, v_{2}=0\right\}$ of $T M$. We have

$$
\partial_{v} L\left(T \mathbb{T}_{1}\right)=T^{*} \mathbb{T}_{1},
$$

and this manifold is invariant under the Hamiltonian flow. Moreover, the restriction of the flow to $T^{*} \mathbb{T}_{1}$ is the Hamiltonian flow of the restriction $H_{1}\left(t, q_{1}, p_{1}\right):=$ $H\left(t, q_{1}, p_{1}, 0,0\right)$ of $H$. Setting $L_{1}\left(t, q_{1}, v_{1}\right)=L\left(t, q_{1}, 0, v_{1}, 0\right)$, we see that $L_{1}$ is the Lagrangian associated to $H_{1}$. We denote by $\phi_{1}$ the restriction of $\phi$ to $T^{*} \mathbb{T}$.

(11.3) Theorem. Under the non-degeneracy conditions (11.4) and (11.5) to be specified below, the image of $\pi_{1}^{*}$ is contained in one class of $-\Vdash$-equivalence. 
(11.4) GENERICITY PROPERTY FOR $\phi_{1}$. We assume that every rotational invariant circle of $\phi_{1}$ which contains a periodic orbit is completely periodic (every orbit of this circle is periodic). We could, more simply, require that the map $\phi_{1}$ does not have any invariant circle containing a periodic orbit. This property is known to be generic in any reasonable sense of the term. However, allowing periodic circles includes the important case where $\phi_{1}$ is integrable, as in the original Arnold's example.

(11.5) Non-DEgEnERACY OF EXTERnAl Homoclinics. We assume that, for each $c \in \pi_{1}^{*}\left(H^{1}(\mathbb{T}, \mathbb{R})\right)$, there exists a finite Galois covering $P: M_{0} \longrightarrow M$ such that the set

$$
\tilde{\mathcal{N}}_{L \circ T P}\left(P^{*}(c)\right)-T^{*} P^{-1}\left(T^{*} \mathbb{T}_{1}\right)
$$

is not empty and contains finitely many orbits. Note that, since $H^{1}(N, \mathbb{Z})$ is not zero, it follows from (7.2), (4.5) and (11.6) below that there exists a finite Galois covering $P: M_{0} \longrightarrow M$ such that the set under consideration is not empty. So the important point of our assumption is finiteness. As the reader will see in the proof, this assumption could be somewhat weakened.

(11.6) Lemma. For each cohomology $c=\pi_{1}^{*}\left(c_{1}\right)$ with $c_{1} \in H^{1}(\mathbb{T}, \mathbb{R})$, we have $\mathcal{N}(c) \subset \mathbb{T}_{1}$. As a consequence, the restriction to $\mathbb{T}_{1}$ gives a bijection between the set $\mathbb{V}_{c}$ and the set $\mathbb{V}_{c_{1}}$ associated to the Lagrangian $L_{1}$ on $T \mathbb{T}_{1}$.

Proof. Let us fix a cohomology $c_{1} \in H^{1}(\mathbb{T}, \mathbb{R})$ and its image $c:=\pi_{1}^{*}\left(c_{1}\right)$. Let $\mu$ be a form on $\mathbb{T}$ which represents $c_{1}$ and $\eta$ be its pull back on $M=\mathbb{T} \times N$. Consider a pseudograph $\mathcal{G} \in \mathbb{V}_{c}$ and write it $\mathcal{G}=\mathcal{G}_{\eta, u}$. We want to prove that $\tilde{\mathcal{I}}(\mathcal{G}) \subset T^{*} \mathbb{T}_{1}$. Let $(q(t), p(t))$ be the trajectory of the Hamiltonian flow starting in $\tilde{\mathcal{I}}(\mathcal{G})$. We have, for $k<l$ in $\mathbb{Z}$,

$$
u(q(l))-u(q(k))=\int_{k}^{l} L(\sigma, q(\sigma), \dot{q}(\sigma))-\mu_{q_{1}(\sigma)}\left(\dot{q}_{1}(\sigma)\right)+\alpha(c) d \sigma
$$

and

$$
u\left(q_{1}(t), 0\right)-u\left(q_{1}(s), 0\right) \leqslant \int_{k}^{l} L\left(\sigma,\left(q_{1}(\sigma), 0, \dot{q}_{1}(\sigma), 0\right)\right)-\mu_{q_{1}(\sigma)}\left(\dot{q}_{1}(\sigma)\right)+\alpha(c) d \sigma .
$$

It follows that

$$
\int_{k}^{l} L(\sigma, q(\sigma), \dot{q}(\sigma))-L\left(\sigma,\left(q_{1}(\sigma), 0\right),\left(\dot{q}_{1}(\sigma), 0\right)\right) d \sigma \leqslant 2(\max u-\min u) .
$$

Let us denote by $\tilde{L}$ the function

$$
\tilde{L}(t, q, v)=L(t, q, v)-L\left(t,\left(q_{1}, 0\right),\left(v_{1}, 0\right)\right)
$$

which is positive except on $T \mathbb{T}_{1}$. Since the integral $\int_{\mathbb{R}} \tilde{L}(\sigma, q(\sigma), \dot{q}(\sigma)) d \sigma$ is finite, we have

$$
\liminf _{|\sigma| \longrightarrow \infty} \tilde{L}(\sigma, q(\sigma), \dot{q}(\sigma))=0
$$

and consequently $\liminf |\sigma| \longrightarrow \infty\left(q_{2}(t), v_{2}(t)\right)=0$. We now return to the inequality

$$
\int_{k}^{l} \tilde{L}(\sigma, q(\sigma), \dot{q}(\sigma)) d \sigma \leqslant u(q(t))-u\left(q_{1}(t), 0\right)-u(q(s))+u\left(q_{1}(s), 0\right)
$$


from which we get

$$
\int_{-\infty}^{\infty} \tilde{L}(\sigma, q(\sigma), \dot{q}(\sigma)) d \sigma=0,
$$

which implies that $\left(q_{2}, v_{2}\right) \equiv 0$. We have proved that $\tilde{\mathcal{I}}(\mathcal{G}) \subset T^{*} \mathbb{T}_{1}$.

(11.7) Let us fix cohomologies $c=\pi_{1}^{*}\left(c_{1}\right), c_{1} \in H^{1}(\mathbb{T}, \mathbb{R})$, such that there exists an invariant Lipschitz graph $\mathcal{G}$ in $\mathbb{V}_{c_{1}}$. If the rotation number of $\phi_{1 \mid \mathcal{G}}$ is irrational, then $\mathbb{V}_{c_{1}}$ contains only one element. As a consequence, $\mathbb{V}_{c}$ also contains only one element, so that $\tilde{\mathcal{N}}(c)=\tilde{\mathcal{A}}(c)=\mathcal{G}$, and there is only one static class in $\tilde{\mathcal{A}}(c)$. If the rotation number is rational, then in view of (11.4) the graph $\mathcal{G}$ is a union of periodic orbits, so that $\mathcal{G}=\tilde{\mathcal{M}}(c)$. As a consequence, we have $\mathcal{A}(c)=\mathbb{T}_{1}$, and there is only one static class.

In view of (11.5), there exists a finite Galois covering $P: M_{0} \longrightarrow M$ such that the Lagrangian $L \circ T P$ satisfies the hypotheses of (9.2). As a consequence, the cohomology $P^{*}(c)$ is in the interior of its forcing class for $L \circ T P$. It follows from (7.3) that the cohomology $c$ is in the interior of its forcing class for $L$.

(11.8) Let $c=\pi_{1}^{*}\left(c_{1}\right)$ be such that each set $\mathcal{I}(\mathcal{G}), \mathcal{G} \in \mathbb{V}_{c}$, is properly contained in $\mathbb{T}_{1}$. Applying (0.11), we observe that $R(c)=H^{1}(M, \mathbb{R})$, and $c$ is in the interior of its forcing class.

(11.9) We have proved that each $c \in \pi_{1}^{*}(\mathbb{T}, \mathbb{R})$ is in the interior of its forcing class. Since the subspace $\pi_{1}^{*}\left(H^{1}(\mathbb{T}, \mathbb{R})\right)$ is obviously connected, it is contained in one forcing class.

\section{APPENDIX}

\section{A Semi-concave functions}

We recall some useful facts on semi-concave functions. In all this section, $M$ is a compact manifold of dimension $d$. It is useful for the sequel to fix once and for all a finite atlas $\Phi$ of $M$ composed of charts $\varphi: B_{3} \longrightarrow M$, where $B_{r}$ is the open ball of radius $r$ centered at zero in $\mathbb{R}^{d}$. We assume that the sets $\varphi\left(B_{1}\right), \varphi \in \Phi$, cover $M$. A family $F$ of $C^{2}$ functions is said bounded if there exists a constant $C>0$ such that

$$
\left\|d^{2}(u \circ \varphi)_{x}\right\| \leqslant C
$$

for all $x \in B_{1}, \varphi \in \Phi$, and $u \in F$. Note that a bounded family is not required to be bounded in $C^{0}$ norm, but will automatically be bounded in $C^{1}$ norm and thus equi-Lipschitz. The notion of a bounded family of functions does not depend on the atlas $\Phi$.

(A.1) A function $u: M \longrightarrow \mathbb{R}$ is called semi-concave if there exists a bounded subset $F_{u}$ of the set $C^{2}(M, \mathbb{R})$ such that

$$
u=\inf _{f \in F_{u}} f .
$$

A family $\mathbb{U}$ of functions is called equi-semi-concave if there exists a bounded set $F$ of functions in $C^{2}(M, \mathbb{R})$, and, for each function $u \in \mathbb{U}$, a subset $F_{u}$ of $F$ such that

$$
u=\inf _{f \in F_{u}} f .
$$

Let us first collect some easy consequences of this definition. We shall prove later that the infima could be replaced by minima in these definitions. 
(A.2) An equi-semi-concave set of functions is equi-Lipschitz.

(A.3) If $\mathbb{U}$ is an equi-semi-concave set of functions on $M$, and if the infimum $\inf _{u \in \mathbb{U}} u\left(x_{0}\right)$ is finite for some $x_{0} \in M$, then the function $v(x)=\inf _{u \in \mathbb{U}} u(x)$ is finite and semi-concave.

(A.4) Let $\mathbb{U}$ be an equi-semi-concave set of functions on $N \times M$, where $N$ is another compact manifold. Then the functions $u(x,):. M \longrightarrow \mathbb{R}, x \in N, u \in \mathbb{U}$, form an equi-semi-concave set.

(A.5) We say that the linear form $p \in T_{x} M$ is a proximal super-differential of the function $u$ at point $x$ if there exists a $C^{2}$ function $f$ such that $f-u$ has a minimum at $x$ and $d f_{x}=p$. The definition would not be changed by requiring that the function $f$ is smooth and that the minimum is strict. We say that a linear form $p \in T_{x} M$ is a $K$-super-differential of the function $u$ at point $x$ if for each chart $\varphi \in \Phi$ and each point $y \in B_{2}$ satisfying $\varphi(y)=x$, the inequality

$$
u \circ \varphi(z)-u \circ \varphi(y) \leqslant p \circ d \varphi_{y}(z-y)+K\|z-y\|^{2}
$$

holds for each $z \in B_{2}$. It is plain that $p$ is a proximal super-differential of $u$ if and only if there exists a $K>0$ such that $p$ is a $K$-super-differential of $u$.

(A.6) A function $u$ on $M$ is called $K$-semi-concave if it has a $K$-super-differential at each point. It is equivalent to require that, for each $\varphi \in \Phi$, the function

$$
u \circ \varphi(y)-K\|y\|^{2}
$$

is concave on $B_{2}$. As a consequence, if $u$ is $K$-semi-concave and if $p$ is a proximal super-differential of $u$ at $x$, then $p$ is a $K$-super-differential of $u$ at $x$.

(A.7) Let $u$ be a continuous function on $M$, and let Let $A$ be the compact subset of $M$ formed by points $x \in M$ at which both the functions $u$ and $-u$ have a $K$ super-differential. Then the function $u$ is differentiable at each point of $A$, and the mapping $x \longmapsto d u(x)$ is Lipschitz on A, with a Lipschitz constant that depends only on $K$.

This follows from Proposition 4.5.3 in Fathi's book [15. We have the following useful corollary.

(A.8) Let $\mathbb{U}$ be an equi-semi-concave set of functions. Then there exists a constant $K$ with the following property: If $u$ and $v$ are two functions of $\mathbb{U}$ and if $A$ is the set of points minimizing the sum $u+v$, then the functions $u$ and $v$ are differentiable at each point of $A$, and the mapping $A \ni x \longmapsto d u_{x}=-d v_{x}$ is K-Lipschitz.

(A.9) If $u_{n}$ is a sequence of $K$-semi-concave functions converging uniformly to a function $u$, then the function $u$ is $K$-semi-concave. In addition, if $x_{n}$ is a sequence of points of differentiability of $u_{n}$, converging to a point of differentiability $x$ of $u$, then $d u_{n}\left(x_{n}\right) \longrightarrow d u(x)$.

Proof. By the Theorem below, the functions $u_{n}$ form an equi-semi-concave, hence equi-Lipschitz, family of functions. Let $x_{n}$ be a sequence converging to $x$ and $p_{n}$ be a $K$-super-differential of $u_{n}$ at $x_{n}$. The sequence $p_{n}$ is bounded, hence we assume that $p_{n} \longrightarrow p$. Let $y \in B_{2}$ and $\varphi \in \Phi$ be such that $\varphi(y)=x$. For $n$ large enough, the point $x_{n}$ can be written $\varphi\left(y_{n}\right)$ with $y_{n} \in B_{2}$. We have the inequality

$$
u_{n} \circ \varphi(z) \leqslant u_{n} \circ \varphi\left(y_{n}\right)+p_{n} \circ d \varphi_{y_{n}}\left(z-y_{n}\right)+K\left\|z-y_{n}\right\|^{2} \text {, }
$$


for each $z \in B_{2}$, and at the limit, we obtain

$$
u \circ \varphi(z) \leqslant u \circ \varphi(y)+p \circ d \varphi_{y}(z-y)+K\|z-y\|^{2} .
$$

It means that $p$ is a $K$-super-differential of $u$ at $x$. Under the assumptions of the statement, we have $p_{n}=d u_{x_{n}}$, and $p=d u_{x}$ is the only possible limit of this bounded sequence, which is thus converging to $p$.

(A.10) Theorem. A family $\mathbb{U}$ of functions is equi-semi-concave if and only if there exists a number $K>0$ such that all the functions of $\mathbb{U}$ are $K$-semi-concave. In this case, there exists a bounded subset $F \subset C^{2}(M, \mathbb{R})$ and, for each $u \in \mathbb{U}, a$ subset $F_{u}$ of $F$ which has the following properties:

$$
u=\min _{f \in F_{u}} f
$$

and, for each point $x \in M$ and each super-differential $p$ of $u$ at $x$, there exists a function $f \in F_{u}$ such that $(f(x), d f(x))=(u(x), p)$.

In order to prove this result, we need a Lemma:

Lemma. For each $K>0$, there exists a bounded subset $L_{K}$ of $T^{*} M$ which contains all the proximal super-differentials of all $K$-semi-concave functions. As a consequence, the K-semi-concave functions are equi-Lipschitz.

Proof of the Lemma. Let us consider a chart $\varphi \in \Phi$, a $K$-semi-concave function $u$, a point $y_{0} \in B_{1}$, and the point $x_{0}=\varphi\left(y_{0}\right)$. Let $p_{0}$ be a proximal super-differential of $u$ at $x_{0}$, and let us set $l=p_{0} \circ d \varphi_{y_{0}}$.

Claim. If $\|l\| \geqslant 11 K$, then there exists a point $y \in B_{2}$ which is a point of differentiability of $u \circ \varphi$ and satisfies

$$
\left\|d(u \circ \varphi)_{y}\right\| \geqslant(\|l\|-11 K) / 3
$$

and

$$
u \circ \varphi(y)<\inf _{B_{1}} u \circ \varphi
$$

Proof of the Claim. Let us first prove that the infimum of $u \circ \varphi$ in $B_{2}$ is not reached in $\bar{B}_{1}$. Assume, by contradiction, that there exists a point $m \in \bar{B}_{1}$ such that $u \circ \varphi(m)=\inf _{B_{2}} u \circ \varphi$. Then clearly the function $u \circ \varphi$ is differentiable at $m$, its differential is zero, and the inequality

$$
u \circ \varphi\left(y_{0}\right) \leqslant u \circ \varphi(m)+K\left\|y_{0}-m\right\|^{2}
$$

holds. On the other hand, we have

$$
u \circ \varphi(m) \leqslant u \circ \varphi(z) \leqslant u \circ \varphi\left(y_{0}\right)+l\left(z-y_{0}\right)+K\left\|z-y_{0}\right\|^{2}
$$

for all $z \in B_{2}$. Combining these inequalities gives

$$
l\left(y_{0}-z\right) \leqslant K\left\|z-y_{0}\right\|^{2}+K\left\|y_{0}-m\right\|^{2}
$$

for all $z \in \bar{B}_{2}$. Hence $\|l\| \leqslant 5 K$, which is in contradiction with the hypothesis.

Let us now consider a vector $v \in \mathbb{R}^{d}$ of norm 1 and such that $l(v)=-\|l\|$. We get

$$
u \circ \varphi\left(y_{0}+v\right)-u \circ \varphi\left(y_{0}\right) \leqslant l(v)+K\|v\|^{2}=K-\|l\| .
$$


Hence the infimum of $u \circ \varphi$ on $B_{2}$ is not greater than $u \circ \varphi\left(y_{0}\right)+K-\|l\|$. It is then possible to choose a point $y$ in $B_{2}$ such that

$$
u \circ \varphi(y)<\min \left(\inf _{B_{1}} u \circ \varphi, u \circ \varphi\left(y_{0}\right)+2 K-\|l\|\right) .
$$

In addition, since the function $u \circ \varphi$ is differentiable almost everywhere, we can assume that the function $u \circ \varphi$ is differentiable at $y$. We have the inequality

$$
u \circ \varphi\left(y_{0}\right) \leqslant u \circ \varphi(y)+d(u \circ \varphi)_{y}\left(y-y_{0}\right)+K\left\|y-y_{0}\right\|^{2}
$$

from which follows

$$
d(u \circ \varphi)_{y}\left(y_{0}-y\right) \leqslant u \circ \varphi(y)-u \circ \varphi\left(y_{0}\right)+K\left\|y-y_{0}\right\|^{2} \leqslant 11 K-\|l\| .
$$

Hence $\left\|d(u \circ \varphi)_{y}\right\| \geqslant(\|l\|-11 K) / 3$. This ends the proof of the Claim.

In order to continue the proof of the Lemma, we consider the point $y \in B_{2}$ given by the Claim. There exists a chart $\varphi_{1} \in \Phi$ and a point $y_{1} \in B_{1}$ such that $\varphi_{1}\left(y_{1}\right)=\varphi(y)=: x_{1}$. Note that $u \circ \varphi_{1}$ is differentiable at $x_{1}$, and define

$$
l_{1}:=d\left(u \circ \varphi_{1}\right)_{y_{1}}=d(u \circ \varphi)_{y} \circ d\left(\varphi^{-1} \circ \varphi_{1}\right)_{y_{1}} .
$$

There exists a constant $C>1$, which depends only on the atlas $\Phi$, and such that

$$
\left\|l_{1}\right\| \geqslant\left(\left\|l_{0}\right\|-11 K\right) / C \text {. }
$$

If $l_{0}$ is large enough, then we have $\left\|l_{1}\right\| \geqslant 11 K$; hence we can apply the Lemma again, and find a chart $\varphi_{2}$, a point $y_{2} \in B_{2}$, and a linear form $l_{2}$. In addition, we have

$$
u \circ \varphi_{2}\left(y_{2}\right)<\inf _{\varphi\left(B_{1}\right) \cup \varphi_{1}\left(B_{1}\right)} u,
$$

so that the charts $\varphi, \varphi_{1}$, and $\varphi_{2}$ are different. Now if $\left\|l_{0}\right\|$ is sufficiently large, the process can be continued further, and we can build inductively, for $0 \leqslant i \leqslant N$, a sequence $x_{i} \in B_{1}$ of points, a sequence $\varphi_{i} \in \Phi$ of different charts, and a sequence $l_{i}$ of linear forms such that $\left\|l_{i+1}\right\| \geqslant\left(\left\|l_{i}\right\|-11 K\right) / C$. The process can be continued as long as $\left\|l_{i}\right\| \geqslant 11 K$. Recall that the cardinal of $\Phi$ is finite, and denote it by $|\Phi|$. Since all the charts involved in the construction above are different, at most $|\Phi|$ steps can be performed. Hence there exists an integer $N \leqslant|\Phi|$ such that $\left\|l_{i}\right\| \geqslant 11 K$ for $i<N$, and $\left\|l_{N}\right\| \leqslant 11 K$. This gives a bound to $\|l\|$, hence to $\|p\|$.

We now finish the proof of the Theorem. Let us consider a smooth function $g: \mathbb{R}^{d} \longrightarrow \mathbb{R}$ such that $0 \leqslant g \leqslant 1$, and such that $g=0$ outside of $B_{2}$ and $g=1$ inside $B_{1}$. Since the $K$-semi-concave functions are equi-Lipschitz, and since the manifold $M$ is compact, there exists a number $\Delta>0$ such that

$$
\max u-\min u \leqslant \Delta
$$

for each $K$-semi-concave function $u$. Let us associate, to each chart $\varphi \in \Phi$ and each point $(x, p) \in T_{x} M$ satisfying $x \in \varphi\left(B_{1}\right)$, the function $f_{x, p, \varphi}: M \longrightarrow \mathbb{R}$ defined by

$$
f_{x, p, \varphi} \circ \varphi(z):=g(z)\left(p \circ d \varphi_{y}(z-y)+K\|z-y\|^{2}\right)+(1-g(z)) \Delta
$$


for $z \in B_{2}$, where $y=\varphi^{-1}(x)$, and $f_{x, p, \varphi}=\Delta$ outside of $\varphi\left(B_{2}\right)$. The functions $f_{x, p, \varphi},(x, p) \in L_{K}, \varphi \in \Phi$, form a bounded subset $F$ of $C^{2}(M, \mathbb{R})$. For each $K$ semi-concave function $u$, let $F_{u} \subset F+\mathbb{R}$ be the set of functions

$$
z \longmapsto f_{x, p, \varphi}(z)+u(x)
$$

where $p$ is a $K$-super-differential of $u$ at $x$. We claim that $u=\min _{f \in F_{u}} f$. In order to prove this claim, observe that, for each $y \in B_{1}, \varphi \in \Phi$, and $p$ a $K$-superdifferential of $u$ at $x=\varphi(y)$, we have

$$
f_{x, p, \varphi}-u(x) \geqslant u
$$

with equality at $x$. Indeed, we have the inequalities

$$
u \circ \varphi(z)-u(x) \leqslant p \circ d \varphi_{x}(z-y)+K\|z-y\|^{2}
$$

for $z \in B_{2}$ and $u \leqslant u(x)+\Delta$.

THEOREM

\section{B Uniform families of Hamiltonians}

Let us fix once and for all a Riemann metric on the compact manifold $M$. We use this metric to define a norm $|v|$ for tangent vectors and a norm $|p|$ for tangent covectors.

(B.1) A family of pairs $(H, L)$ of dual Hamiltonians and Lagrangians satisfying the hypotheses (1.1) and (1.2) is called uniform if:

(i) There exist two superlinear functions $h_{0}$ and $h_{1}: \mathbb{R}^{+} \longrightarrow \mathbb{R}$ such that each Hamiltonian $H$ of the family satisfies $h_{0}(|p|) \leqslant H(t, x, p) \leqslant h_{1}(|p|)$.

(ii) There exists an increasing function $K(k): \mathbb{R}^{+} \longrightarrow \mathbb{R}^{+}$such that, if $\phi$ is the flow of a Hamiltonian of the family and if the times $t$ and $s$ satisfy $t-1 \leqslant s \leqslant t+1$, then

$$
\phi_{t}^{s}(\{|p| \leqslant k\}) \subset\{|p| \leqslant K(k)\} \subset T^{*} M .
$$

(iii) There exists a finite atlas $\Psi$ of $M$ such that, for each chart $\psi \in \Psi$ and each Lagrangian $L$ of the family, we have $\left\|d^{2}(L \circ T \psi)_{(t, x, v)}\right\| \leqslant K(k)$ for $|v| \leqslant k$.

Note that condition $(i)$ could have equivalently been replaced by the following:

$\left(i^{\prime}\right)$ There exist two superlinear functions $l_{0}$ and $l_{1}: \mathbb{R}^{+} \longrightarrow \mathbb{R}$ such that each Lagrangian $L$ of the family satisfies $l_{0}(|v|) \leqslant L(t, x, v) \leqslant l_{1}(|v|)$.

(B.2) The uniform families of highest use are the following. If $H$ is a Hamiltonian, and if $\omega$ is a bounded finite-dimensional convex family of closed one-forms on $M$, then the Hamiltonians $H\left(t, x, p+\omega_{x}\right), \omega \in \Omega$, form a uniform family. Equivalently, the Lagrangians $L(t, x, v)-\omega_{x}(v)$ form a uniform family.

(B.3) In a uniform family, we have

$$
\left|\partial_{p} H(t, x, p)\right| \geqslant \frac{h_{0}(|p|)-h_{1}(0)}{|p|}
$$

and

$$
\left|\partial_{v} L(t, x, v)\right| \geqslant \frac{l_{0}(|v|)-l_{1}(0)}{|v|} .
$$

In other words, the Legendre transforms are uniformly proper. 
Proof. In view of the convexity of $H$, we have

$$
\left|\partial_{p} H_{(t, x, p)}\right| \geqslant \frac{H(t, x, p)-H(t, x, 0)}{|p|} .
$$

(B.4) Given a Lagrangian $L$ satisfying the hypotheses of (1.2) we define the function $A_{L}(t, x ; s, y): \mathbb{R} \times M \times \mathbb{R} \times M \longrightarrow \mathbb{R}$ by

$$
A_{L}(t, x ; s, y)=\inf _{\gamma \in \Sigma(t, x ; s, y)} \int_{t}^{s} L(\sigma, \gamma(\sigma), \dot{\gamma}(\sigma)) d \sigma,
$$

where $\Sigma(t, x ; s, y)$ is the set of absolutely continuous curves $\gamma:[s, t] \longrightarrow M$ satisfying $\gamma(t)=x$ and $\gamma(s)=y$. We denote by $\Sigma_{m}^{L}(t, x ; s, y)$ the set of curves of $\Sigma(t, x ; s, y)$ which realize the minimum.

(B.5) For each uniform family of Lagrangians, there exists a decreasing function $\left.\left.K_{1}(\epsilon):\right] 0, \infty\right) \longrightarrow \mathbb{R}^{+}$such that, if $L$ is a Lagrangian of the family and if $t$ and $s$ are two real times satisfying $t \geqslant s+\epsilon$, then each curve $\gamma \in \Sigma_{m}^{L}(t, x ; s, y)$ is $C^{2}$ and satisfies $|\dot{\gamma}(\sigma)| \leqslant K(\epsilon)$ for each $\sigma \in[s, t]$.

Proof. Without loss of generality, we can assume that $0<\epsilon<t-s<1$. By comparing the action of $\gamma$ with that of a geodesic with the same endpoints, we get

$$
\int_{s}^{t} l_{0}(|\dot{\gamma}(\sigma)|) d \sigma \leqslant \int_{s}^{t} L(\sigma, \gamma(\sigma), \dot{\gamma}(\sigma)) d \sigma \leqslant(t-s) l_{1}\left(\frac{\operatorname{diam}(M)}{t-s}\right) .
$$

The right hand side is clearly bounded by a constant which depends only of the parameters of the uniform family and of $\epsilon$. We obtain

$$
(t-s) \min l_{0}(|\dot{\gamma}(\sigma)|) \leqslant C,
$$

from which it follows, with another constant $C$, that $\min |\dot{\gamma}(\sigma)| \leqslant C$. But then in view of (B.3), we have

$$
\min _{\sigma \in[s, t]}\left|\partial_{v} L(\sigma, \gamma(\sigma), \dot{\gamma}(\sigma))\right| \leqslant C,
$$

then in view of $(i i)$,

$$
\max _{\sigma \in[s, t]}\left|\partial_{v} L(\sigma, \gamma(\sigma), \dot{\gamma}(\sigma))\right| \leqslant C,
$$

so that finally, using (B.3) again, we get $\max |\dot{\gamma}| \leqslant C$. We have used the symbol $C$ for different constants which depend only of $\epsilon$ and of the parameters of the family.

Note that the proof does not use (iii) in the definition of uniform families.

(B.6) For each times $s<t$, the mapping which, to a Lagrangian L, associates the function

$$
(x, y) \longmapsto A_{L}(s, x, t, y)
$$

of $C(M \times M, \mathbb{R})$, is continuous on each uniform family of Lagrangians endowed with the topology of uniform convergence on compact sets.

Proof. Let $L_{0}$ and $L_{1}$ be two Lagrangians of the family. Let $\gamma(\sigma):[s, t] \longrightarrow M$ be such that

We have

$$
A_{L_{0}}(s, \gamma(s) ; t, \gamma(t))=\int_{s}^{t} L_{0}(\sigma, \gamma(\sigma), \dot{\gamma}(\sigma)) d \sigma .
$$

$$
A_{L_{1}}(s, \gamma(s) ; t, \gamma(t)) \leqslant \int_{s}^{t} L_{1}(\sigma, \gamma(\sigma), \dot{\gamma}(\sigma)) d \sigma
$$


so that

$$
A_{L_{1}}(s, \gamma(s) ; t, \gamma(t))-A_{L_{0}}(s, \gamma(s) ; t, \gamma(t)) \leqslant(t-s) \max _{|v| \leqslant K_{1}(t-s)} L_{1}-L_{0},
$$

where $K_{1}$ is defined in (B.5), By symmetry, we get that

$$
\left\|A_{L_{0}}(s, . ; t, .)-A_{L_{1}}(s, . ; t, .)\right\|_{\infty} \leqslant(t-s) \max _{|v| \leqslant K_{1}(t-s)}\left|L_{1}-L_{0}\right| .
$$

(B.7) Theorem. For each uniform family of Lagrangians and each $\epsilon>0$, consider the set $\mathbb{U}_{\epsilon}$ of continuous functions $M \times M \longrightarrow \mathbb{R}$ given by

$$
(x, y) \longmapsto A_{L}(s, x ; t, y),
$$

where $t \geqslant s+\epsilon$ and $L$ is a Lagrangian of the family. This set is equi-semi-concave, hence equi-Lipschitz on $M \times M$. In addition, for each curve $\gamma \in \Sigma_{m}^{L}(t, x ; s, y)$, the covector

$$
\left(-\partial_{v} L(s, \gamma(s), \dot{\gamma}(s)), \partial_{v} L(t, \gamma(t), \dot{\gamma}(t))\right)
$$

is a proximal super-differential of the function $A_{L}(s, . ; t,$.$) at point (x, y)$.

Proof. Let us consider a finite atlas $\Psi$ of $M$ formed by charts $\psi: B_{6}^{d} \longrightarrow M$, where $B_{r}^{d}$ is the Euclidean ball of radius $r$ in $\mathbb{R}^{d}$. Assume that the open sets $\psi\left(B_{1 / 2}^{d}\right)$, $\psi \in \Psi$, cover $M$. Let $\Phi$ be the atlas of $M \times M$ composed of products $\psi \times \psi^{\prime}$, with $\psi \in \Psi$ and $\psi^{\prime} \in \Psi^{\prime}$. The charts $\varphi$ of $\Phi$ are defined on $B_{3}^{2 d}$, and the images $\varphi\left(B_{1}^{2 d}\right)$, $\varphi \in \Phi$, cover $M \times M$. In order to prove that the set $\mathbb{U}_{\epsilon}$ is equi-semi-concave, we shall check that it is $K$-semi-concave for some $K$. So from now on we shall work in a fixed chart $\varphi=\psi_{0} \times \psi_{1}$.

Let $\left(x_{0}, x_{1}\right)$ be a point in $\psi_{0}\left(B_{2}\right) \times \psi_{1}\left(B_{2}\right)$, and let $y_{0}$ and $y_{1}$ be the preimages in $B_{2}$. Let $\gamma(t):[s, t] \longrightarrow M$ be a curve in $\Sigma_{m}\left(s, x_{0} ; t, x_{1}\right)$. In view of (B.5)] we have $|\dot{\gamma}| \leqslant K_{1}(\epsilon)$. As a consequence, there exists a constant $a>0$, which depends only on the atlas, on the parameters of the family, and on $\epsilon$, such that the curve $\psi_{0}^{-1} \circ \gamma:[s, s+1 / a] \longrightarrow B_{4}^{d}$ is well defined and $a$-Lipschitz, as well as the curve $\psi_{1}^{-1} \circ \gamma:[t-1 / a, t] \longrightarrow B_{4}^{d}$. Let us call $y_{0}(\sigma)$ and $y_{1}(\sigma)$ these curves; note that $y_{0}(s)=y_{0}$ and $y_{1}(t)=y_{1}$. Let us now define, for each points $z_{0}$ and $z_{1}$ in $B_{4}$, the curves

$$
y_{0}\left(\sigma, z_{0}\right):=y_{0}(\sigma)+(1+a(s-\sigma))\left(z_{0}-y_{0}\right)
$$

and

$$
y_{1}\left(\sigma, z_{1}\right):=y_{1}(\sigma)+(1+a(\sigma-t))\left(z_{1}-y_{1}\right) .
$$

For simplicity we define the Lagrangians $L_{0}$ and $L_{1}$ on $\mathbb{R} \times B_{4}^{d} \times \mathbb{R}^{d}$ by the expression $L_{i}(\sigma, x, v)=L\left(\sigma, \psi_{i}(x), d \psi_{i x}(v)\right)$; shortly, $L_{i}=L \circ T \psi_{i}$. We have

$$
\begin{gathered}
A\left(s, \psi_{0}\left(z_{0}\right) ; t, \psi_{1}\left(z_{1}\right)\right) \\
\leqslant A\left(s, x_{0} ; t, x_{1}\right)+\int_{s}^{s+1 / a} L_{0}\left(\sigma, y_{0}\left(\sigma, z_{0}\right), \dot{y}_{0}\left(\sigma, z_{0}\right)\right)-L_{0}\left(\sigma, y_{0}(\sigma), \dot{y}_{0}(\sigma)\right) d \sigma \\
+\int_{t-1 / a}^{t} L_{1}\left(\sigma, y_{1}\left(\sigma, z_{1}\right), \dot{y}_{1}\left(\sigma, z_{1}\right)\right)-L_{1}\left(\sigma, y_{1}(\sigma), \dot{y}_{1}(\sigma)\right) d \sigma .
\end{gathered}
$$

There exists a constant $C>0$, which depends only on the atlas, on the parameters of the family, of $\epsilon$, and of $a$, such that, for $(t, x, v) \in \mathbb{R} \times B_{4}^{d} \times B_{a}^{d}$ and $(y, w) \in$ 
$\mathbb{R} \times B_{4}^{d} \times B_{a}^{d}$, we have

$$
\begin{aligned}
L_{i}(\sigma, y, w)-L_{i}(\sigma, x, v) \leqslant & \partial_{x} L_{i(\sigma, x, v)}(y-x)+\partial_{v} L_{i(\sigma, x, v)}(w-v) \\
& +C\left(\|y-x\|^{2}+\|w-v\|^{2}\right) .
\end{aligned}
$$

We get

$$
\begin{aligned}
A\left(s, \psi_{0}\left(z_{0}\right) ; t, \psi_{1}\left(z_{1}\right)\right) \leqslant & A\left(s, x_{0} ; t, x_{1}\right) \\
+ & \int_{s}^{s+1 / a} \partial_{x} L_{0\left(\sigma, y_{0}(\sigma), \dot{y}_{0}(\sigma)\right)}\left(y_{0}\left(\sigma, z_{0}\right)-y_{0}(\sigma)\right) \\
& +\partial_{v} L_{0\left(\sigma, y_{0}(\sigma), \dot{y}_{0}(\sigma)\right)}\left(\dot{y}_{0}\left(\sigma, z_{0}\right)-\dot{y}_{0}(\sigma)\right) d \sigma \\
+ & \int_{t-1 / a}^{t} \partial_{x} L_{0\left(\sigma, y_{0}(\sigma), \dot{y}_{0}(\sigma)\right)}\left(y_{0}\left(\sigma, z_{0}\right)-y_{0}(\sigma)\right) \\
& +\partial_{v} L_{0\left(\sigma, y_{0}(\sigma), \dot{y}_{0}(\sigma)\right)}\left(\dot{y}_{0}\left(\sigma, z_{0}\right)-\dot{y}_{0}(\sigma)\right) d \sigma \\
+ & C \int_{s}^{s+1 / a}\left\|y_{0}(\sigma)-y_{0}\left(\sigma, z_{0}\right)\right\|^{2}+\left\|\dot{y}_{0}(\sigma)-\dot{y}_{0}\left(\sigma, z_{0}\right)\right\|^{2} d \sigma \\
+ & C \int_{t-1 / a}^{t}\left\|y_{1}(\sigma)-y_{1}\left(\sigma, z_{1}\right)\right\|^{2}+\left\|\dot{y}_{1}(\sigma)-\dot{y}_{1}\left(\sigma, z_{1}\right)\right\|^{2} d \sigma .
\end{aligned}
$$

Taking advantage of the Euler-Lagrange equations, this simplifies to

$$
\begin{aligned}
& A\left(s, \psi_{0}\left(z_{0}\right) ; t, \psi_{1}\left(z_{1}\right)\right) \leqslant A\left(s, x_{0} ; t, x_{1}\right)-\partial_{v} L_{0\left(\sigma, y_{0}, \dot{y}_{0}(s)\right)}\left(z_{0}-y_{0}\right) \\
& +\partial_{v} L_{1\left(\sigma, y_{1}, \dot{y}_{1}(t)\right)}\left(z_{1}-y_{1}\right)+C \frac{1+a^{2}}{a}\left(\left\|y_{0}-z_{0}\right\|^{2}+\left\|y_{1}-z_{1}\right\|^{2}\right) .
\end{aligned}
$$

\section{ACKNOWLEDGEMENTS}

I thank Marie-Claude Arnaud, Alain Chenciner and Albert Fathi for their comments which helped me to improve the present text.

\section{REFERENCES}

[1] V. I. ARnold, Instability of dynamical systems with several degrees of freedom. Sov. Math. Doklady 5 (1964), 581-585. MR0163026(29:329)

[2] P. Bernard, Homoclinic orbits to invariant sets of quasi-integrable exact maps. Ergodic Theory Dynam. Systems 20 (2000), no. 6, 1583-1601. MR.1804946 (2001m:37129)

[3] P. Bernard, Connecting orbits of time dependent Lagrangian systems. Ann. Inst. Fourier 52 (2002), 1533-1568. MR.1935556 (2003m:37088)

[4] P. Bernard, The action spectrum near positive definite invariant tori. Bull. Soc. Math. France 131 (2003), no. 4, 603-616. MR2044497 (2004m:37111)

[5] P. Bernard, G. Contreras, Generic properties of families of Lagrangian systems. Ann. of Math., to appear.

[6] M. Berti, L. Biasco, P. Bolle, Drift in phase space: a new variational mechanism with optimal diffusion time. J. Math. Pures Appl. (9) 82 (2003), no. 6, 613-664. MR 1996776 (2005h:37130)

[7] U. BESSI, An approach to Arnold's diffusion through the calculus of variations. Nonlinear Anal. 26 (1996), no. 6, 1115-1135. MR1375654(97b:58123)

[8] S. Bolotin, D. Treschev, Unbounded growth of energy in nonautonomous Hamiltonian systems. Nonlinearity 12 (1999), no. 2, 365-388. MR1677779 (99m:58086)

[9] C.-Q. Cheng, J. Yan, Existence of diffusion orbits in a priori unstable Hamiltonian systems. J. Differential Geom. 67 (2004), no. 3, 457-517. MR2153027 (2006d:37110)

[10] C.-Q. Cheng, J. Yan, Arnold diffusion in Hamiltonian systems: the a priori unstable case, préprint. 
[11] G. Contreras, G. P. Paternain, Connecting orbits between static classes for generic Lagrangian systems. Topology 41 (2002), no. 4, 645-666. MR.1905833 (2003i:37059)

[12] G. Contreras, J. Delgado, R. Iturriaga, Lagrangian flows: the dynamics of globally minimizing orbits. II. Bol. Soc. Brasil. Mat. (N.S.) 28 (1997), no. 2, 155-196. MR1479500 (98i:58093)

[13] A. Delshams, R. de la Llave, T. Seara, A geometric mechanism for diffusion in Hamiltonian systems overcoming the large gap problem: announcement of results. Electron. Res. Announc. Amer. Math. Soc. 9 (2003), 125-134 (electronic). MR2029474 (2004j:37118)

[14] W. E, Aubry-Mather theory and periodic solutions of the forced Burgers equation. Commun. Pure Appl. Math. 52 (1999), no. 7, 811-828. MR1682812 (2000b:37068)

[15] A. FATHI, Book in preparation.

[16] A. FAтHI, Théorème KAM faible et théorie de Mather sur les systèmes lagrangiens. (French) [A weak KAM theorem and Mather's theory of Lagrangian systems]. C. R. Acad. Sci. Paris Ser. I Math. 324 (1997), no. 9, 1043-1046. MR1451248 (98g:58151)

[17] A. FAthi, Solutions KAM faibles conjuguées et barrières de Peierls. (French) [Weakly conjugate KAM solutions and Peierls's barriers]. C. R. Acad. Sci. Paris Ser. I Math. 325 (1997), no. 6, 649-652. MR 1473840 (99b:58209)

[18] A. Fathi, Orbites hétéroclines et ensemble de Peierls. (French) [Heteroclinic orbits and the Peierls set]. C. R. Acad. Sci. Paris Ser. I Math. 326 (1998), no. 10, 1213-1216. MR1650195 (99k:58066)

[19] V. Kaloshin, Geometric proofs of Mather's connecting and accelerating theorems, Topics in dynamics and ergodic theory. London Math. Soc. Lecture Note Ser. 310 (2003), 81-106, Cambridge Univ. Press, Cambridge. MR2052276 (2005c:37114)

[20] Y. Katznelson, D. Ornstein, Twist maps and Aubry-Mather sets. Lipa's legacy. Contemp. Math., Amer. Math. Soc., Providence, RI, 211 (1997), 343-357. MR1476996 (98j:58067)

[21] P. LOCHAK, Arnold diffusion; a compendium of remarks and questions, in Hamiltonian systems with three or more degrees of freedom. Kluwer Acad. Publ., Dordrecht, 1999, 168183. MR.1720892 (2001g:37107)

[22] J. P. MARCo, D. SAuzin, Stability and instability for Gevrey quasi-convex near-integrable Hamiltonian systems. Publ. Math. I. H. E. S. 96 (2002), 199-275 (2003). MR1986314 (2004m:37112)

[23] J. N. MATHER, Action minimizing invariant measures for positive definite Lagrangian systems. Math. Z. 207 (1991), 169-207. MR 1109661 (92m:58048)

[24] J. N. Mather, Variational construction of connecting orbits. Ann. Inst. Fourier 43 (1993), 1349-1368. MR 1275203 (95c:58075)

[25] J. N. MatheR, Variational construction of trajectories for time-periodic Lagrangian systems on the two torus, unpublished manuscript.

[26] J. N. Mather, Arnold diffusion I: Announcement of results. J. Math. Sci. 124, no. 5 (2004), 5275-5289. MR2129140 (2005m:37142)

[27] R. MAÑ́, Lagrangian flows: The dynamics of globally minimizing orbits. Bol. Soc. Bras. Mat. 28 (1997), 141-153. MR.1479499 (98i:58092)

[28] K. F. SiburG, The principle of least action in geometry and dynamics. Lecture Notes in Mathematics, 1844, Springer-Verlag, Berlin, 2004. MR2076302 (2005m:37151)

[29] D. Treschev, Evolution of slow variables in a priori unstable Hamiltonian systems. Nonlinearity 17 (2004), no. 5, 1803-1841. MR2086152(2005g:37116)

[30] Z. XIA, Arnold diffusion: a variational construction, in Proceedings of the International Congress of Mathematicians, Vol. II (Berlin, 1998), Extra Vol. II, 867-877 (electronic), (1998). MR1648133 (99g:58112)

[31] Z. XIA, Arnold Diffusion and instabilities in Hamiltonian dynamics, preprint.

Université Paris-Dauphine, Ceremade, UMr CNRS 7534, Place Marechal Lattre Tassigny, 75775 Paris, Cedex 16, France

E-mail address: patrick.bernard@ceremade.dauphine.fr 